\title{
Stabilization of exact nonlinear Timoshenko Beams in Space by Boundary Feedback
}

\author{
K. D. Do \\ Department of Mechanical Engineering, Curtin University \\ Bentley, WA 6102, Australia
}

\begin{abstract}
Boundary feedback controllers are designed to stabilize Timoshenko beams with large translational and rotational motions in space under external disturbances. The exact nonlinear partial differential equations governing motion of the beams are derived and used in the control design. The designed controllers guarantee globally practically asymptotically (and locally practically exponentially) stability of the beam motions at the reference state. The control design, well-posedness and stability analysis are based on various relationships between the earth-fixed and body-fixed coordinates, Sobolev embeddings, and a Lyapunov-type theorem developed to study well-posedness and stability for a class of evolution systems in Hilbert space. Simulation results are included to illustrate the effectiveness of the proposed control design.
\end{abstract}

Keywords: Timoshenko beams; Three dimensions; Large motions; Boundary control; Hilbert space; Evolution system.

\section{Introduction}

Timoshenko beams are widely used in practical structures such as poles, bars, columns, and robot arms. In modeling and control of Timoshenko beams, shear deformation and rotational bending effects need to be considered as opposed to neglection of shearing in Bernoulli beams. These effects result in nonlinear couplings between translational and rotational motions of the beams. Usually, the beam's motions are already stable but exhibit an unacceptably slow rate of decay. It is therefore of practical interest to introduce boundary controls that increase the margin of stability. Control of Timoshenko beams is different from that of (Bernoulli) slender beams due to the fact that slender beams are usually supported by tension, which providing structural stiffness, in addition to boundary control forces and moments.

There are excellent works on boundary control on small (vibration) motions of Timoshenko beams, where the classical Timoshenko beam model [1] was used, (e.g., [2-10] based on Lyapunov's direct method or [11, 12] based on the backstepping method [13]). Since the model in [1] is obtained by linearizing exact nonlinear partial differential equations (PDEs) governing motions of shear beams, the results of the above works are only valid in the neighborhood of the origin, see also Remark 2.1. In practice, Timoshenko beams work in a wide range of operations, under which the beams deform with a large magnitude of both translational and rotational motions. It is therefore necessary to consider Timoshenko beams using exact nonlinear PDEs in three-dimensional space. Recently, an exact nonlinear model of Timoshenko beams, of which motions are restricted in two-dimensional space, and their boundary control design are addressed in [14]. Related works include (e.g., [1526]) on boundary control of Bernoulli-type beams with small motions; (e.g., [27-31]) on boundary control of slender beams (i.e., the shear magnitude is smaller than that of the spatial gradient of the

Email address: duc@curtin.edu.au (K. D. Do) 
transverse displacements) with large motions. Modelling, boundary control, and stability analysis of Timoshenko beams governed by exact nonlinear PDEs in space have not been considered.

The above discussion motivates the writing of this paper on modelling, boundary control design, and stability analysis of Timoshenko beams in space under external loads. One end of the beam is connected to an actuation system while the other end carries a payload. The new contributions of this paper are highlighted as follows:

- First, exact nonlinear PDEs governing motions of the beam are derived using deformation theory and Newton's law. The unit quaternion is also used for attitude representation of the beams to resolve singularities caused by Euler angles.

- Second, boundary feedback controllers are designed for global practical asymptotical (and local practical exponential) stabilization of the beams based on the Lyapunov direct method. In the control design, a new Lyapunov functional, various Young's and Hölder's inequalities and Sobolev embedding, a nontrivial combination of Earth-fixed and body-fixed coordinates, and cross vector products are used. Moreover, a special intention is made during the control design so that the development can be extended to spinning beams in future.

- Third, well-posedness and stability analysis of the variational solution of the closed-loop system are rigorously studied based on a Lyapunov-type theorem developed for study of well-posedness and stability analysis for a class of nonlinear evolution systems in Hilbert space. The variational solution (instead of the classical solution) is considered because initial conditions and boundary conditions, which include the boundary controls, are allowed to be general (smooth or nonsmooth), see [32] for discussion of the variational solution in depth. This allowance makes the variational solution more practical than the classical solution, which requires very specific (such as smooth) initial conditions and specific (such as natural/compatible) boundary conditions [32].

Notations. The symbols $\wedge$ and $\vee$ denote the infimum and supremum operators, respectively. These operators are also applied to more than two arguments. The symbol col denotes the column operator. The symbol $\times$ denotes the vector cross product operator.

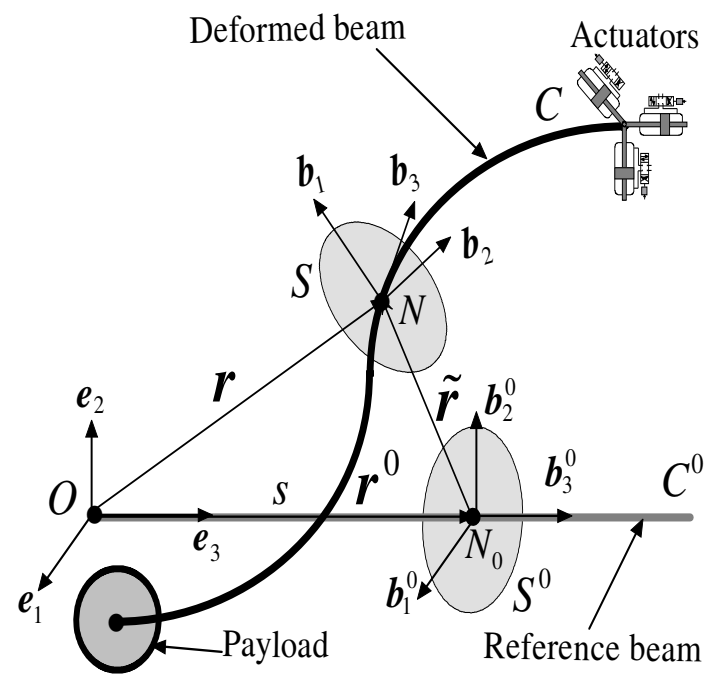

(a) Deformation geometry of the beam.

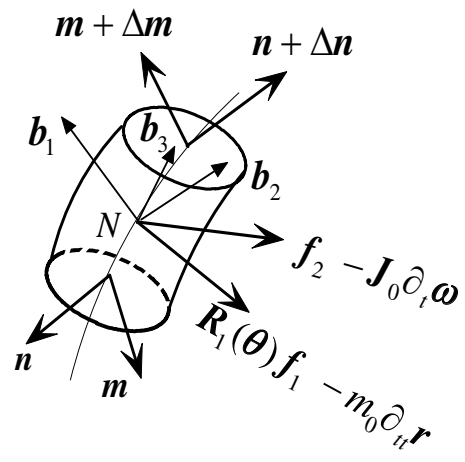

(b) Forces and moments acting on a beam element.

Figure 1: Beam deformation geometry and loading diagram on a beam element.

\section{Mathematical model}

A beam in space as shown in Fig. 1 is considered in this paper. One end of the beam is connected to a payload via a fixed joint while the other end is connected to actuators, which provide boundary 
control forces and moments. The (three) boundary control forces are provided by three linear actuators while the (three) boundary control moments are provided by three rotary actuators. These actuators, which can be electric or hydraulic motors, are installed on a mechanism such that they are completely decouple. An example of a mechanism that decouples all six motions is a 3D guide tube. We assume that plane sections are rigid; and the beam material is (nonlinear) elastic, homogeneous and isotropic. In what follows, equations of motion are briefly derived, see [30] for details.

\subsection{Kinematics}

The reference configuration $\mathcal{B}^{0}$ of the beam in space is described by the position of the base straight line $C^{0}$ parameterized by its arclength coordinate $s$ and the fixed basis $\left(\boldsymbol{b}_{1}^{0}, \boldsymbol{b}_{2}^{0}, \boldsymbol{b}_{3}^{0}\right)$, where $\left(\boldsymbol{b}_{1}^{0}, \boldsymbol{b}_{2}^{0}\right)$ are collinear with the principal axes of inertia of the cross section $S^{0}(s)$ through the base point $N^{0}$, see Fig. 1a. The triple $\left(\boldsymbol{e}_{1}, \boldsymbol{e}_{2}, \boldsymbol{e}_{3}\right)$ is paralleled to $\left(\boldsymbol{b}_{1}^{0}, \boldsymbol{b}_{2}^{0}, \boldsymbol{b}_{3}^{0}\right)$. Thus, $C^{0}$ is described by the position vector $\boldsymbol{r}^{0}(s)$, which in the fixed basis is expressed as $\boldsymbol{r}^{0}(s)=0 \boldsymbol{e}_{1}+0 \boldsymbol{e}_{2}+s \boldsymbol{e}_{3}$. We denote by $\Gamma$ the beam length in its reference state.

The actual configuration $\mathcal{B}$ of the curved beam is described by the actual position $C(s, t)$ of the base curve and the actual configuration $S(s, t)$ of cross sections through the base point $N$. The base curve is described by the position vector $\boldsymbol{r}(s, t)$ while the material cross section is described by the unit vectors $\left\{\boldsymbol{b}_{1}(s, t), \boldsymbol{b}_{2}(s, t), \boldsymbol{b}_{3}(s, t)\right\}$ with $\boldsymbol{b}_{3}$ being aligned with $\boldsymbol{r}_{s}(s, t)$ and $\boldsymbol{b}_{3}=\boldsymbol{b}_{1} \times \boldsymbol{b}_{2}$.

The deformation from $\mathcal{B}^{0}$ to $\mathcal{B}$ is achieved by means of the vector $\boldsymbol{r}(s, t)$ expressed in the local basis, i.e., $\boldsymbol{r}(s, t)=r_{1}(s, t) \boldsymbol{b}_{1}+r_{2}(s, t) \boldsymbol{b}_{2}+r_{3}(s, t) \boldsymbol{b}_{3}$, and the orthogonal tensor $\boldsymbol{R}_{1}(\boldsymbol{\theta}(s, t))$ describing the incremental rigid rotation suffered by $S^{0}(s)$ so that $\boldsymbol{b}_{k}(s, t)=\boldsymbol{R}_{1}(\boldsymbol{\theta}(s, t)) \boldsymbol{b}_{k}^{0}(s), k=1,2,3$ via the sequence $\theta_{1} \rightarrow \theta_{2} \rightarrow \theta_{3}$. The matrix $\boldsymbol{R}_{1}(\boldsymbol{\theta}(s, t))$ is given in components by:

$$
\boldsymbol{R}_{1}(\boldsymbol{\theta})=\left[\begin{array}{ccc}
c_{\theta_{2}} c_{\theta_{3}} & -c_{\theta_{2}} s_{\theta_{3}} & s_{\theta_{2}} \\
c_{\theta_{3}} s_{\theta_{1}} s_{\theta_{2}}+c_{\theta_{1}} s_{\theta_{3}} & c_{\theta_{1}} c_{\theta_{3}}-s_{\theta_{1}} s_{\theta_{2}} s_{\theta_{3}} & -c_{\theta_{2}} s_{\theta_{1}} \\
s_{\theta_{1}} s_{\theta_{3}}-c_{\theta_{1}} c_{\theta_{3}} s_{\theta_{2}} & c_{\theta_{3}} s_{\theta_{1}}+c_{\theta_{1}} s_{\theta_{2}} s_{\theta_{3}} & c_{\theta_{1}} c_{\theta_{2}}
\end{array}\right],
$$

where $\boldsymbol{\theta}:=\operatorname{col}\left(\theta_{1}, \theta_{2}, \theta_{3}\right) ; c_{\theta_{i}}:=\cos \left(\theta_{i}\right)$ and $s_{\theta_{i}}:=\sin \left(\theta_{i}\right)$. This gives $\boldsymbol{b}_{k s}=\boldsymbol{R}_{1 s} \boldsymbol{b}_{k}^{0}$, where $\boldsymbol{R}_{1 s}=\boldsymbol{\mu} \times \boldsymbol{b}_{k}$ with $\boldsymbol{\mu}$ being the axial vector of $\boldsymbol{R}_{1 s} \boldsymbol{R}_{1}^{T}$. The generalized strains (i.e., the stretch $\varepsilon$ and the shear strains $\eta_{1}$ and $\left.\eta_{2}\right)$ are expressed by the stretch vector $\boldsymbol{v}=\eta_{1} \boldsymbol{b}_{1}+\eta_{2} \boldsymbol{b}_{2}+(1+\varepsilon) \boldsymbol{b}_{3}$ in its local basis: $\boldsymbol{v}=\boldsymbol{r}_{s}$. Thus, we have

$$
\begin{aligned}
& \boldsymbol{r}_{s}=\eta_{1} \boldsymbol{b}_{1}+\eta_{2} \boldsymbol{b}_{2}+(1+\varepsilon) \boldsymbol{b}_{3} \\
& \boldsymbol{\mu}=\mu_{1} \boldsymbol{b}_{1}+\mu_{2} \boldsymbol{b}_{2}+\mu_{3} \boldsymbol{b}_{3}, \omega=\omega_{1} \boldsymbol{b}_{1}+\omega_{2} \boldsymbol{b}_{2}+\omega_{3} \boldsymbol{b}_{3}, \\
& \boldsymbol{b}_{k s}=\boldsymbol{\mu} \times \boldsymbol{b}_{k}, \boldsymbol{b}_{k t}=\omega \times \boldsymbol{b}_{k},\left(\boldsymbol{\mu} \times \boldsymbol{b}_{k}\right)_{t}=\left(\boldsymbol{\omega} \times \boldsymbol{b}_{k}\right)_{s} .
\end{aligned}
$$

From (1) and (2) we have

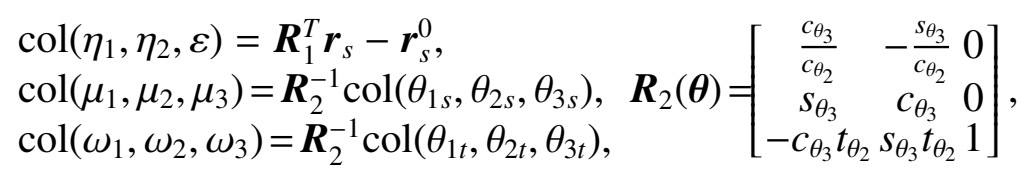

where $t_{\theta_{2}}:=\tan \left(\theta_{2}\right)$.

\subsection{Kinetic}

Balancing linear and angular momentum on a beam element, see Fig.1b, gives the equations of motion:

$$
\begin{aligned}
& m_{0} \tilde{\boldsymbol{r}}_{t t}=\boldsymbol{n}_{s}+\boldsymbol{R}_{1}(\boldsymbol{\theta}) \boldsymbol{f}_{1}, \\
& \boldsymbol{J}_{0} \boldsymbol{\omega}_{t}=\boldsymbol{m}_{s}+\boldsymbol{r}_{s} \times \boldsymbol{n}-\boldsymbol{\omega} \times \boldsymbol{J}_{0} \boldsymbol{\omega}+\boldsymbol{f}_{2},
\end{aligned}
$$

where $m_{0}$ is the beam mass per unit length; $\boldsymbol{J}_{0}$ is the mass moment matrix of inertia; $\boldsymbol{n}$ and $\boldsymbol{m}$ denote the contact force and moment vectors; and (see Fig. 1a)

$$
\tilde{\boldsymbol{r}}=\boldsymbol{r}-\boldsymbol{r}^{0} .
$$


The nonconservative force and moment vectors $f_{1}$ and $f_{2}$ are given in the body-fixed frame as

$$
\begin{aligned}
& f_{1}=-D_{11} v-D_{12}(v \otimes v) v+f_{10}(t), \\
& f_{2}=-D_{21} \omega-D_{22}(\omega \otimes \omega) \omega+f_{20}(t),
\end{aligned}
$$

where $\boldsymbol{D}_{i j},(i, j)=1,2$ are diagonal and positive definite matrices; $\boldsymbol{a} \otimes \boldsymbol{a}:=\operatorname{diag}\left(a_{1}^{2}, a_{2}^{2}, a_{3}^{2}\right)$ with $\boldsymbol{a}=\operatorname{col}\left(a_{1}, a_{2}, a_{3}\right)$; and $\boldsymbol{f}_{10}(t)$ and $\boldsymbol{f}_{20}(t)$ are external disturbances bounded in $L^{2}$-norm, and $\boldsymbol{v}$ is the linear velocity vector with coordinates in the body-fixed frame, i.e.,

$$
\boldsymbol{v}=\boldsymbol{R}_{1}^{-1}(\boldsymbol{\theta}) \tilde{\boldsymbol{r}}_{t} .
$$

When $\theta_{2}= \pm \frac{\pi}{2}$, there are singularities in (3). Thus, we use the unit quaternion vector $\boldsymbol{q}=\operatorname{col}\left(q_{1}, q_{2}, q_{3}, q_{4}\right)$ for attitude representation with $\|\boldsymbol{q}\|^{2}=1$ relating to $\left(\theta_{1}, \theta_{2}, \theta_{3}\right)$ via the sequence $\theta_{1} \rightarrow \theta_{2} \rightarrow \theta_{3}$ as follows [33]:

$$
\boldsymbol{q}(\boldsymbol{\theta})=\left[\begin{array}{l}
\cos \left(\frac{\theta_{1}}{2}\right) \cos \left(\frac{\theta_{2}}{2}\right) \cos \left(\frac{\theta_{3}}{2}\right)-\sin \left(\frac{\theta_{1}}{2}\right) \sin \left(\frac{\theta_{2}}{2}\right) \sin \left(\frac{\theta_{3}}{2}\right) \\
\sin \left(\frac{\theta_{1}}{2}\right) \cos \left(\frac{\theta_{2}}{2}\right) \cos \left(\frac{\theta_{3}}{2}\right)+\cos \left(\frac{\theta_{1}}{2}\right) \sin \left(\frac{\theta_{2}}{2}\right) \sin \left(\frac{\theta_{3}}{2}\right) \\
\cos \left(\frac{\theta_{1}}{2}\right) \sin \left(\frac{\theta_{2}}{2}\right) \cos \left(\frac{\theta_{3}}{2}\right)-\sin \left(\frac{\theta_{1}}{2}\right) \cos \left(\frac{\theta_{2}}{2}\right) \sin \left(\frac{\theta_{3}}{2}\right) \\
\cos \left(\frac{\theta_{1}}{2}\right) \cos \left(\frac{\theta_{2}}{2}\right) \sin \left(\frac{\theta_{3}}{2}\right)+\sin \left(\frac{\theta_{1}}{2}\right) \sin \left(\frac{\theta_{2}}{2}\right) \cos \left(\frac{\theta_{3}}{2}\right)
\end{array}\right] .
$$

The rotational matrix $\boldsymbol{R}_{1}$ is given in terms of $\boldsymbol{q}$ as follows:

$$
\boldsymbol{R}_{1}(\boldsymbol{q})=\boldsymbol{I}_{3}+2 q_{1} \boldsymbol{S}(\overline{\boldsymbol{q}})+2 \boldsymbol{S}^{2}(\overline{\boldsymbol{q}})
$$

where $\overline{\boldsymbol{q}}:=\operatorname{col}\left(q_{2}, q_{3}, q_{4}\right)$ and the matrix $\boldsymbol{S}(\boldsymbol{x})$ is defined as $\boldsymbol{S}(\boldsymbol{x}) \boldsymbol{y}=\boldsymbol{x} \times \boldsymbol{y}$ for all $(\boldsymbol{x}, \boldsymbol{y}) \in \mathbb{R}^{3}$. Let us also define the matrix:

$$
\boldsymbol{K}(\boldsymbol{q})=\frac{1}{2}\left[\begin{array}{l}
-\overline{\boldsymbol{q}}^{T} \\
q_{1} \boldsymbol{I}_{3}+S(\overline{\boldsymbol{q}})
\end{array}\right] .
$$

With (3), (7), (9), and (10), we can write (4) as the following system of PDEs:

$$
\begin{aligned}
& \tilde{\boldsymbol{r}}_{t}=\boldsymbol{R}_{1}(\boldsymbol{q}) \boldsymbol{v}, \\
& \boldsymbol{q}_{t}=\boldsymbol{K}(\boldsymbol{q}) \boldsymbol{\omega}, \\
& m_{0} \boldsymbol{v}_{t}=m_{0}\left(\boldsymbol{R}_{1}^{-1}(\boldsymbol{q})\right)_{t} \boldsymbol{R}_{1}(\boldsymbol{q}) \boldsymbol{v}+\boldsymbol{R}_{1}^{-1}(\boldsymbol{q}) \boldsymbol{n}_{s}+\boldsymbol{f}_{1}, \\
& \boldsymbol{J}_{0} \boldsymbol{\omega}_{t}=\boldsymbol{m}_{s}-\boldsymbol{\omega} \times\left(\boldsymbol{J}_{0} \boldsymbol{\omega}\right)+\boldsymbol{r}_{s} \times \boldsymbol{n}+\boldsymbol{f}_{2} .
\end{aligned}
$$

The contact force and moment vectors $\boldsymbol{n}$ and $\boldsymbol{m}$ are given by:

$$
\begin{aligned}
& \boldsymbol{n}(s, t)=Q_{1}(s, t) \boldsymbol{b}_{1}+Q_{2}(s, t) \boldsymbol{b}_{2}+N(s, t) \boldsymbol{b}_{3}, \\
& \boldsymbol{m}(s, t)=M_{1}(s, t) \boldsymbol{b}_{1}+M_{2}(s, t) \boldsymbol{b}_{2}+T(s, t) \boldsymbol{b}_{3},
\end{aligned}
$$

where $Q_{1}$ and $Q_{2}$ are the shear forces; $N$ is the axial force; $M_{1}$ and $M_{2}$ are the bending moments; and $T$ is the twisting moment. Using the third-order Maclaurin series expansion of nonlinear stress-strain relations in [34] results in the constitutive equations:

$$
\begin{aligned}
& Q_{i}=G \bar{A}_{i}\left(\eta_{i}-\frac{1}{2} \eta_{i}^{2}+\frac{1}{3} \eta_{i}^{3}\right), N=E A\left(\varepsilon-\frac{1}{2} \varepsilon^{2}+\frac{1}{3} \varepsilon^{3}\right), \\
& M_{i}=E I_{i}\left(\mu_{i}-\frac{1}{2} \mu_{i}^{2}+\frac{1}{3} \mu_{i}^{3}\right), T=G I_{3}\left(\mu_{3}-\frac{1}{2} \mu_{3}^{2}+\frac{1}{3} \mu_{3}^{3}\right),
\end{aligned}
$$

where $i=1,2 ; E$ is the Young modulus; $G$ is the shear modulus; $A, \bar{A}_{1}, \bar{A}_{2}$ are cross section and shear areas; $I_{k}, k=1,2,3$ are principal mass moments of inertia about $\boldsymbol{b}_{k}$.

The initial conditions are given by

$$
\begin{array}{ll}
\tilde{\boldsymbol{r}}\left(s, t_{0}\right)=\tilde{\boldsymbol{r}}_{10}(s), & \tilde{\boldsymbol{r}}_{t}\left(s, t_{0}\right)=\tilde{\boldsymbol{r}}_{20}(s), \\
\boldsymbol{\theta}\left(s, t_{0}\right)=\boldsymbol{\theta}_{0}(s), & \boldsymbol{\omega}\left(s, t_{0}\right)=\boldsymbol{\omega}_{0}(s) .
\end{array}
$$

Finally, referring to Fig. 1 the boundary conditions are given by

$$
\text { At } s=0:\left\{\begin{array}{l}
m_{P} \ddot{\tilde{\boldsymbol{r}}}=\boldsymbol{n}+\boldsymbol{f}_{1}^{B 0}, \\
\dot{\boldsymbol{q}}=\boldsymbol{K}(\boldsymbol{q}) \boldsymbol{\omega}, \\
\boldsymbol{J}_{P} \dot{\boldsymbol{\omega}}=\boldsymbol{m}+\boldsymbol{f}_{2}^{B 0},
\end{array} \quad \text { At } s=\Gamma:\left\{\begin{array}{l}
\boldsymbol{M}_{H} \ddot{\tilde{\boldsymbol{r}}}=-\boldsymbol{n}+\boldsymbol{\phi}_{1 B}+\boldsymbol{f}_{1}^{B \Gamma}, \\
\dot{\boldsymbol{q}}=\boldsymbol{K}(\boldsymbol{q}) \boldsymbol{\omega}, \\
\boldsymbol{J}_{H} \dot{\boldsymbol{\omega}}=-\boldsymbol{m}+\boldsymbol{\phi}_{2 B}+\boldsymbol{f}_{2}^{B \Gamma},
\end{array}\right.\right.
$$


where

At $s=0:\left\{\begin{array}{l}\boldsymbol{f}_{1}^{B 0}=-\boldsymbol{D}_{11}^{0} \dot{\tilde{\boldsymbol{r}}}-\boldsymbol{D}_{12}^{0}(\dot{\tilde{\boldsymbol{r}}} \otimes \dot{\tilde{\boldsymbol{r}}}) \dot{\tilde{\boldsymbol{r}}}+\boldsymbol{f}_{10}^{B 0}, \\ \boldsymbol{f}_{2}^{B 0}=-\boldsymbol{D}_{21}^{0} \boldsymbol{\omega}-\boldsymbol{D}_{22}^{0}(\boldsymbol{\omega} \otimes \boldsymbol{\omega}) \boldsymbol{\omega}+\boldsymbol{f}_{20}^{B 0},\end{array} \quad\right.$ At $s=\Gamma:\left\{\begin{array}{l}\boldsymbol{f}_{1}^{B \Gamma}=-\boldsymbol{D}_{11}^{\Gamma} \dot{\tilde{\boldsymbol{r}}}-\boldsymbol{D}_{12}^{\Gamma}(\dot{\tilde{\boldsymbol{r}}} \otimes \dot{\tilde{\boldsymbol{r}}}) \dot{\tilde{\boldsymbol{r}}}+\boldsymbol{f}_{10}^{B \Gamma,} \\ \boldsymbol{f}_{2}^{B \Gamma}=-\boldsymbol{D}_{21}^{\Gamma} \boldsymbol{\omega}-\boldsymbol{D}_{22}^{\Gamma}(\boldsymbol{\omega} \otimes \omega) \boldsymbol{\omega}+\boldsymbol{f}_{20}^{B \Gamma},\end{array}\right.$

and $m_{p}$ and $\boldsymbol{J}_{P}$ are the mass and matrix of inertia moment of the payload; $\boldsymbol{M}_{H}$ and $\boldsymbol{J}_{H}$ are mass and inertia moment matrices of the actuator systems; $\boldsymbol{D}_{i j}^{0}$ and $\boldsymbol{D}_{i j}^{\Gamma}$ are positive definite and diagonal damping matrices; $\phi_{1 B}=\operatorname{col}\left(\phi_{11 B}, \phi_{12 B}, \phi_{13 B}\right)$ and $\phi_{2 B}=\operatorname{col}\left(\phi_{21 B}, \phi_{22 B}, \phi_{23 B}\right)$ are force and moment boundary control input vectors; and $\boldsymbol{f}_{i 0}^{0}$ and $\boldsymbol{f}_{i 0}^{\Gamma}$ are external force and moment vectors acting on the payload and actuators. Note that $\boldsymbol{f}_{10}^{B 0}$ and $\boldsymbol{f}_{10}^{B \Gamma}$ are coordinated in the earth-fixed frame while $\boldsymbol{f}_{20}^{B 0}$ and $f_{20}^{B \Gamma}$ are coordinated in the body-fixed frame.

Remark 2.1. Linearization of (11), (3), and (13) will result in a system modelling the classical Timoshenko beams, which were considered in existing works (e.g., [2-9, 11, 12]). The linearized system excludes couplings of the longitudinal motion with transverse and rotational motions. Moreover, recent work [14] restricts motions of the beam to two dimensional space. From (11) and (3), it is clearly seen that all the translational and rotational motions of the beam are nonlinearly coupled in all dimensions via the matrices $\boldsymbol{R}_{1}(\boldsymbol{q})$ and $\boldsymbol{K}(\boldsymbol{q})$, and the term $\boldsymbol{r}_{s} \times \boldsymbol{n}$, see (11) and (3). Therefore, the existing works $([2-9,11,12,14])$ were not able to address the aforementioned couplings. This paper directly considers the exact nonlinear model comprising (11) and (3). Thus, the aforementioned couplings are considered, and both large and small amplitude translational and rotational motions are controlled. It will be seen in the sequel that due to complex nonlinear couplings between all the motions of the beam in all dimensions, the control design is much more involved than the one for two-dimensional space in [14].

\section{Control objective}

Before stating the control objective, we make the following assumption, which is reasonable in practice, on boundedness of initial values and external loads.

\section{Assumption 3.1.}

1) The initial values $\tilde{\boldsymbol{r}}_{10}(s), \tilde{\boldsymbol{r}}_{20}(s), \boldsymbol{\theta}_{0}(s), \omega_{0}(s)$ are bounded in $L^{2}$-norm, i.e., there exists $\epsilon_{0} \geq 0$ such that $\int_{0}^{\Gamma}\left(\left\|\tilde{\boldsymbol{r}}_{10}(s)\right\|^{2}+\left\|\tilde{\boldsymbol{r}}_{20}(s)\right\|^{2}+\left\|\boldsymbol{\theta}_{0}(s)\right\|^{2}+\left\|\boldsymbol{\omega}_{0}(s)\right\|^{2}\right) d s \leq \epsilon_{0}$. Moreover, $\left\|\tilde{\boldsymbol{r}}_{1}\left(0, t_{0}\right)\right\|^{2}+\left\|\tilde{\boldsymbol{r}}_{2}\left(0, t_{0}\right)\right\|^{2}+$ $\left\|\boldsymbol{\theta}\left(0, t_{0}\right)\right\|^{2}+\left\|\boldsymbol{\omega}\left(0, t_{0}\right)\right\|^{2}$ and $\left\|\tilde{\boldsymbol{r}}\left(\Gamma, t_{0}\right)\right\|^{2}+\left\|\tilde{\boldsymbol{r}}_{t}\left(\Gamma, t_{0}\right)\right\|^{2}+\left\|\boldsymbol{\theta}\left(\Gamma, t_{0}\right)\right\|^{2}+\left\|\boldsymbol{\omega}\left(\Gamma, t_{0}\right)\right\|^{2}$ are also bounded.

2) The external loads are bounded in appropriate norms, i..e, there exist nonnegative constants $f_{i 0}^{M}, f_{i 0}^{B 0 M}$, and $f_{i 0}^{B \Gamma M}$ such that

$$
\sup _{t \in\left[t_{0}, \infty\right)} \int_{0}^{\Gamma}\left\|\boldsymbol{f}_{i 0}(s, t)\right\|^{2} d s \leq f_{i 0}^{M}, \sup _{t \in\left[t_{0}, \infty\right)}\left\|\boldsymbol{f}_{i 0}^{B 0}(t)\right\|^{2} \leq f_{i 0}^{B 0 M}, \sup _{t \in\left[t_{0}, \infty\right)}\left\|\boldsymbol{f}_{i 0}^{B \Gamma}(t)\right\|^{2} \leq f_{i 0}^{B \Gamma M} .
$$

Control Objective 3.1. Under Assumption 3.1, design the boundary control vectors $\phi_{i B}, i=1,2$ such that the beam system consisting of (11)-(15) is globally practically asymptotically (and locally practically exponentially) stable at the origin in the sense that

$$
\mathcal{E}(t) \leq \mathcal{E}\left(t_{0}\right) e^{-c\left(t-t_{0}\right)}+c_{0},
$$

where $c$ is a positive constant depending on the initial data when the data are large, and is independent of the initial data when the data are small, and the constant $c_{0}$ is a nonnegative constant. The function $\mathcal{E}(t)$ is given by

$$
\begin{aligned}
\mathcal{E}(t)= & \int_{0}^{\Gamma}\left[\|\boldsymbol{v}(s, t)\|^{2}+\|\boldsymbol{\omega}(s, t)\|^{2}+\|\boldsymbol{\vartheta}(s, t)\|^{2}+\|\boldsymbol{\mu}(s, t)\|^{2}+\left(\eta_{1}^{4}(s, t)+\eta_{2}^{4}(s, t)+\varepsilon^{4}(s, t)\right)\right. \\
& \left.+\left(\mu_{1}^{4}(s, t)+\mu_{2}^{4}(s, t)+\mu_{3}^{4}(s, t)\right)\right] d s+\mathcal{E}^{B 0}(t)+\mathcal{E}^{B \Gamma}(t), \\
\mathcal{E}^{B 0}(t) & =\|\dot{\tilde{\boldsymbol{r}}}(0, t)\|^{2}+\|\boldsymbol{\omega}(0, t)\|^{2}, \\
\mathcal{E}^{B \Gamma}(t) & =\left\|\gamma_{1} \frac{\tilde{\boldsymbol{r}}(\Gamma, t)}{\Delta(\Gamma, t)}+\dot{\tilde{\boldsymbol{r}}}(\Gamma, t)+\gamma \Gamma \boldsymbol{R}_{1}(\boldsymbol{q}(\Gamma, t)) \boldsymbol{\vartheta}(\Gamma, t)\right\|^{2}+\Delta(\Gamma, t)-1 \\
& +\left\|\gamma_{2} \boldsymbol{K}^{T}(\boldsymbol{q}(\Gamma, t)) \operatorname{col}\left(q_{1}(\Gamma, t)-1, \overline{\boldsymbol{q}}(\Gamma, t)\right)+\omega(\Gamma, t)+\gamma \Gamma \boldsymbol{\mu}(\Gamma, t)\right\|^{2}+\left\|\operatorname{col}\left(q_{1}(\Gamma, t), \overline{\boldsymbol{q}}(\Gamma, t)\right)\right\|^{2},
\end{aligned}
$$


where $\gamma, \gamma_{1}$, and $\gamma_{2}$ are positive constants (to be chosen later), and

$$
\begin{aligned}
& \boldsymbol{\vartheta}(s, t):=\operatorname{col}\left(\eta_{1}(s, t), \eta_{2}(s, t), \varepsilon(s, t)\right), \\
& \Delta(\Gamma, t):=\sqrt{1+(\tilde{\boldsymbol{r}}(\Gamma, t))^{T} \boldsymbol{K}_{1 B} \tilde{\boldsymbol{r}}(\Gamma, t),}
\end{aligned}
$$

where $\boldsymbol{K}_{1 B}$ is a positive definite matrix.

Remark 3.1. It is clear that $\mathcal{E}(t)$ is a positive definite and radially unbounded functional of velocities, stretch, shear strain, bending and torsional curvatures. While this functional penalizes (translational and rotational) displacement and velocity motions of the actuated end, it is only necessary to include translational and rotational velocities at the payload end. Convergence of the translational and rotational displacements at any point of the beam including the payload end are ensured by convergence of $\mathcal{E}(t)$ via Sobolev embedding, see the last five inequalities in the lemma below. By doing so, difficulties in the control design will be relaxed.

Several useful equalities and inequalities, which will be used in the control design and stability analysis, are given in the following lemma.

Lemma 3.1. For all $t \geq t_{0} \geq 0$ and $s \in[0, \Gamma]$, we have:

1) $\omega_{s}=\mu_{1 t} \boldsymbol{b}_{1}+\mu_{2 t} \boldsymbol{b}_{2}+\mu_{3 t} \boldsymbol{b}_{3}$,

2) $\boldsymbol{\omega}^{T}\left(\boldsymbol{r}_{s} \times \boldsymbol{n}\right)=\boldsymbol{n}^{T}\left(\eta_{1} \boldsymbol{b}_{1 t}+\eta_{2} \boldsymbol{b}_{2 t}+(1+\varepsilon) \boldsymbol{b}_{3 t}\right)$,

3) $\boldsymbol{\mu}^{T}\left(\boldsymbol{r}_{s} \times \boldsymbol{n}\right)=-\boldsymbol{n}^{T}\left(\eta_{1} \boldsymbol{b}_{1 s}+\eta_{2} \boldsymbol{b}_{2 s}+(1+\varepsilon) \boldsymbol{b}_{3 s}\right)$,

4) $\boldsymbol{n}^{T} \tilde{\boldsymbol{r}}_{s t}=Q_{1} \eta_{1 t}+Q_{2} \eta_{2 t}+N \varepsilon_{t}+\omega^{T}\left(\boldsymbol{r}_{s} \times n\right)$,

5) $\boldsymbol{n}^{T}\left(\boldsymbol{r}_{s}+\boldsymbol{r}_{s s} s\right)=Q_{1} \eta_{1}+Q_{2} \eta_{2}+N(1+\varepsilon)+Q_{1} \eta_{1 s} s+Q_{2} \eta_{2 s} s+N \varepsilon_{s} s+\boldsymbol{n}^{T}\left(\boldsymbol{\mu} \times \boldsymbol{r}_{s}\right) s$,

6) $\int_{0}^{\Gamma}\|\tilde{\boldsymbol{r}}(s, t)\|^{2} \leq 2 \Gamma\|\tilde{\boldsymbol{r}}(\Gamma, t)\|^{2}+4 \Gamma^{2} \int_{0}^{\Gamma}\left\|\tilde{\boldsymbol{r}}_{s}(s, t)\right\|^{2} d s$,

7) $\|\tilde{\boldsymbol{r}}(0, t)\|^{2} \leq 2\|\tilde{\boldsymbol{r}}(\Gamma, t)\|^{2}+4 \Gamma \int_{0}^{\Gamma}\left\|\tilde{\boldsymbol{r}}_{s}(s, t)\right\|^{2} d s$,

8) $\quad \int_{0}^{\Gamma}\|\tilde{\boldsymbol{r}}(s, t)\|^{2} d s \leq 2 \int_{0}^{\Gamma}\|\boldsymbol{\vartheta}(s, t)\|^{2} d s+1152 \Gamma^{2} \int_{0}^{\Gamma}\|\boldsymbol{\mu}(s, t)\|^{2} d s+96 \Gamma\left(q_{2}^{2}(\Gamma, t)+q_{3}^{2}(\Gamma, t)\right)$,

9) $\quad\left\|\operatorname{col}\left(q_{1}(0, t)-1, \overline{\boldsymbol{q}}(0, t)\right)\right\|^{2} \leq 2\left\|\operatorname{col}\left(q_{1}(\Gamma, t)-1, \overline{\boldsymbol{q}}(\Gamma, t)\right)\right\|^{2}+\Gamma \int_{0}^{\Gamma}\|\boldsymbol{\mu}(s, t)\|^{2} d s$,

10) $\int_{0}^{\Gamma}\left\|\operatorname{col}\left(q_{1}(s, t)-1, \overline{\boldsymbol{q}}(s, t)\right)\right\|^{2} d s \leq 2\left\|\operatorname{col}\left(q_{1}(\Gamma, t)-1, \overline{\boldsymbol{q}}(\Gamma, t)\right)\right\|^{2}+\Gamma^{2} \int_{0}^{\Gamma}\|\boldsymbol{\mu}(s, t)\|^{2} d s$,

where we have dropped the argument $(s, t)$ for clarity. The inequality 8$)$ is of its own interest because the right hand-side depends on $\left(q_{2}^{2}(\Gamma, t)+q_{3}^{2}(\Gamma, t)\right)$ not on $q_{1}(\Gamma, t)$ and $q_{4}(\Gamma, t)$. This makes the boundary control design in this paper applicable to spinning beams such as drillers because the right hand-side of the inequality 8$)$ does not depend on $\theta_{3}(\Gamma, t)$, which is inferred from (8), i.e., $q_{2}^{2}+q_{3}^{2}=\sin ^{2}\left(\frac{\theta_{1}}{2}\right) \cos ^{2}\left(\frac{\theta_{2}}{2}\right)+\cos ^{2}\left(\frac{\theta_{1}}{2}\right) \sin ^{2}\left(\frac{\theta_{2}}{2}\right)$ for all $s \in[0, \Gamma]$ and $t \in\left[t_{0}, \infty\right)$.

Proof. Proof of the first 5 equalities is given in the proof of Lemma 3.1 in [30] while proof of the sixth and seventh inequalities is given in the proof of Lemma 2.1 in [14]. We here provide the proof of the last three inequalities. From (5) and the first equation of (3), we have

$$
\tilde{\boldsymbol{r}}_{s}=\boldsymbol{R}_{1}(\boldsymbol{q}) \boldsymbol{\vartheta}+\boldsymbol{R}_{1}(\boldsymbol{q}) \boldsymbol{e}_{3}-\boldsymbol{e}_{3},
$$

where $\boldsymbol{\vartheta}$ is defined in (20) and $\boldsymbol{e}_{3}:=\operatorname{col}(0,0,1)$. By taking norm-2 both sides of (22) and applying Young's inequality together with the expression of $\boldsymbol{R}_{1}(\boldsymbol{q})$ in (9), we have

$$
\left\|\tilde{\boldsymbol{r}}_{s}\right\|^{2} \leq 2\|\boldsymbol{\vartheta}\|^{2}+48\left(q_{2}^{2}+q_{3}^{2}\right) .
$$

Using the simplified Poincaré inequality, see proof of Lemma 2.1 in [14] and integrating both sides of (23) from 0 to $\Gamma$ give

$$
\begin{aligned}
\int_{0}^{\Gamma}\left\|\tilde{\boldsymbol{r}}_{s}\right\|^{2} d s & \leq 2 \int_{0}^{\Gamma}\|\boldsymbol{\vartheta}\|^{2} d s+48 \int_{0}^{\Gamma}\left(q_{2}^{2}+q_{3}^{2}\right) d s \\
& \leq 2 \int_{0}^{\Gamma}\|\boldsymbol{\vartheta}\|^{2} d s+192 \int_{0}^{\Gamma}\left(q_{2 s}^{2}+q_{3 s}^{2}\right) d s+96 \Gamma\left(q_{2}^{2}(\Gamma, t)+q_{3}^{2}(\Gamma, t)\right) \\
& \leq 2 \int_{0}^{\Gamma}\|\boldsymbol{\vartheta}\|^{2} d s+1152 \Gamma^{2} \int_{0}^{\Gamma}\|\boldsymbol{\mu}\|^{2} d s+96 \Gamma\left(q_{2}^{2}(\Gamma, t)+q_{3}^{2}(\Gamma, t)\right),
\end{aligned}
$$

where we have use $\boldsymbol{q}_{s}=\boldsymbol{K}(\boldsymbol{q}) \boldsymbol{\mu} \Rightarrow\left\|\boldsymbol{q}_{s}\right\|^{2} \leq 6\|\boldsymbol{\mu}\|^{2}$ with $\boldsymbol{K}(\boldsymbol{q})$ given in (10), which completes proof of the inequality 8). Proof of the inequality 9) is similar to that of the inequality 7) with the use of $\left|q_{1}-1 \overline{\boldsymbol{q}}^{T}\right|_{s}^{T}=\boldsymbol{q}_{s}=\boldsymbol{K}(\boldsymbol{q}) \boldsymbol{\mu}$ and $\boldsymbol{K}^{T}(\boldsymbol{q}) \boldsymbol{K}(\boldsymbol{q})=\frac{1}{4}$. The inequality 10) is proved by applying the simplified Poincaré inequality to $\left\|\left|q_{1}-1 \overline{\boldsymbol{q}}^{T}\right|^{T}\right\|^{2}$ and noting that $\boldsymbol{q}_{s}=\boldsymbol{K}(\boldsymbol{q}) \boldsymbol{\mu}$ and $\boldsymbol{K}^{T}(\boldsymbol{q}) \boldsymbol{K}(\boldsymbol{q})=\frac{1}{4}$. 


\section{Well-posedness and Stability of Nonlinear Evolution Systems}

This section presents results on well-posedness (existence, uniqueness, and continuous dependence on initial conditions) and stability of nonlinear evolution systems to be used in control design and stability analysis of the beam system.

\subsection{Space notations}

Let $H$ be a separable Hilbert space identified with its dual $H^{*}$ by the Riesz isomorphism. Let $V$ be a real reflexible Banach space such that $V \subset H$ continuously and densely, and $V^{*}$ be the dual of $V$. From the definitions of $H$ and $V$, we have that the embedding $V \subset H \equiv H^{*} \subset V^{*}$ is continuous and dense. We denote by $\|.\|_{H},\|.\|_{V}$, and $\|.\|_{V^{*}}$ the norms in $H, V$, and $V^{*}$, respectively; by $\langle., .\rangle_{V, V^{*}}$ (i.e., $\langle z, v\rangle_{V, V^{*}}=z(v)$ for $\left.z \in V^{*}, v \in V\right)$ the duality product between $V$ and $V^{*}$; and by $\langle., .\rangle_{H}$ the inner product in $H$. The duality product between $V$ and $V^{*}$ has the following property [35]:

$$
\langle\boldsymbol{u}, \boldsymbol{v}\rangle_{V, V^{*}}=\langle\boldsymbol{u}, \boldsymbol{v}\rangle_{H}, \boldsymbol{u} \in H, \boldsymbol{v} \in V .
$$

\subsection{Evolution systems}

Let us consider the nonlinear evolution system on the space $H$ :

$$
\frac{d \boldsymbol{X}(t)}{d t}=\boldsymbol{F}(\boldsymbol{X}(t), t), \boldsymbol{X}\left(t_{0}\right)=\boldsymbol{X}_{0} \in H
$$

where $\boldsymbol{X}$ is assumed to be in $H$ for almost every (a.e.) $t \in\left[t_{0}, \infty\right)$ and $\boldsymbol{F}: H \times\left[t_{0}, \infty\right) \rightarrow V^{*}$ is a family of nonlinear operators defined for a.e. $t \in\left[t_{0}, \infty\right)$. The following definition is a deterministic version of the stochastic one in [36].

Definition 4.1. A H-valued process $\left\{X(t), t \in\left[t_{0}, T\right]\right\}$ is said to be a variational solution of (26) iffor any $\psi \in V$ :

$$
\langle\boldsymbol{X}(t), \boldsymbol{\psi}\rangle_{H}=\left\langle\boldsymbol{X}_{0}, \boldsymbol{\psi}\right\rangle_{H}+\int_{t_{0}}^{t}\langle\boldsymbol{F}(\boldsymbol{X}(s), s), \psi\rangle_{V, V^{*}} d s
$$

for each $t \in\left[t_{0}, T\right]$. If $T$ is replaced by $\infty$, then $X(t), t \geq t_{0}$, is said to be a global variational solution of (26).

The following definition is an extended version of the one in finite dimensional space in [37] to infinite dimensional space.

Definition 4.2. Let $\alpha$ be a class $\mathcal{K}_{\infty}$-function. The variational solution of (26) is said to be

1. globally stable if, for each $\boldsymbol{X}_{0} \in H$, there exists $\delta=\delta\left(\left\|\boldsymbol{X}_{0}\right\|_{H}\right)$ such that $\|\boldsymbol{X}(t)\|_{H} \leq \delta\left(\left\|\boldsymbol{X}_{0}\right\|_{H}\right)$, a.e. $(\boldsymbol{X}, t) \in V \times\left[t_{0}, \infty\right)$

2. globally $\mathcal{K}_{\infty}$-exponentially stable if it is globally stable and $\|\boldsymbol{X}(t)\|_{H} \leq \alpha\left(\left\|\boldsymbol{X}\left(t_{0}\right)\right\|_{H}\right) e^{-c\left(t-t_{0}\right)}$, a. e. $(X, t) \in V \times\left[t_{0}, \infty\right)$, where $c$ is a positive constant depending on the initial condition;

3. globally practically $\mathcal{K}_{\infty}$-exponentially stable if it is globally stable and $\|\boldsymbol{X}(t)\|_{H} \leq \alpha\left(\left\|\boldsymbol{X}\left(t_{0}\right)\right\|_{H}\right) \times$ $e^{-c\left(t-t_{0}\right)}+c_{0}$, a.e. $(X, t) \in V \times\left[t_{0}, \infty\right)$, where $c$ is a positive constant independent of the initial condition, and $c_{0}$ is positive constant.

If $\alpha\left(\left\|\boldsymbol{X}\left(t_{0}\right)\right\|_{H}\right) \equiv\left\|\boldsymbol{X}\left(t_{0}\right)\right\|_{H}$, then " $\mathcal{K}_{\infty}$ " is dropped in the above definition. Moreover, if $c>0$ depends on the initial conditions, then "exponentially" is replaced by asymptotically. 


\subsection{Well-posedness and stability of evolution systems}

We assume that $\boldsymbol{F}: H \times\left[t_{0}, \infty\right) \rightarrow V^{*}$ is measurable and satisfies the following continuity and local monotonicity and growth conditions.

\section{Assumption 4.1.}

1) [Continuity] The mapping $V \ni \boldsymbol{v} \rightarrow \boldsymbol{F}(\boldsymbol{v}, t) \in V^{*}$ is continuous a.e. $t \in\left[t_{0}, \infty\right)$.

2) [Local monotonicity] For any $\boldsymbol{u}, \boldsymbol{v} \in V$ with $\|\boldsymbol{u}\|_{H} \leq \epsilon$ and $\|\boldsymbol{v}\|_{H} \leq \epsilon$, where $\epsilon$ is a positive constant, there exists a constant $c_{\epsilon}$ such that

$$
2\langle\boldsymbol{u}-\boldsymbol{v}, \boldsymbol{F}(\boldsymbol{u}, t)-\boldsymbol{F}(\boldsymbol{v}, t)\rangle_{V, V^{*}} \leq c_{\epsilon}\|\boldsymbol{u}-\boldsymbol{v}\|_{H}^{2},
$$

a.e. $t \in\left[t_{0}, \infty\right)$.

3) [Local growth] There exists a constant $\delta$ such that:

$$
\|\boldsymbol{F}(\boldsymbol{u}, t)\|_{V^{*}} \leq \delta\left(1+\|\boldsymbol{u}\|_{V}^{p-1}\right) \forall \boldsymbol{u} \in V,\|\boldsymbol{u}\|_{V} \leq \epsilon .
$$

Theorem 4.1. Under Assumption 4.1, suppose that there exist a function $U(X, t) \in C^{1}\left(H ;\left[t_{0}, \infty\right)\right)$ referred to as a Lyapunov function, and class $\mathcal{K}_{\infty}$-functions $\alpha_{1}$ and $\alpha_{2}$, and nonnegative constants $\varrho_{1}$ and $\varrho_{2}$ such that

$$
-\varrho_{1}+\alpha_{1}\left(\|\boldsymbol{X}\|_{H}\right) \leq U(\boldsymbol{X}, t) \leq \alpha_{2}\left(\|\boldsymbol{X}\|_{H}\right)+\varrho_{2},
$$

a.e. $(\boldsymbol{X}, t) \in V \times\left[t_{0}, \infty\right)$, and that the generator $\mathcal{L} U:=\frac{d U}{d t}$ given by

$$
\mathcal{L} U(\boldsymbol{X}, t)=U_{t}(\boldsymbol{X}, t)+\left\langle\boldsymbol{F}(\boldsymbol{X}, t), U_{\boldsymbol{X}}(\boldsymbol{X}, t)\right\rangle_{V, V^{*}}
$$

with $U_{t}(\boldsymbol{X}, t)$ and $U_{\boldsymbol{X}}(\boldsymbol{X}, t)$ being the (Fréchet) derivatives of $U(\boldsymbol{X}, t)$ with respect to $t$ and $\boldsymbol{X}$, respectively.

1) [well-posedness] If the generator $\mathcal{L} U(\boldsymbol{X}, t)$ satisfies

$$
\mathcal{L} U(\boldsymbol{X}, t) \leq c(1+U(\boldsymbol{X}, t)), \text { a.e. }(\boldsymbol{X}, t) \in V \times\left[t_{0}, \infty\right),
$$

where $c$ is a nonnegative constant. Then the system (26) is globally well-posed in terms of the variational solution for each $\boldsymbol{X}_{0} \in H$.

2) [stability] If the generator $\mathcal{L} U(X, t)$ satisfies

$$
\mathcal{L} U(\boldsymbol{X}, t) \leq-c_{3}\|\boldsymbol{X}\|_{H}^{2}+c_{0}, \text { a.e. }(\boldsymbol{X}, t) \in V \times\left[t_{0}, \infty\right),
$$

where $c_{3}$ is a positive constant. If $c_{0}=0, \varrho_{1}=0$, and $\varrho_{2}=0$, the equilibrium $\boldsymbol{X} \equiv 0$ is globally $\mathcal{K}_{\infty}$-exponentially stable. If any of $c_{0}, \varrho_{1}$, and $\varrho_{2}$ is a positive constant, the equilibrium $\boldsymbol{X} \equiv 0$ is globally practically $\mathcal{K}_{\infty}$-exponentially stable.

If $\alpha_{1}\left(\|\boldsymbol{X}\|_{H}^{p}\right) \equiv c_{1}\|\boldsymbol{X}\|_{H}^{p}$ and $\alpha_{2}\left(\|\boldsymbol{X}\|_{H}^{p}\right) \equiv c_{2}\|\boldsymbol{X}\|_{H}^{p}$ with $c_{1}$ and $c_{2}$ being positive constants, then " $\mathcal{K}_{\infty}$ " is dropped in the above statement. Moreover, if $c_{3}>0$ depends on the initial conditions, then "exponentially" is replaced by asymptotically.

Proof. See [38]. The above theorem covers most common types of stability of evolution systems. For example, $\frac{d X(t)}{d t}=-X(t)$ is globally exponentially stable while $\frac{d X(t)}{d t}=-X^{3}(t)$ and $\frac{d X(t)}{d t}=-\frac{X(t)}{\sqrt{1+X^{2}(t)}}$ are globally asymptotically stable with a note that $\frac{d X(t)}{d t}=-\frac{X(t)}{\sqrt{1+X^{2}(t)}}$ is also locally exponentially stable. 


\section{Control design}

\subsection{Abstract formulation}

Let $L^{2}(\mathcal{D})$ denote the $L^{2}$-space with the norm $\|.\|_{L^{2}}$ and inner product $\langle., .\rangle_{L^{2}}$ and $W^{m, p}(\mathcal{D})$, with $(m, p)$ being integers, denote the Sobolev space of order $m$ and degree $p$, see [39]. Considering $s \in[0, \Gamma]$ as the parameter defined at every $t \geq t_{0}$, we can regard $\tilde{\boldsymbol{r}}(s, t), \boldsymbol{q}(s, t), \boldsymbol{v}(s, t)$, and $\boldsymbol{\omega}(s, t)$ as $\tilde{\boldsymbol{r}}(t) \in\left(W^{2,2}([0, \Gamma])\right)^{3}, \boldsymbol{q}(t) \in\left(W^{2,2}([0, \Gamma])\right)^{4}, \boldsymbol{v}(t) \in\left(L^{2}([0, \Gamma])\right)^{3}, \boldsymbol{\omega}(t) \in\left(L^{2}([0, \Gamma])\right)^{3}$, respectively. Similarly, $\tilde{\boldsymbol{r}}(0, t), \dot{\tilde{\boldsymbol{r}}}(0, t), \boldsymbol{q}(0, t)$, and $\omega(0, t)$ are regarded as $\tilde{\boldsymbol{r}}_{1}^{B 0}(t) \in \mathbb{R}^{3}, \tilde{\boldsymbol{r}}_{2}^{B 0}(t) \in \mathbb{R}^{3}, \boldsymbol{q}^{B 0}(t) \in \mathbb{R}^{4}$, $\omega^{B 0}(t) \in \mathbb{R}^{3}$, respectively. Moreover, $\tilde{\boldsymbol{r}}(\Gamma, t), \dot{\tilde{\boldsymbol{r}}}(\Gamma, t), \boldsymbol{q}(\Gamma, t)$, and $\boldsymbol{\omega}(\Gamma, t)$ are considered as $\tilde{\boldsymbol{r}}_{1}^{B \Gamma}(t) \in \mathbb{R}^{3}$, $\tilde{\boldsymbol{r}}_{2}^{B \Gamma}(t) \in \mathbb{R}^{3}, \boldsymbol{q}^{B \Gamma}(t) \in \mathbb{R}^{4}, \omega^{B \Gamma}(t) \in \mathbb{R}^{3}$, respectively. Let us also define $\mathbb{D} \phi(s):=\frac{\partial \phi}{\partial s}$. Thus, we can write the beam system (11) in the following evolution system (abstract form):

$$
\begin{aligned}
& \frac{d \tilde{r}_{1}}{d t}=\boldsymbol{R}_{1}(\boldsymbol{q}) \boldsymbol{v}, \\
& \frac{d \boldsymbol{q}}{d t}=\boldsymbol{K}(\boldsymbol{q}) \boldsymbol{\omega}, \\
& m_{0} \frac{d \boldsymbol{v}}{d t}=m_{0}\left(\boldsymbol{R}_{1}^{-1}(\boldsymbol{q})\right)_{t} \boldsymbol{R}_{1}(\boldsymbol{q}) \boldsymbol{v}+\boldsymbol{R}_{1}^{-1}(\boldsymbol{q}) \mathbb{D} \boldsymbol{n}+\boldsymbol{f}_{1}, \\
& \boldsymbol{J}_{0} \frac{d \omega}{d t}=\mathbb{D} \boldsymbol{m}-\boldsymbol{\omega} \times\left(\boldsymbol{J}_{0} \boldsymbol{\omega}\right)+\mathbb{D} \boldsymbol{r} \times \boldsymbol{n}+\boldsymbol{f}_{2} .
\end{aligned}
$$

The initial conditions (14) are written in the abstract form as:

$$
\tilde{\boldsymbol{r}}_{1}\left(t_{0}\right)=\tilde{\boldsymbol{r}}_{10}, \tilde{\boldsymbol{r}}_{2}\left(t_{0}\right)=\tilde{\boldsymbol{r}}_{20}, \boldsymbol{\theta}\left(t_{0}\right)=\boldsymbol{\theta}_{0}, \boldsymbol{\omega}\left(t_{0}\right)=\boldsymbol{\omega}_{0}
$$

The boundary conditions (15) are written as:

$$
\text { At } s=0:\left\{\begin{array}{l}
\frac{d \tilde{\boldsymbol{r}}_{1}^{B 0}}{d t}=\tilde{\boldsymbol{r}}_{2}^{B 0}, \\
\frac{d \boldsymbol{q}^{B 0}}{d t}=\boldsymbol{K}\left(\boldsymbol{q}^{B 0}\right) \boldsymbol{\omega}^{B 0}, \\
m_{P} \frac{d \tilde{\boldsymbol{r}}_{2}^{B 0}}{d t}=\boldsymbol{n}^{B 0}+\boldsymbol{f}_{1}^{B 0}, \\
\boldsymbol{J}_{P} \frac{d \boldsymbol{\omega}^{B 0}}{d t}=\boldsymbol{m}^{B 0}+\boldsymbol{f}_{2}^{B 0},
\end{array} \quad \text { At } s=\Gamma:\left\{\begin{array}{l}
\frac{d \tilde{\boldsymbol{r}}_{1}^{B \Gamma}}{d t}=\tilde{\boldsymbol{r}}_{2}^{B \Gamma}, \\
\frac{d \boldsymbol{q}^{B \Gamma}}{d t}=\boldsymbol{K}\left(\boldsymbol{q}^{B \Gamma}\right) \boldsymbol{\omega}^{B \Gamma}, \\
\boldsymbol{M}_{H} \frac{d \boldsymbol{r}_{2}^{B \Gamma}}{d t}=-\boldsymbol{n}^{B \Gamma}+\boldsymbol{\phi}_{1 B}+\boldsymbol{f}_{1}^{B \Gamma}, \\
\boldsymbol{J}_{H} \frac{d \omega^{B \Gamma}}{d t}=-\boldsymbol{m}^{B \Gamma}+\boldsymbol{\phi}_{2 B}+\boldsymbol{f}_{2}^{B \Gamma},
\end{array}\right.\right.
$$

where $\boldsymbol{m}^{B 0}(t)$ and $\boldsymbol{n}^{B 0}(t)$ are the values of $\boldsymbol{m}(t)$ and $\boldsymbol{n}(t)$ evaluated at $s=0$; and $\boldsymbol{n}^{B \Gamma}(t)$ and $\boldsymbol{m}^{B \Gamma}(t)$ are the values of $\boldsymbol{n}(t)$ and $\boldsymbol{m}(t)$ evaluated at $s=\Gamma$.

\subsection{Control design}

To design the boundary control vectors $\phi_{i B}, i=1,2$, we consider the following Lyapunov functional candidate:

$$
U=U_{0}+U_{1}+U_{B}
$$

where the functionals $U_{0}, U_{1}$, and $U_{2}$ are chosen as follows:

$$
\begin{aligned}
U_{0}= & \frac{m_{0}}{2}\|\boldsymbol{v}\|_{L^{2}}^{2}+\frac{1}{2}\left\langle\boldsymbol{\omega}, \boldsymbol{J}_{0} \boldsymbol{\omega}\right\rangle_{L^{2}}+\sum_{i=1}^{2} G \bar{A}_{i}\left\langle\eta_{i},\left(\frac{1}{2} \eta_{i}-\frac{1}{6} \eta_{i}^{2}+\frac{1}{12} \eta_{i}^{3}\right)\right\rangle_{L^{2}}+E A\left\langle\varepsilon,\left(\frac{1}{2} \varepsilon-\frac{1}{6} \varepsilon^{2}+\frac{1}{12} \varepsilon^{3}\right)\right\rangle_{L^{2}} \\
& +\sum_{i=1}^{2} E I_{i}\left\langle\mu_{i},\left(\frac{1}{2} \mu_{i}-\frac{1}{6} \mu_{i}^{2}+\frac{1}{12} \mu_{i}^{3}\right)\right\rangle_{L^{2}}+G I_{3}\left\langle\mu_{3},\left(\frac{1}{2} \mu_{3}-\frac{1}{6} \mu_{3}^{2}+\frac{1}{12} \mu_{3}^{3}\right)\right\rangle_{L^{2}}, \\
U_{1}= & \gamma m_{0}\left\langle\mathbb{D} \boldsymbol{r}-\varrho \boldsymbol{R}_{1}(\boldsymbol{q}) \mathbb{D} \boldsymbol{r}^{0}, \tilde{\boldsymbol{r}}_{2} s\right\rangle_{L^{2}}+\gamma\left\langle\boldsymbol{\mu}, \boldsymbol{J}_{0} \boldsymbol{\omega} s\right\rangle_{L^{2}}, \\
U_{B}= & \frac{m_{P}}{2}\left\|\tilde{\boldsymbol{r}}_{2}^{B 0}\right\|^{2}+\frac{1}{2}\left(\boldsymbol{\omega}^{B 0}\right)^{T} \boldsymbol{J}_{P} \boldsymbol{\omega}^{B 0}+\frac{1}{2}\left(\gamma_{1} \frac{\tilde{r}_{1}^{B \Gamma}}{\Delta^{B \Gamma}}+\tilde{\boldsymbol{r}}_{2}^{B \Gamma}+\gamma \Gamma \boldsymbol{R}_{1}\left(\boldsymbol{q}^{B \Gamma}\right) \boldsymbol{\vartheta}^{B \Gamma}\right)^{T} \boldsymbol{M}_{H}\left(\gamma_{1} \tilde{r}_{1}^{B \Gamma}+\tilde{\boldsymbol{r}}_{2}^{B \Gamma}+\gamma \Gamma \boldsymbol{R}_{1}\left(\boldsymbol{q}^{B \Gamma}\right) \boldsymbol{\vartheta}^{B \Gamma}\right) \\
& +\frac{1}{2}\left(\gamma_{2} \boldsymbol{K}^{T}\left(\boldsymbol{q}^{B \Gamma}\right) \operatorname{col}\left(q_{1}^{B \Gamma}-1, \overline{\boldsymbol{q}}^{B \Gamma}\right)+\boldsymbol{\omega}^{B \Gamma}+\gamma \Gamma \boldsymbol{\mu}^{B \Gamma}\right)^{T} \boldsymbol{J}_{H}\left(\gamma_{2} \boldsymbol{K}^{T}\left(\boldsymbol{q}^{B \Gamma}\right) \operatorname{col}\left(q_{1}^{B \Gamma}-1, \overline{\boldsymbol{q}}^{B \Gamma}\right)+\boldsymbol{\omega}^{B \Gamma}+\gamma \Gamma \boldsymbol{\mu}^{B \Gamma}\right) \\
& +2 \gamma_{1}\left(\Delta^{B \Gamma}-1\right)+\gamma_{2} k_{2 B}\left\|\operatorname{col}\left(q_{1}^{B \Gamma}-1, \overline{\boldsymbol{q}}^{B \Gamma}\right)\right\|^{2},
\end{aligned}
$$

where $\gamma, \gamma_{1}, \gamma_{2}, k_{2 B}$ are positive constants and $\boldsymbol{K}_{1 B}$ with $\lambda_{m}\left(\boldsymbol{K}_{1 B}\right) \geq 1$ is a positive definite matrix to be chosen later, and $\Delta^{B \Gamma}:=\sqrt{1+\left(\tilde{\boldsymbol{r}}_{1}^{B \Gamma}\right)^{T} \boldsymbol{K}_{1 B} \tilde{\boldsymbol{r}}_{1}^{B \Gamma}}$. The constant $0 \leq \varrho \leq 1$ (to be specified later) is included in $U_{1}$ to handle both large and small bending stiffness relatively to the shear stiffness. 
Remark 5.1. The choice of the Lyapunov function candidate $U$ in (37) with $U_{0}, U_{1}$, and $U_{2}$ being given in (38) is elaborated as follows. The function $U_{0}$ is the sum of kinetic and potential energies of the beam, where the potential energy is referred to as the energy due to elastic deformation. The function $U_{1}$ is motivated by the backstepping method [40], for example with the first equation of (3) and (20) it can be shown that $\frac{d \tilde{r}_{1}}{d t}=\gamma m_{0} \boldsymbol{\vartheta}$ s (with a note that $\mathbb{D} \boldsymbol{r}=\boldsymbol{R}_{1}(\boldsymbol{q}) \boldsymbol{\vartheta}+\boldsymbol{R}_{1}(\boldsymbol{q}) \mathbb{D} \boldsymbol{r}^{0}$, see (3)) is globally exponentially stable at the origin by an appropriate boundary control. The function $U_{B}$ puts appropriate weights on translational displacements and velocities of the beam at the boundaries. The translational and rotational displacements at the left-end are not included in $U_{B}$ while fairly complicated motions at the right-end are included in $U_{B}$ to avoid difficulties in the control design and stability analysis, see also Remark 3.1. Finally, the term $\gamma_{1} \frac{\tilde{\boldsymbol{r}}_{1}^{B \Gamma}}{\Delta^{B \Gamma}}$ is used in $U_{B}$ instead of $\gamma_{1} \tilde{\boldsymbol{r}}_{1}^{B \Gamma}$ to overcome impossibility in calculating the upper-bound of the generator $\mathcal{L} U_{B}$.

We now find the bounds of $U$. Using $\frac{1}{3} x^{2}+\frac{1}{12} x^{4} \leq \frac{1}{2}\left(x^{2}-\frac{1}{3} x^{3}+\frac{1}{4} x^{4}\right) \leq \frac{2}{3} x^{2}+\frac{1}{6} x^{4}$ for all $x \in \mathbb{R}$, we can bound $U_{0}$ as:

$$
\begin{aligned}
U_{0} \geq & \frac{m_{0}}{2}\|\boldsymbol{v}\|_{L^{2}}^{2}+\frac{\lambda_{m}\left(\boldsymbol{J}_{0}\right)}{2}\|\omega\|_{L^{2}}^{2}+\frac{1}{3}\left(G \bar{A}_{1} \wedge G \bar{A}_{2} \wedge E A\right)\|\boldsymbol{\vartheta}\|_{L^{2}}^{2}+\frac{1}{12}\left(G \bar{A}_{1} \wedge G \bar{A}_{2} \wedge E A\right)\left\langle 1, \eta_{1}^{4}+\eta_{2}^{4}+\varepsilon^{4}\right\rangle_{L^{2}} \\
& +\frac{1}{3}\left(E I_{1} \wedge E I_{2} \wedge G I_{3}\right)\|\boldsymbol{\mu}\|_{L^{2}}^{2}+\frac{1}{12}\left(E I_{1} \wedge E I_{2} \wedge G I_{3}\right)\left\langle 1, \mu_{1}^{4}+\mu_{2}^{4}+\mu_{3}^{4}\right\rangle_{L^{2}}, \\
U_{0} \leq & \frac{m_{0}}{2}\|\boldsymbol{v}\|_{L^{2}}^{2}+\frac{\lambda_{M}\left(\boldsymbol{J}_{0}\right)}{2}\|\omega\|_{L^{2}}^{2}+\frac{2}{3}\left(G \bar{A}_{1} \vee G \bar{A}_{2} \vee E A\right)\|\boldsymbol{\vartheta}\|_{L^{2}}^{2}+\frac{1}{6}\left(G \bar{A}_{1} \vee G \bar{A}_{2} \vee E A\right)\left\langle 1, \eta_{1}^{4}+\eta_{2}^{4}+\varepsilon^{4}\right\rangle_{L^{2}} \\
& +\frac{2}{3}\left(E I_{1} \vee E I_{2} \vee G I_{3}\right)\|\boldsymbol{\mu}\|_{L^{2}}^{2}+\frac{1}{6}\left(E I_{1} \vee E I_{2} \vee G I_{3}\right)\left\langle 1, \mu_{1}^{4}+\mu_{2}^{4}+\mu_{3}^{4}\right\rangle_{L^{2}},
\end{aligned}
$$

where $\lambda_{m}(\bullet)$ and $\lambda_{M}(\bullet)$ denote the minimum and maximum eigenvalues of $\bullet$, respectively. Using $\mathbb{D} \boldsymbol{r}=\boldsymbol{R}_{1}(\boldsymbol{q}) \boldsymbol{\vartheta}+\boldsymbol{R}_{1}(\boldsymbol{q}) \mathbb{D} \boldsymbol{r}^{0},(7)$, and Young's inequality, we can find the bound of $\left|U_{1}\right|$ as follows

$$
\left|U_{1}\right| \leq \gamma \Gamma m_{0}\left(\varrho_{01}+(1-\varrho) \varrho_{03}\right)\|\boldsymbol{v}\|_{L^{2}}^{2}+\frac{\gamma \Gamma m_{0}}{4 \varrho_{01}}\|\boldsymbol{\vartheta}\|_{L^{2}}^{2}+\gamma \Gamma \lambda_{M}\left(\boldsymbol{J}_{0}\right) \varrho_{02}\|\boldsymbol{\omega}\|_{L^{2}}^{2}+\frac{\gamma \Gamma \lambda_{M}\left(\boldsymbol{J}_{0}\right)}{4 \varrho_{02}}\|\boldsymbol{\mu}\|_{L^{2}}^{2}+\frac{\gamma \Gamma m_{0}(1-\varrho)}{4 \varrho_{03}},
$$

where $\varrho_{0 i}, i=1,2,3$ are positive constants to be determined. The bounds of $U_{2}$ can be calculated as

$$
\begin{aligned}
U_{B} & \geq \frac{m_{P}}{2}\left\|\tilde{\boldsymbol{r}}_{2}^{B 0}\right\|^{2}+\frac{\lambda_{m}\left(\boldsymbol{J}_{P}\right)}{2}\left\|\boldsymbol{\omega}^{B 0}\right\|^{2}+\frac{\lambda_{m}\left(\boldsymbol{M}_{H}\right)}{2}\left\|\gamma_{1} \frac{\tilde{\boldsymbol{r}}_{1}^{B \Gamma}}{\Delta^{B \Gamma}}+\tilde{\boldsymbol{r}}_{2}^{B \Gamma}+\gamma \Gamma \boldsymbol{R}_{1}\left(\boldsymbol{q}^{B \Gamma}\right) \boldsymbol{\boldsymbol { \vartheta } ^ { B \Gamma }}\right\|^{2}+2 \gamma_{1}\left(\Delta^{B \Gamma}-1\right) \\
& +\frac{\lambda_{m}\left(\boldsymbol{J}_{H}\right)}{2}\left\|\gamma_{2} \boldsymbol{K}^{T}\left(\boldsymbol{q}^{B \Gamma}\right) \operatorname{col}\left(q_{1}^{B \Gamma}-1, \overline{\boldsymbol{q}}^{B \Gamma}\right)+\boldsymbol{\omega}^{B \Gamma}+\gamma \Gamma \boldsymbol{\mu}^{B \Gamma}\right\|^{2}+\gamma_{2} k_{2 B}\left\|\operatorname{col}\left(q_{1}^{B \Gamma}-1, \overline{\boldsymbol{q}}^{B \Gamma}\right)\right\|^{2}, \\
U_{B} \leq & \frac{m_{P}}{2}\left\|\tilde{\boldsymbol{r}}_{2}^{B 0}\right\|^{2}+\frac{\lambda_{M}\left(\boldsymbol{J}_{P}\right)}{2}\left\|\boldsymbol{\omega}^{B 0}\right\|^{2}+\frac{\lambda_{M}\left(\boldsymbol{M}_{H}\right)}{2}\left\|\gamma_{1} \frac{\tilde{r}_{1}^{B \Gamma}}{\Delta^{B \Gamma}}+\tilde{\boldsymbol{r}}_{2}^{B \Gamma}+\gamma \Gamma \boldsymbol{R}_{1}\left(\boldsymbol{q}^{B \Gamma}\right) \boldsymbol{\vartheta}^{B \Gamma}\right\|^{2}+2 \gamma_{1}\left(\Delta^{B \Gamma}-1\right) \\
& +\frac{\lambda_{M}\left(\boldsymbol{J}_{H}\right)}{2}\left\|\gamma_{2} \boldsymbol{K}^{T}\left(\boldsymbol{q}^{B \Gamma}\right) \operatorname{col}\left(q_{1}^{B \Gamma}-1, \overline{\boldsymbol{q}}^{B \Gamma}\right)+\boldsymbol{\omega}^{B \Gamma}+\gamma \Gamma \boldsymbol{\mu}^{B \Gamma}\right\|^{2}+\gamma_{2} k_{2 B}\left\|\operatorname{col}\left(q_{1}^{B \Gamma}-1, \overline{\boldsymbol{q}}^{B \Gamma}\right)\right\|^{2},
\end{aligned}
$$

Using (39), (40), (41), and $U_{0}-\left|U_{1}\right|+U_{B} \leq U \leq U_{0}+\left|U_{1}\right|+U_{B}$, we can bound $U$ as follows:

$$
-\varrho_{0}+c_{1} \mathcal{E} \leq U \leq c_{2} \mathcal{E}+\varrho_{0}
$$

where $\mathcal{E}$ is defined in (19), and the constants $c_{1}, c_{2}$, and $\varrho_{0}$ are given by

$$
\begin{aligned}
c_{1}= & {\left[\frac{m_{0}}{2}-\gamma \Gamma m_{0}\left(\varrho_{01}+(1-\varrho) \varrho_{03}\right)\right] \wedge\left[\frac{\lambda_{m}\left(\boldsymbol{J}_{0}\right)}{2}-\gamma \Gamma \varrho_{02} \lambda_{M}\left(\boldsymbol{J}_{0}\right)\right] \wedge\left[\frac{1}{3}\left(E A \wedge G \bar{A}_{1} \wedge G \bar{A}_{2}\right)-\frac{\gamma \Gamma m_{0}}{2 \varrho_{01}}\right] } \\
& \wedge \frac{1}{12}\left(G \bar{A}_{1} \wedge G \bar{A}_{2} \wedge E A\right) \wedge\left[\frac{1}{3}\left(E I_{1} \wedge E I_{2} \wedge G I_{3}\right)-\frac{\gamma \Gamma \lambda_{M}\left(\boldsymbol{J}_{0}\right)}{4 \varrho_{02}}\right] \wedge \frac{1}{12}\left(E I_{1} \wedge E I_{2} \wedge G I_{3}\right) \wedge\left[\frac{\lambda_{m}\left(\boldsymbol{M}_{2 H}\right)}{2}\right] \\
& \wedge \frac{m_{P}}{2} \wedge \frac{\lambda_{m}\left(\boldsymbol{J}_{P}\right)}{2} \wedge \frac{\lambda_{m}\left(\boldsymbol{M}_{H}\right)}{2} \wedge \frac{\lambda_{m}\left(\boldsymbol{J}_{H}\right)}{2} \wedge 2 \gamma_{1} \wedge \gamma_{2} k_{2 B}, \\
c_{2}= & {\left[\frac{m_{0}}{2}+\gamma \Gamma m_{0}\left(\varrho_{01}+(1-\varrho) \varrho_{03}\right)\right] \vee\left[\frac{\lambda_{M}\left(\boldsymbol{J}_{0}\right)}{2}+\gamma \Gamma \varrho_{02} \lambda_{M}\left(\boldsymbol{J}_{0}\right)\right] \vee\left[\frac{2}{3}\left(E A \vee G \bar{A}_{1} \vee G \bar{A}_{2}\right)+\frac{\gamma \Gamma m_{0}}{2 \varrho_{01}}\right] } \\
& \vee \frac{1}{6}\left(G \bar{A}_{1} \vee G \bar{A}_{2} \vee E A\right) \vee\left[\frac{2}{3}\left(E I_{1} \vee E I_{2} \vee G I_{3}\right)+\frac{\gamma \Gamma \lambda_{M}\left(\boldsymbol{J}_{0}\right)}{4 \varrho_{02}}\right] \vee \frac{1}{6}\left(E I_{1} \vee E I_{2} \vee G I_{3}\right) \vee\left[\frac{\lambda_{M}\left(\boldsymbol{M}_{2 H}\right)}{2}\right] \\
& \vee \frac{m_{P}}{2} \vee \frac{\lambda_{M}\left(\boldsymbol{J}_{P}\right)}{2} \vee \frac{\lambda_{M}\left(\boldsymbol{M}_{H}\right)}{2} \vee \frac{\lambda_{M}\left(\boldsymbol{J}_{H}\right)}{2} \vee 2 \gamma_{1} \vee \gamma_{2} k_{2 B}, \\
\varrho_{0} & =\frac{\gamma \Gamma m_{0}(1-\varrho)}{4 \varrho_{03}} .
\end{aligned}
$$

The constants $\gamma, \varrho_{0 i}, i=1,2,3$, and $\varrho$ are chosen such that

$$
c_{1} \geq c_{1}^{\diamond},
$$


where $c_{1}^{\diamond}$ is a strictly positive constant. This is always possible by choosing a small $\gamma$ for given $\varrho_{0 i}, i=1,2,3$, and $\varrho$. Thus, $U$ is a proper functional of $\mathcal{E}$.

We now calculate the infinite generator $\mathcal{L} U$. It is obvious from (37) that

$$
\mathcal{L} U=\mathcal{L} U_{0}+\mathcal{L} U_{1}+\mathcal{L} U_{B},
$$

where $\mathcal{L} U_{0}, \mathcal{L} U_{1}$, and $\mathcal{L} U_{B}$ are detailed in what follows.

Calculation of $\mathcal{L} U_{0}$ : Differentiating $U_{0}$ given in (38) along the solutions of (34), and using integration by parts results in:

$$
\begin{aligned}
\mathcal{L} U_{0} & =m_{0}\left\langle\boldsymbol{v},\left(\boldsymbol{R}_{1}^{-1}(\boldsymbol{q})\right)_{t} \boldsymbol{R}_{1}(\boldsymbol{q}) \boldsymbol{v}\right\rangle_{L^{2}}+\left\langle\boldsymbol{v}, \boldsymbol{R}_{1}^{-1}(\boldsymbol{q}) \mathbb{D} \boldsymbol{n}\right\rangle_{L^{2}}+\left\langle\boldsymbol{v}, \boldsymbol{f}_{1}\right\rangle_{L^{2}}+\langle\boldsymbol{\omega}, \mathbb{D} \boldsymbol{m}\rangle_{L^{2}}-\left\langle\boldsymbol{\omega}, \boldsymbol{\omega} \times\left(\boldsymbol{J}_{0} \boldsymbol{\omega}\right)\right\rangle_{L^{2}} \\
& +\langle\boldsymbol{\omega}, \mathbb{D} \boldsymbol{r} \times \boldsymbol{n}\rangle_{L^{2}}+\left\langle\boldsymbol{\omega}, \boldsymbol{f}_{2}\right\rangle_{L^{2}}+\sum_{i=1}^{2} G \bar{A}_{i}\left\langle\eta_{i t},\left(\eta_{i}-\frac{1}{2} \eta_{i}^{2}+\frac{1}{3} \eta_{i}^{3}\right)\right\rangle_{L^{2}}+E A\left\langle\varepsilon_{t},\left(\varepsilon-\frac{1}{2} \varepsilon^{2}+\frac{1}{3} \varepsilon^{3}\right)\right\rangle_{L^{2}} \\
& +\sum_{i=1}^{2} E I_{i}\left\langle\mu_{i t},\left(\mu_{i}-\frac{1}{2} \mu_{i}^{2}+\frac{1}{3} \mu_{i}^{3}\right)\right\rangle_{L^{2}}+G I_{3}\left\langle\mu_{3 t},\left(\mu_{3}-\frac{1}{2} \mu_{3}^{2}+\frac{1}{3} \mu_{3}^{3}\right)\right\rangle_{L^{2}} .
\end{aligned}
$$

To further calculate $\mathcal{L} U_{0}$, we note the following facts:

$$
\begin{aligned}
& \left\langle\boldsymbol{v},\left(\boldsymbol{R}_{1}^{-1}(\boldsymbol{q})\right)_{t} \boldsymbol{R}_{1}(\boldsymbol{q}) \boldsymbol{v}\right\rangle_{L^{2}}=0, \\
& \left\langle\omega, \omega \times\left(J_{0} \omega\right)\right\rangle_{L^{2}}=0, \\
& \left\langle\boldsymbol{v}, \boldsymbol{R}_{1}^{-1}(\boldsymbol{q}) \mathbb{D} \boldsymbol{n}\right\rangle_{L^{2}}=\left\langle\tilde{\boldsymbol{r}}_{t}, \mathbb{D} \boldsymbol{n}\right\rangle_{L^{2}} \\
& =\left(\boldsymbol{n}^{B \Gamma}\right)^{T} \tilde{\boldsymbol{r}}^{B \Gamma}-\left(\boldsymbol{n}^{B 0}\right)^{T} \tilde{\boldsymbol{r}}^{B 0}-\left\langle\boldsymbol{n}, \mathbb{D} \tilde{\boldsymbol{r}}_{t}\right\rangle_{L^{2}} \\
& =\left(\boldsymbol{n}^{B \Gamma}\right)^{T} \tilde{\boldsymbol{r}}^{B \Gamma}-\left(\boldsymbol{n}^{B 0}\right)^{T} \tilde{\boldsymbol{r}}^{B 0}-\langle\boldsymbol{\omega}, \mathbb{D} \boldsymbol{r} \times \boldsymbol{n}\rangle_{L^{2}}-\sum_{i=1}^{2} G \bar{A}_{i}\left\langle\eta_{i t},\left(\eta_{i}-\frac{1}{2} \eta_{i}^{2}+\frac{1}{3} \eta_{i}^{3}\right)\right\rangle_{L^{2}} \\
& -E A\left\langle\varepsilon_{t},\left(\varepsilon-\frac{1}{2} \varepsilon^{2}+\frac{1}{3} \varepsilon^{3}\right)\right\rangle_{L^{2}}, \\
& \langle\boldsymbol{\omega}, \mathbb{D} \boldsymbol{m}\rangle_{L^{2}}=\left(\boldsymbol{m}^{B \Gamma}\right)^{T} \boldsymbol{\omega}^{B \Gamma}-\left(\boldsymbol{m}^{B 0}\right)^{T} \boldsymbol{\omega}^{B 0}-\langle\boldsymbol{m}, \mathbb{D} \boldsymbol{\omega}\rangle_{L^{2}} \\
& =\left(\boldsymbol{m}^{B \Gamma}\right)^{T} \boldsymbol{\omega}^{B \Gamma}-\left(\boldsymbol{m}^{B 0}\right)^{T} \boldsymbol{\omega}^{B 0}-\sum_{i=1}^{2} E I_{i}\left\langle\mu_{i t},\left(\mu_{i}-\frac{1}{2} \mu_{i}^{2}+\frac{1}{3} \mu_{i}^{3}\right)\right\rangle_{L^{2}}+G I_{3}\left\langle\mu_{3 t},\left(\mu_{3}-\frac{1}{2} \mu_{3}^{2}+\frac{1}{3} \mu_{3}^{3}\right)\right\rangle_{L^{2}},
\end{aligned}
$$

where we have used equalities 1) and 4) in Lemma 3.1, see (21), and integration by parts to obtain the last two equations. Substituting (47) into (46) results in

$$
\mathcal{L} U_{0}=\left[\left(\boldsymbol{n}^{B \Gamma}\right)^{T} \tilde{\boldsymbol{r}}_{2}^{B \Gamma}-\left(\boldsymbol{n}^{B 0}\right)^{T} \tilde{\boldsymbol{r}}_{2}^{B 0}\right]+\left[\left(\boldsymbol{m}^{B \Gamma}\right)^{T} \boldsymbol{\omega}^{B \Gamma}-\left(\boldsymbol{m}^{B 0}\right)^{T} \boldsymbol{\omega}^{B 0}\right]+\left\langle\boldsymbol{v}, \boldsymbol{f}_{1}\right\rangle_{L^{2}}+\left\langle\boldsymbol{\omega}, \boldsymbol{f}_{2}\right\rangle_{L^{2}} .
$$

Calculation of $\mathcal{L} U_{1}$ : This is the most complicated and difficult task in the control design. Thus, detailed calculation of $\mathcal{L} U_{1}$ is given. We use the fact that $\langle\boldsymbol{\vartheta}, \boldsymbol{v} s\rangle_{L^{2}}=\left\langle\boldsymbol{R}_{1}(\boldsymbol{q}) \boldsymbol{\vartheta}, \tilde{\boldsymbol{r}}_{t} s\right\rangle_{L^{2}}$ because of (7) and then differentiate $U_{1}$ given in (38) along the solutions of (34) to obtain:

$$
\mathcal{L} U_{1}=\mathcal{L} U_{11}+\mathcal{L} U_{12}+\mathcal{L} U_{21}+\mathcal{L} U_{22}
$$

where

$$
\begin{aligned}
& \mathcal{L} U_{11}=\gamma m_{0}\left\langle\left(\mathbb{D} \boldsymbol{r}-\varrho \boldsymbol{R}_{1}(\boldsymbol{q}) \mathbb{D} \boldsymbol{r}^{0}\right)_{t}, \tilde{\boldsymbol{r}}_{t} s\right\rangle_{L^{2}}, \quad \mathcal{L} U_{12}=\gamma m_{0}\left\langle\left(\mathbb{D} \boldsymbol{r}-\varrho \boldsymbol{R}_{1}(\boldsymbol{q}) \mathbb{D} \boldsymbol{r}^{0}\right), \tilde{\boldsymbol{r}}_{t t} s\right\rangle_{L^{2}}, \\
& \mathcal{L} U_{21}=\gamma\left\langle\boldsymbol{\mu}_{t}, \boldsymbol{J}_{0} \omega s\right\rangle_{L^{2}}, \quad \mathcal{L} U_{22}=\gamma\left\langle\boldsymbol{\mu}, \boldsymbol{J}_{0} \boldsymbol{\omega}_{t} s\right\rangle_{L^{2}} .
\end{aligned}
$$

We now calculate $\mathcal{L} U_{i j},(i, j)=1,2$. We can calculate $\mathcal{L} U_{11}$ as follows:

$$
\begin{aligned}
\mathcal{L} U_{11} & =\left\langle\left(\mathbb{D} \boldsymbol{r}-\varrho \boldsymbol{R}_{1}(\boldsymbol{q}) \mathbb{D} \boldsymbol{r}^{0}\right)_{t}, \tilde{\boldsymbol{r}}_{t} s\right\rangle_{L^{2}} \\
& =\frac{\gamma \Gamma m_{0}}{2}\left\|\tilde{\boldsymbol{r}}_{2}^{B \Gamma}\right\|^{2}-\frac{\gamma m_{0}}{2}\left\|\tilde{\boldsymbol{r}}_{2}\right\|_{L^{2}}^{2}-\gamma m_{0}\left\langle\boldsymbol{R}_{1 t}(\boldsymbol{q}) \mathbb{D} \boldsymbol{r}^{0}, \tilde{\boldsymbol{r}}_{2}\right\rangle_{L^{2}} \\
& \leq \frac{\gamma \Gamma m_{0}}{2}\left\|\tilde{\boldsymbol{r}}_{2}^{B \Gamma}\right\|^{2}-\frac{\gamma m_{0}}{2}\left\|\tilde{\boldsymbol{r}}_{2}\right\|_{L^{2}}^{2}+\gamma \Gamma m_{0} \varrho \epsilon_{11}\|\boldsymbol{\omega}\|_{L^{2}}^{2}+\frac{\gamma \Gamma m_{0} \varrho}{4 \epsilon_{11}}\left\|\tilde{\boldsymbol{r}}_{2}\right\|_{L^{2}}^{2}
\end{aligned}
$$

where $\epsilon_{11}$ is a positive constant to be chosen, and we have used $\mathbb{D} \boldsymbol{r}^{0}=\operatorname{col}(0,0,1)$ and the second equation of (34). Using the third equation of (34) and integration by parts, we can calculate $\mathcal{L} U_{12}$ as 
follows:

$$
\begin{aligned}
\mathcal{L} U_{12}= & \gamma\left\langle\mathbb{D} \boldsymbol{r}-\varrho \boldsymbol{R}_{1}(\boldsymbol{q}) \mathbb{D} \boldsymbol{r}^{0},\left(\mathbb{D} \boldsymbol{n}+\boldsymbol{R}_{1}(\boldsymbol{q}) \boldsymbol{f}_{1}\right) s\right\rangle_{L^{2}} \\
= & \gamma\langle\mathbb{D} \boldsymbol{r}, \mathbb{D} \boldsymbol{n} s\rangle_{L^{2}}-\gamma \varrho\left\langle\boldsymbol{R}_{1}(\boldsymbol{q}) \mathbb{D} \boldsymbol{r}^{0}, \mathbb{D} \boldsymbol{n} s\right\rangle_{L^{2}}+\gamma\left\langle\boldsymbol{\vartheta}+(1-\varrho) \mathbb{D} \boldsymbol{r}^{0}, \boldsymbol{f}_{1} s\right\rangle_{L^{2}} \\
= & \gamma \Gamma\left(\boldsymbol{n}^{B \Gamma}\right)^{T} \mathbb{D} \boldsymbol{r}^{B \Gamma}-\gamma \Gamma G \sum_{i=1}^{2} \bar{A}_{i}\left[\frac{1}{2}\left(\eta_{i}^{B \Gamma}\right)^{2}-\frac{1}{6}\left(\eta_{i}^{B \Gamma}\right)^{3}+\frac{1}{12}\left(\eta_{i}^{B \Gamma}\right)^{4}\right]-\gamma \Gamma E A\left[\frac{1}{2}\left(\varepsilon^{B \Gamma}\right)^{2}-\frac{1}{6}\left(\varepsilon^{B \Gamma}\right)^{3}+\frac{1}{12}\left(\varepsilon^{B \Gamma}\right)^{4}\right] \\
& -\gamma G \sum_{i=1}^{2} \bar{A}_{i}\left\langle 1, \frac{1}{2} \eta_{i}^{2}-\frac{1}{3} \eta_{i}^{3}+\frac{1}{4} \eta_{i}^{4}\right\rangle_{L^{2}}-\gamma E A\left\langle 1, \frac{1}{2} \varepsilon^{2}-\frac{1}{3} \varepsilon^{3}+\frac{1}{4} \varepsilon^{4}\right\rangle_{L^{2}}-\gamma\left\langle\boldsymbol{n}, \boldsymbol{b}_{3}\right\rangle_{L^{2}}-\gamma\langle\boldsymbol{n},(\boldsymbol{\mu} \times \mathbb{D} \boldsymbol{r}) s\rangle_{L^{2}} \\
& -\gamma \Gamma \varrho\left(\boldsymbol{n}^{B \Gamma}\right)^{T} \boldsymbol{R}_{1}\left(\boldsymbol{q}^{B \Gamma}\right) \mathbb{D} \boldsymbol{r}^{0}+\gamma \varrho\left\langle\boldsymbol{n}, \boldsymbol{R}_{1}(\boldsymbol{q}) \mathbb{D} \boldsymbol{r}^{0}\right\rangle_{L^{2}}+\gamma \varrho\left\langle\boldsymbol{n}, \mathbb{D}\left(\boldsymbol{R}_{1}(\boldsymbol{q}) \mathbb{D} \boldsymbol{r}^{0}\right) s\right\rangle_{L^{2}}+\gamma\left\langle\boldsymbol{\vartheta}+(1-\varrho) \mathbb{D} \boldsymbol{r}^{0}, \boldsymbol{f}_{1} s\right\rangle_{L^{2}} .
\end{aligned}
$$

To further calculate $\mathcal{L} U_{12}$, we observe the following facts by using (12) and (13), and Young's inequality:

$$
\begin{aligned}
& \boldsymbol{R}_{1}(\boldsymbol{q}) \mathbb{D} \boldsymbol{r}^{0}=\boldsymbol{b}_{3}, \\
& \left\langle\boldsymbol{n}, \boldsymbol{b}_{3}\right\rangle_{L^{2}} \leq E A \bar{\epsilon}_{12}\left\langle 1, \varepsilon^{2}\right\rangle_{L^{2}}+E A\left(\bar{\epsilon}_{13}+\frac{\bar{\epsilon}_{13}^{4 / 3}}{4}\right)\left\langle 1, \varepsilon^{4}\right\rangle_{L^{2}}+E A \Gamma\left(\frac{1}{4 \bar{\epsilon}_{12}}+\frac{1}{16 \bar{\epsilon}_{13}}+\frac{1}{12 \bar{\epsilon}_{13}^{4}}\right), \\
& \left\langle\boldsymbol{n}, \mathbb{D}\left(\boldsymbol{R}_{1}(\boldsymbol{q}) \mathbb{D} \boldsymbol{r}^{0}\right) s\right\rangle_{L^{2}}=\left\langle\boldsymbol{n}, \mathbb{D} \boldsymbol{b}_{3} s\right\rangle_{L^{2}}=\left\langle\boldsymbol{n},\left(\boldsymbol{\mu} \times \boldsymbol{b}_{3}\right) s\right\rangle_{L^{2}} \\
& \leq \Gamma\left\langle 1,\left|Q_{1} \mu_{1}\right|+\left|Q_{2} \mu_{2}\right|\right\rangle_{L^{2}} \\
& \leq \Gamma G \sum_{i=1}^{2} \bar{A}_{i}\left\langle 1, \epsilon_{12} \eta_{i}^{2}+\left(\epsilon_{13}+\frac{1}{4} \epsilon_{13}^{\frac{4}{3}}\right) \eta_{i}^{4}\right\rangle_{L^{2}}+\Gamma\left(G \bar{A}_{1} \vee G \bar{A}_{2}\right)\left(\frac{1}{4 \epsilon_{12}}+\frac{1}{16 \epsilon_{13}}\right)\left\langle 1, \mu_{1}^{2}+\mu_{2}^{2}\right\rangle_{L^{2}} \\
& +\Gamma\left(G \bar{A}_{1} \vee G \bar{A}_{2}\right) \frac{1}{12 \epsilon_{13}^{4}}\left\langle 1, \mu_{1}^{4}+\mu_{2}^{4}\right\rangle_{L^{2}} \text {, }
\end{aligned}
$$

where $\epsilon_{12}, \epsilon_{13}, \bar{\epsilon}_{12}$, and $\bar{\epsilon}_{13}$ are positive constants to be chosen. Substituting (53) into (52) gives

$$
\begin{aligned}
\mathcal{L} U_{12} & \leq-\gamma \Gamma G \sum_{i=1}^{2} \bar{A}_{i}\left[\frac{1}{2}\left(\eta_{i}^{B \Gamma}\right)^{2}-\frac{1}{6}\left(\eta_{i}^{B \Gamma}\right)^{3}+\frac{1}{12}\left(\eta_{i}^{B \Gamma}\right)^{4}\right]-\gamma \Gamma E A\left[\frac{1}{2}\left(\varepsilon^{B \Gamma}\right)^{2}-\frac{1}{6}\left(\varepsilon^{B \Gamma}\right)^{3}+\frac{1}{12}\left(\varepsilon^{B \Gamma}\right)^{4}\right] \\
& +\gamma \Gamma\left(\boldsymbol{n}^{B \Gamma}\right)^{T} \boldsymbol{R}_{1}\left(\boldsymbol{q}^{B \Gamma}\right) \boldsymbol{\vartheta}^{B \Gamma}+(1-\varrho) \gamma \Gamma\left(\boldsymbol{n}^{B \Gamma}\right)^{T} \boldsymbol{R}_{1}\left(\boldsymbol{q}^{B \Gamma}\right) \boldsymbol{r}^{0}-\gamma G \sum_{i=1}^{2} \bar{A}_{i}\left\langle 1, \frac{1}{2} \eta_{i}^{2}-\frac{1}{3} \eta_{i}^{3}+\frac{1}{4} \eta_{i}^{4}\right\rangle_{L^{2}} \\
& -\gamma E A\left\langle 1, \frac{1}{2} \varepsilon^{2}-\frac{1}{3} \varepsilon^{3}+\frac{1}{4} \varepsilon^{4}\right\rangle_{L^{2}}+(1-\varrho) E A\left(\bar{\epsilon}_{12} \varepsilon^{2}+\left(\bar{\epsilon}_{13}+\frac{\bar{\epsilon}_{13}^{4 / 3}}{4}\right) \varepsilon^{4}\right) \\
& +\gamma \Gamma \varrho G \sum_{i=1}^{2} \bar{A}_{i}\left\langle 1, \epsilon_{12} \eta_{i}^{2}+\left(\epsilon_{13}+\frac{1}{4} \epsilon_{13}^{\frac{4}{3}}\right) \eta_{i}^{4}\right\rangle_{L^{2}}+\gamma \Gamma \varrho\left(G \bar{A}_{1} \vee G \bar{A}_{2}\right)\left(\frac{1}{4 \epsilon_{12}}+\frac{1}{16 \epsilon_{13}}\right)\left\langle 1, \mu_{1}^{2}+\mu_{2}^{2}\right\rangle_{L^{2}} \\
& +\gamma \Gamma \varrho\left(G \bar{A}_{1} \vee G \bar{A}_{2}\right) \frac{1}{12 \epsilon_{13}^{4}}\left\langle 1, \mu_{1}^{4}+\mu_{2}^{4}\right\rangle_{L^{2}}-\gamma\langle\boldsymbol{n},(\boldsymbol{\mu} \times \mathbb{D} \boldsymbol{r}) s\rangle_{L^{2}}+\gamma\left\langle\boldsymbol{\vartheta}+(1-\varrho) \mathbb{D} \boldsymbol{r}^{0}, \boldsymbol{f}_{1} s\right\rangle_{L^{2}} \\
& +(1-\varrho) E A \Gamma\left(\frac{1}{4 \bar{\epsilon}_{12}}+\frac{1}{16 \bar{\epsilon}_{13}}+\frac{1}{12 \bar{\epsilon}_{13}^{4}}\right) .
\end{aligned}
$$

It is noted that $\boldsymbol{\mu}_{t}=\mu_{1 t} \boldsymbol{b}_{1}+\mu_{2 t} \boldsymbol{b}_{2}+\mu_{3 t} \boldsymbol{b}_{3}+\boldsymbol{\omega} \times \boldsymbol{\mu}$. Using this equation and the first equation of (21), we can calculate $\mathcal{L} U_{21}$ as follows

$$
\begin{aligned}
\mathcal{L} U_{21} & =\gamma\left\langle\mathbb{D} \boldsymbol{\omega}, \boldsymbol{J}_{0} \boldsymbol{\omega}\right\rangle_{L^{2}}+\gamma\left\langle\boldsymbol{\omega} \times \boldsymbol{\mu}, \boldsymbol{J}_{0} \boldsymbol{\omega} s\right\rangle_{L^{2}} \\
& \leq \gamma \Gamma \lambda_{M}\left(\boldsymbol{J}_{0}\right)\left\|\boldsymbol{\omega}^{B \Gamma}\right\|^{2}-\frac{\gamma \lambda_{m}\left(\boldsymbol{J}_{0}\right)}{2}\|\boldsymbol{\omega}\|_{L^{2}}^{2}+\gamma \Gamma \boldsymbol{J}_{0 M} \epsilon_{14}\|\boldsymbol{\mu}\|_{L^{2}}^{2}+\gamma \Gamma J_{0 M} \frac{1}{4 \epsilon_{14}}\left\langle\omega_{1}^{4}+\omega_{2}^{4}+\omega_{3}^{4}\right\rangle_{L^{2}},
\end{aligned}
$$

where

$$
J_{0 M}:=\left|J_{01}-J_{02}\right| \vee\left|J_{01}-J_{03}\right| \vee\left|J_{02}-J_{03}\right|
$$

and $\epsilon_{14}$ is a positive constant to be determined. Using the last equation of (34) and the fact that $\mathbb{D} \boldsymbol{\mu}=\mathbb{D} \mu_{1} \boldsymbol{b}_{1}+\mathbb{D} \mu_{2} \boldsymbol{b}_{2}+\mathbb{D} \mu_{3} \boldsymbol{b}_{3}$, we can calculate $\mathcal{L} U_{22}$ as follows:

$$
\begin{aligned}
\mathcal{L} U_{22}= & \gamma\langle\boldsymbol{\mu}, \mathbb{D} \boldsymbol{m} s\rangle_{L^{2}}-\gamma\left\langle\boldsymbol{\mu}, \boldsymbol{\omega} \times\left(\boldsymbol{J}_{0} \boldsymbol{\omega}\right) s\right\rangle_{L^{2}}+\gamma\langle\boldsymbol{\mu},(\mathbb{D} \boldsymbol{r} \times \boldsymbol{n}) s\rangle_{L^{2}}+\gamma\left\langle\boldsymbol{\mu}, \boldsymbol{f}_{2} s\right\rangle_{L^{2}} \\
\leq & -\gamma \Gamma E \sum_{i=1}^{2} I_{i}\left[\frac{1}{2}\left(\mu_{i}^{B \Gamma}\right)^{2}-\frac{1}{6}\left(\mu_{i}^{B \Gamma}\right)^{3}+\frac{1}{12}\left(\mu_{i}^{B \Gamma}\right)^{4}\right]-\gamma \Gamma G I_{3}\left[\frac{1}{2}\left(\mu_{3}^{B \Gamma}\right)^{2}-\frac{1}{6}\left(\mu_{3}^{B \Gamma}\right)^{3}+\frac{1}{12}\left(\mu_{3}^{B \Gamma}\right)^{4}\right] \\
& +\gamma \Gamma\left(\boldsymbol{m}^{B \Gamma}\right)^{T} \boldsymbol{\mu}^{B \Gamma}-\gamma E \sum_{i=1}^{2} I_{i}\left\langle 1, \frac{1}{2} \mu_{i}^{2}-\frac{1}{3} \mu_{i}^{3}+\frac{1}{4} \mu_{i}^{4}\right\rangle_{L^{2}}-\gamma G I_{3}\left\langle 1, \frac{1}{2} \mu_{3}^{2}-\frac{1}{3} \mu_{3}^{3}+\frac{1}{4} \mu_{3}^{4}\right\rangle_{L^{2}} \\
& +\gamma \Gamma J_{0 M} \epsilon_{14}\|\boldsymbol{\mu}\|_{L^{2}}^{2}+\gamma \Gamma J_{0 M} \frac{1}{4 \epsilon_{14}}\left\langle 1, \omega_{1}^{4}+\omega_{2}^{4}+\omega_{3}^{4}\right\rangle_{L^{2}}+\gamma\langle\boldsymbol{\mu},(\mathbb{D} \boldsymbol{r} \times \boldsymbol{n}) s\rangle_{L^{2}}+\gamma\left\langle\boldsymbol{\mu}, \boldsymbol{f}_{2} s\right\rangle_{L^{2}} .
\end{aligned}
$$


Substituting (51), (54), (55), and (57) into (49) results in

$$
\begin{aligned}
\mathcal{L} U_{1} \leq & \frac{\gamma \Gamma m_{0}}{2}\left\|\tilde{\boldsymbol{r}}_{2}^{B \Gamma}\right\|^{2}+\gamma \Gamma \lambda_{M}\left(\boldsymbol{J}_{0}\right)\left\|\omega^{B \Gamma}\right\|^{2}+\gamma \Gamma \varrho\left(\boldsymbol{n}^{B \Gamma}\right)^{T} \boldsymbol{R}_{1}\left(\boldsymbol{q}^{B \Gamma}\right) \boldsymbol{\vartheta}^{B \Gamma}+\gamma \Gamma\left(\boldsymbol{m}^{B \Gamma}\right)^{T} \boldsymbol{\mu}^{B \Gamma} \\
& -\gamma \Gamma G \sum_{i=1}^{2} \bar{A}_{i}\left[\frac{1}{2}\left(\eta_{i}^{B \Gamma}\right)^{2}-\frac{1}{6}\left(\eta_{i}^{B \Gamma}\right)^{3}+\frac{1}{12}\left(\eta_{i}^{B \Gamma}\right)^{4}\right]-\gamma \Gamma E A\left[\frac{1}{2}\left(\varepsilon^{B \Gamma}\right)^{2}-\frac{1}{6}\left(\varepsilon^{B \Gamma}\right)^{3}+\frac{1}{12}\left(\varepsilon^{B \Gamma}\right)^{4}\right] \\
& -\gamma \Gamma E \sum_{i=1}^{2} I_{i}\left[\frac{1}{2}\left(\mu_{i}^{B \Gamma}\right)^{2}-\frac{1}{6}\left(\mu_{i}^{B \Gamma}\right)^{3}+\frac{1}{12}\left(\mu_{i}^{B \Gamma}\right)^{4}\right]-\gamma \Gamma G I_{3}\left[\frac{1}{2}\left(\mu_{3}^{B \Gamma}\right)^{2}-\frac{1}{6}\left(\mu_{3}^{B \Gamma}\right)^{3}+\frac{1}{12}\left(\mu_{3}^{B \Gamma}\right)^{4}\right] \\
& +\left(-\frac{\gamma m_{0}}{2}+\frac{\gamma \Gamma \Gamma_{0} \varrho}{4 \epsilon_{11}}\right)\left\|\tilde{\boldsymbol{r}}_{t}\right\|_{L^{2}}^{2}+\left(-\frac{\gamma \lambda_{m}\left(\boldsymbol{J}_{0}\right)}{2}+\gamma \Gamma m_{0} \varrho \epsilon_{11}\right)\|\omega\|_{L^{2}}^{2}+(1-\varrho) \gamma \Gamma\left(\boldsymbol{n}^{B \Gamma}\right)^{T} \boldsymbol{R}_{1}\left(\boldsymbol{q}^{B \Gamma}\right) \boldsymbol{r}^{0} \\
& -\gamma G \sum_{i=1}^{2} \bar{A}_{i}\left\langle 1, \frac{1}{2} \eta_{i}^{2}-\frac{1}{3} \eta_{i}^{3}+\frac{1}{4} \eta_{i}^{4}\right\rangle_{L^{2}}-\gamma E A\left\langle 1, \frac{1}{2} \varepsilon^{2}-\frac{1}{3} \varepsilon^{3}+\frac{1}{4} \varepsilon^{4}\right\rangle_{L^{2}} \\
& +(1-\varrho) E A\left(\bar{\epsilon}_{12} \varepsilon^{2}+\left(\bar{\epsilon}_{13}+\frac{\bar{\epsilon}_{13}^{43}}{4}\right) \varepsilon^{4}\right)+\gamma \Gamma \varrho G \sum_{i=1}^{2} \bar{A}_{i}\left\langle 1, \epsilon_{12} \eta_{i}^{2}+\left(\epsilon_{13}+\frac{1}{4} \epsilon_{13}^{\frac{4}{3}}\right) \eta_{i}^{4}\right\rangle_{L^{2}} \\
& +\gamma \Gamma \varrho\left(G \bar{A}_{1} \vee G \bar{A}_{2}\right)\left(\frac{1}{4 \epsilon_{12}}+\frac{1}{16 \epsilon_{13}}\right)\left\langle 1, \mu_{1}^{2}+\mu_{2}^{2}\right\rangle_{L^{2}}+2 \gamma \Gamma J_{0 M} \epsilon_{14}\|\boldsymbol{\mu}\|_{L^{2}}^{2}+\gamma \Gamma J_{0 M} \frac{1}{2 \epsilon_{14}}\left\langle 1, \omega_{1}^{4}+\omega_{2}^{4}+\omega_{3}^{4}\right\rangle_{L^{2}} \\
& +\gamma \Gamma \varrho\left(G \bar{A}_{1} \vee G \bar{A}_{2}\right) \frac{1}{12 \epsilon_{13}^{4}}\left\langle 1, \mu_{1}^{4}+\mu_{2}^{4}\right\rangle_{L^{2}}-\gamma E \sum_{i=1}^{2} I_{i}\left\langle 1, \frac{1}{2} \mu_{i}^{2}-\frac{1}{3} \mu_{i}^{3}+\frac{1}{4} \mu_{i}^{4}\right\rangle_{L^{2}} \\
& -\gamma G I_{3}\left\langle 1, \frac{1}{2} \mu_{3}^{2}-\frac{1}{3} \mu_{3}^{3}+\frac{1}{4} \mu_{3}^{4}\right\rangle_{L^{2}}+\gamma\left\langle\boldsymbol{\vartheta}+(1-\varrho) \mathbb{D} \boldsymbol{r}^{0}, \boldsymbol{f}_{1} s\right\rangle_{L^{2}}+\gamma\left\langle\boldsymbol{\mu}, \boldsymbol{f}_{2} s\right\rangle_{L^{2}} \\
& +(1-\varrho) E A \Gamma\left(\frac{1}{4 \bar{\epsilon}_{12}}+\frac{1}{16 \bar{\epsilon}_{13}}+\frac{1}{12 \bar{\epsilon}_{13}^{4}}\right),
\end{aligned}
$$

where we have used the fact that $-\gamma\langle\boldsymbol{n},(\boldsymbol{\mu} \times \mathbb{D} \boldsymbol{r}) s\rangle_{L^{2}}+\gamma\langle\boldsymbol{\mu},(\mathbb{D} \boldsymbol{r} \times \boldsymbol{n}) s\rangle_{L^{2}}=0$.

Calculation of $\mathcal{L} U_{B}$ : Differentiating $U_{B}$ defined in (38) along the solutions of (36) results in

$$
\begin{aligned}
\mathcal{L} U_{B}= & \left(\boldsymbol{n}^{B 0}\right)^{T} \tilde{\boldsymbol{r}}^{B 0}+\left(\tilde{\boldsymbol{r}}^{B 0}\right)^{T} \boldsymbol{f}_{1}^{B 0}+\left(\boldsymbol{m}^{B 0}\right)^{T} \boldsymbol{\omega}^{B 0}+\left(\boldsymbol{m}^{B 0}\right)^{T} \boldsymbol{f}_{2}^{B 0}+\left(\gamma_{1} \frac{\tilde{\boldsymbol{r}}_{1}^{B \Gamma}}{\Delta^{B \Gamma}}+\tilde{\boldsymbol{r}}_{2}^{B \Gamma}+\gamma \Gamma \boldsymbol{R}_{1}\left(\boldsymbol{q}^{B \Gamma}\right) \boldsymbol{\vartheta}^{B \Gamma}\right)^{T} \\
& \times\left[\gamma_{1} \boldsymbol{M}_{H} \frac{\tilde{\boldsymbol{r}}_{2}^{B \Gamma} \Delta^{B \Gamma}-\tilde{\boldsymbol{r}}_{1} \Delta^{B \Gamma}}{\left(\Delta^{B \Gamma}\right)^{2}}-\boldsymbol{n}^{B \Gamma}+\boldsymbol{\phi}_{1 B}+\boldsymbol{f}_{1}^{B \Gamma}+\gamma \Gamma \boldsymbol{R}_{1}\left(\boldsymbol{q}^{B \Gamma}\right) \boldsymbol{\vartheta}^{B \Gamma}+\gamma \Gamma \dot{\boldsymbol{R}}_{1}\left(\boldsymbol{q}^{B \Gamma}\right) \boldsymbol{\vartheta}^{B \Gamma}\right]+2 \gamma_{1} \frac{\left(\tilde{\boldsymbol{r}}_{1}^{B \Gamma}\right)^{T} \boldsymbol{K}_{1 B} \tilde{r}_{2}^{B \Gamma}}{\Delta^{B \Gamma}} \\
& +\left(\gamma_{2} \boldsymbol{K}^{T}\left(\boldsymbol{q}^{B \Gamma}\right) \operatorname{col}\left(q_{1}^{B \Gamma}-1, \overline{\boldsymbol{q}}^{B \Gamma}\right)+\boldsymbol{\omega}^{B \Gamma}+\gamma \Gamma \boldsymbol{\mu}^{B \Gamma}\right)^{T}\left[\frac{\gamma_{2}}{4} \boldsymbol{J}_{H} \boldsymbol{\omega}^{B \Gamma}+\gamma_{2} \dot{\boldsymbol{K}}^{T}\left(\boldsymbol{q}^{B \Gamma}\right) \operatorname{col}\left(q_{1}^{B \Gamma}-1, \overline{\boldsymbol{q}}^{B \Gamma}\right)-\boldsymbol{m}^{B \Gamma}\right. \\
& \left.+\boldsymbol{\phi}_{2 B}+\boldsymbol{f}_{2}^{B \Gamma}+\gamma \Gamma \dot{\boldsymbol{\mu}}^{B \Gamma}\right]+2 \gamma_{2} k_{2 B} \operatorname{col}\left(q_{1}^{B \Gamma}-1, \overline{\boldsymbol{q}}^{B \Gamma}\right)^{T} \boldsymbol{K}\left(\boldsymbol{q}^{B \Gamma}\right) \boldsymbol{\omega}^{B \Gamma} .
\end{aligned}
$$

Substituting (48), (58), and (59) into (45) results in

$$
\mathcal{L} U \leq \Omega+\Omega_{B}+(1-\varrho) E A \Gamma\left(\frac{1}{4 \bar{\epsilon}_{12}}+\frac{1}{16 \bar{\epsilon}_{13}}+\frac{1}{12 \bar{\epsilon}_{13}^{4}}\right)
$$

where

$$
\begin{aligned}
\Omega=( & \left.-\frac{\gamma m_{0}}{2}+\frac{\gamma \Gamma m_{0} \varrho}{4 \epsilon_{11}}\right)\left\|\tilde{\boldsymbol{r}}_{2}\right\|_{L^{2}}^{2}+\left(-\frac{\gamma \lambda_{m}\left(\boldsymbol{J}_{0}\right)}{2}+\gamma \Gamma m_{0} \varrho \epsilon_{11}\right)\|\omega\|_{L^{2}}^{2}-\gamma G \sum_{i=1}^{2} \bar{A}_{i}\left\langle 1, \frac{1}{2} \eta_{i}^{2}-\frac{1}{3} \eta_{i}^{3}+\frac{1}{4} \eta_{i}^{4}\right\rangle_{L^{2}} \\
& -\gamma E A\left\langle 1, \frac{1}{2} \varepsilon^{2}-\frac{1}{3} \varepsilon^{3}+\frac{1}{4} \varepsilon^{4}\right\rangle_{L^{2}}+(1-\varrho) E A\left(\bar{\epsilon}_{12} \varepsilon^{2}+\left(\bar{\epsilon}_{13}+\frac{\bar{\epsilon}_{13}^{4 / 3}}{4}\right) \varepsilon^{4}\right) \\
& +\gamma \Gamma \varrho G \sum_{i=1}^{2} \overline{A_{i}}\left\langle 1, \epsilon_{12} \eta_{i}^{2}+\left(\epsilon_{13}+\frac{1}{4} \epsilon_{13}^{3}\right) \eta_{i}^{4}\right\rangle_{L^{2}}+\gamma \Gamma \varrho\left(G \bar{A}_{1} \vee G \bar{A}_{2}\right)\left(\frac{1}{4 \epsilon_{12}}+\frac{1}{16 \epsilon_{13}}\right)\left\langle 1, \mu_{1}^{2}+\mu_{2}^{2}\right\rangle_{L^{2}} \\
& +2 \gamma \Gamma J_{0 M} \epsilon_{14}\|\boldsymbol{\mu}\|_{L^{2}}^{2}+\gamma \Gamma J_{0 M} \frac{1}{2 \epsilon_{14}}\left\langle 1, \omega_{1}^{4}+\omega_{2}^{4}+\omega_{3}^{4}\right\rangle_{L^{2}}+\gamma \Gamma \varrho\left(G \bar{A}_{1} \vee G \bar{A}_{2}\right) \frac{1}{12 \epsilon_{13}^{4}}\left\langle 1, \mu_{1}^{4}+\mu_{2}^{4}\right\rangle_{L^{2}} \\
& -\gamma E \sum_{i=1}^{2} I_{i}\left\langle 1, \frac{1}{2} \mu_{i}^{2}-\frac{1}{3} \mu_{i}^{3}+\frac{1}{4} \mu_{i}^{4}\right\rangle_{L^{2}}-\gamma G I_{3}\left\langle 1, \frac{1}{2} \mu_{3}^{2}-\frac{1}{3} \mu_{3}^{3}+\frac{1}{4} \mu_{3}^{4}\right\rangle_{L^{2}}+\left\langle\boldsymbol{v}, \boldsymbol{f}_{1}\right\rangle_{L^{2}}+\left\langle\omega, \boldsymbol{f}_{2}\right\rangle_{L^{2}} \\
& +\gamma\left\langle\boldsymbol{\vartheta}+(1-\varrho) \mathbb{D} \boldsymbol{r}^{0}, \boldsymbol{f}_{1} s\right\rangle_{L^{2}}+\gamma\left\langle\boldsymbol{\mu}, \boldsymbol{f}_{2} s\right\rangle_{L^{2}}
\end{aligned}
$$

and

$$
\begin{aligned}
& \Omega_{B}=-\gamma \Gamma G \sum_{i=1}^{2} \bar{A}_{i}\left[\frac{1}{2}\left(\eta_{i}^{B \Gamma}\right)^{2}-\frac{1}{6}\left(\eta_{i}^{B \Gamma}\right)^{3}+\frac{1}{12}\left(\eta_{i}^{B \Gamma}\right)^{4}\right]-\gamma \Gamma E A\left[\frac{1}{2}\left(\varepsilon^{B \Gamma}\right)^{2}-\frac{1}{6}\left(\varepsilon^{B \Gamma}\right)^{3}+\frac{1}{12}\left(\varepsilon^{B \Gamma}\right)^{4}\right] \\
& -\gamma \Gamma E \sum_{i=1}^{2} I_{i}\left[\frac{1}{2}\left(\mu_{i}^{B \Gamma}\right)^{2}-\frac{1}{6}\left(\mu_{i}^{B \Gamma}\right)^{3}+\frac{1}{12}\left(\mu_{i}^{B \Gamma}\right)^{4}\right]-\gamma \Gamma G I_{3}\left[\frac{1}{2}\left(\mu_{3}^{B \Gamma}\right)^{2}-\frac{1}{6}\left(\mu_{3}^{B \Gamma}\right)^{3}+\frac{1}{12}\left(\mu_{3}^{B \Gamma}\right)^{4}\right] \\
& +\frac{\gamma \Gamma m_{0}}{2}\left\|\tilde{\boldsymbol{r}}_{2}^{B \Gamma}\right\|^{2}+\gamma \Gamma \lambda_{M}\left(\boldsymbol{J}_{0}\right)\left\|\omega^{B \Gamma}\right\|^{2}+\left(\tilde{\boldsymbol{r}}^{B 0}\right)^{T} \boldsymbol{f}_{1}^{B 0}+\left(\boldsymbol{\omega}^{B 0}\right)^{T} \boldsymbol{f}_{2}^{B 0} \\
& +\left(\gamma_{1} \frac{\tilde{\boldsymbol{r}}_{1}^{B \Gamma}}{\Delta^{B \Gamma}}+\tilde{\boldsymbol{r}}_{2}^{B \Gamma}+\gamma \Gamma \boldsymbol{R}_{1}\left(\boldsymbol{q}^{B \Gamma}\right) \boldsymbol{\vartheta}^{B \Gamma}\right)^{T}\left[\gamma_{1} \boldsymbol{M}_{H} \frac{\tilde{r}_{2}^{B \Gamma} \Delta^{B \Gamma}-\tilde{\boldsymbol{r}}_{1} \dot{\Delta}^{B \Gamma}}{\left(\Delta^{B \Gamma}\right)^{2}}+\boldsymbol{\phi}_{1 B}+\boldsymbol{f}_{1}^{B \Gamma}+\gamma \Gamma \boldsymbol{R}_{1}\left(\boldsymbol{q}^{B \Gamma}\right) \dot{\boldsymbol{\vartheta}}^{B \Gamma}+\gamma \Gamma \dot{\boldsymbol{R}}_{1}\left(\boldsymbol{q}^{B \Gamma}\right) \boldsymbol{\vartheta}^{B \Gamma}\right] \\
& +(1-\varrho) \gamma \Gamma\left(\boldsymbol{n}^{B \Gamma}\right)^{T} \boldsymbol{R}_{1}\left(\boldsymbol{q}^{B \Gamma}\right) \boldsymbol{r}^{0}+2 \gamma_{1} \frac{\left(\tilde{\boldsymbol{r}}_{1}^{B \Gamma}\right)^{T} \boldsymbol{K}_{1 B} \tilde{\boldsymbol{r}}_{2}^{B \Gamma}}{\Delta^{B \Gamma}}+\gamma_{1} \frac{\left(\tilde{\boldsymbol{r}}_{1}^{B \Gamma}\right)^{T} \boldsymbol{n}^{B \Gamma}}{\Delta^{B \Gamma}} \\
& +\left(\gamma_{2} \boldsymbol{K}^{T}\left(\boldsymbol{q}^{B \Gamma}\right) \operatorname{col}\left(q_{1}^{B \Gamma}-1, \overline{\boldsymbol{q}}^{B \Gamma}\right)+\boldsymbol{\omega}^{B \Gamma}+\gamma \Gamma \boldsymbol{\mu}^{B \Gamma}\right)^{T}\left[\frac{\gamma_{2}}{4} \boldsymbol{J}_{H} \boldsymbol{\omega}^{B \Gamma}+\gamma_{2} \dot{\boldsymbol{K}}^{T}\left(\boldsymbol{q}^{B \Gamma}\right) \operatorname{col}\left(q_{1}^{B \Gamma}-1, \overline{\boldsymbol{q}}^{B \Gamma}\right)\right. \\
& \left.+\boldsymbol{\phi}_{2 B}+\boldsymbol{f}_{2}^{B \Gamma}+\gamma \Gamma \dot{\boldsymbol{\mu}}^{B \Gamma}\right]+2 \gamma_{2} k_{2 B} \operatorname{col}\left(q_{1}^{B \Gamma}-1, \overline{\boldsymbol{q}}^{B \Gamma}\right)^{T} \boldsymbol{K}\left(\boldsymbol{q}^{B \Gamma}\right) \boldsymbol{\omega}^{B \Gamma}+\gamma_{2}\left(\boldsymbol{K}^{T}\left(\boldsymbol{q}^{B \Gamma}\right) \operatorname{col}\left(q_{1}^{B \Gamma}-1, \overline{\boldsymbol{q}}^{B \Gamma}\right)\right)^{T} \boldsymbol{m}^{B \Gamma},
\end{aligned}
$$


and we have subtracted and added the terms $\gamma_{1} \frac{\left(\tilde{r}_{1}^{B \Gamma}\right)^{T} n^{B \Gamma}}{\Delta^{B \Gamma}}$ and $\gamma_{2}\left(\boldsymbol{K}^{T}\left(\boldsymbol{q}^{B \Gamma}\right) \operatorname{col}\left(q_{1}^{B \Gamma}-1, \overline{\boldsymbol{q}}^{B \Gamma}\right)\right)^{T} \boldsymbol{m}^{B \Gamma}$ to $\Omega_{B}$. From (62) and (16), we choose the boundary control vectors $\phi_{1 B}$ and $\phi_{2 B}$ as follows:

$$
\begin{aligned}
\boldsymbol{\phi}_{1 B}= & -\left(\boldsymbol{K}_{1 B}+\boldsymbol{I}_{3}\left(\epsilon_{11 B}+\epsilon_{12 B}\right)\right)\left(\gamma_{1} \frac{\tilde{r}_{1}^{B \Gamma}}{\Delta^{B \Gamma}}+\tilde{\boldsymbol{r}}_{2}^{B \Gamma}+\gamma \Gamma \boldsymbol{R}_{1}\left(\boldsymbol{q}^{B \Gamma}\right) \boldsymbol{\vartheta}^{B \Gamma}\right)-\gamma_{1} \boldsymbol{M}_{H} \frac{\tilde{r}_{2}^{B \Gamma} \Delta^{B \Gamma}-\tilde{\boldsymbol{r}}_{1}^{B \Gamma} \dot{\Delta}^{B \Gamma}}{\left(\Delta^{B \Gamma}\right)^{2}}+\boldsymbol{D}_{11}^{\Gamma} \tilde{\boldsymbol{r}}_{2}^{B \Gamma} \\
& +\boldsymbol{D}_{12}^{\Gamma}\left(\tilde{\boldsymbol{r}}_{2}^{B \Gamma} \otimes \tilde{\boldsymbol{r}}_{2}^{B \Gamma}\right) \tilde{\boldsymbol{r}}_{2}^{B \Gamma}-\gamma \Gamma \boldsymbol{R}_{1}\left(\boldsymbol{q}^{B \Gamma}\right) \dot{\boldsymbol{\vartheta}}^{B \Gamma}-\gamma \Gamma \dot{\boldsymbol{R}}_{1}\left(\boldsymbol{q}^{B \Gamma}\right) \boldsymbol{\vartheta}^{B \Gamma}, \\
\boldsymbol{\phi}_{2 B}= & -\left(k_{2 B}+\epsilon_{21 B}+\epsilon_{22 B}\right)\left(\gamma_{2} \boldsymbol{K}^{T}\left(\boldsymbol{q}^{B \Gamma}\right) \operatorname{col}\left(q_{1}^{B \Gamma}-1, \overline{\boldsymbol{q}}^{B \Gamma}\right)+\boldsymbol{\omega}^{B \Gamma}+\gamma \Gamma \boldsymbol{\mu}^{B \Gamma}\right)-\frac{\gamma_{2}}{4} \boldsymbol{J}_{H} \boldsymbol{\omega}^{B \Gamma} \\
& -\gamma_{2} \dot{\boldsymbol{K}}^{T}\left(\boldsymbol{q}^{B \Gamma}\right) \operatorname{col}\left(q_{1}^{B \Gamma}-1, \overline{\boldsymbol{q}}^{B \Gamma}\right)+\boldsymbol{D}_{21}^{\Gamma} \boldsymbol{\omega}^{B \Gamma}+\boldsymbol{D}_{22}^{\Gamma}\left(\boldsymbol{\omega} \otimes \boldsymbol{\omega}^{B \Gamma}\right) \boldsymbol{\omega}^{B \Gamma}-\gamma \Gamma \dot{\boldsymbol{\mu}}^{B \Gamma},
\end{aligned}
$$

where $\epsilon_{i j B},(i, j)=1,2$ are positive constants, and $\boldsymbol{I}_{3}$ is the $3 \times 3$ identity matrix.

Remark 5.2. The boundary control vectors $\phi_{1 B}$ and $\phi_{2 B}$ only measurements from the actuated end of the beam with a note that $\dot{\Delta}^{B \Gamma}=\frac{\left(\tilde{\boldsymbol{r}}_{1}^{B \Gamma}\right)^{T} \boldsymbol{K}_{1 B} \tilde{r}_{2}^{B \Gamma}}{\Delta^{B \Gamma}}$ and $\dot{\boldsymbol{R}}_{1}\left(\boldsymbol{q}^{B \Gamma}\right)=\frac{\partial \boldsymbol{R}_{1}\left(\boldsymbol{q}^{B \Gamma}\right)}{\partial \boldsymbol{q}^{B \Gamma}} \boldsymbol{K}\left(\boldsymbol{q}^{B \Gamma}\right) \boldsymbol{\omega}^{B \Gamma}$. The terms $\tilde{\boldsymbol{r}}_{1}^{B \Gamma}$ and $q^{B \Gamma}$ can be measured by using translational and rotational displacement sensors $\left(q^{B \Gamma}\right.$ is determined from Euler angles via the relationship (8). The terms $\boldsymbol{\vartheta}^{B \Gamma}$ and $\boldsymbol{\mu}^{B \Gamma}$ can be measured by using strain gauges. The terms $\tilde{\boldsymbol{r}}_{2}^{B \Gamma}, \omega^{B \Gamma}, \dot{\boldsymbol{\vartheta}}^{B \Gamma}$, and $\dot{\boldsymbol{\mu}}^{B \Gamma}$ can be obtained by using band-pass filters, through which the measurements of $\tilde{\boldsymbol{r}}_{1}^{B \Gamma}, \boldsymbol{q}^{B \Gamma}, \boldsymbol{\vartheta}^{B \Gamma}$, and $\boldsymbol{\mu}^{B \Gamma}$ are passed, respectively. Using this method, sensors are made commercially (e.g., [41]).

Substituting (63) into (62) gives

$$
\begin{aligned}
\Omega_{B}= & -\gamma \Gamma G \sum_{i=1}^{2} \bar{A}_{i}\left[\frac{1}{2}\left(\eta_{i}^{B \Gamma}\right)^{2}-\frac{1}{6}\left(\eta_{i}^{B \Gamma}\right)^{3}+\frac{1}{12}\left(\eta_{i}^{B \Gamma}\right)^{4}\right]-\gamma \Gamma E A\left[\frac{1}{2}\left(\varepsilon^{B \Gamma}\right)^{2}-\frac{1}{6}\left(\varepsilon^{B \Gamma}\right)^{3}+\frac{1}{12}\left(\varepsilon^{B \Gamma}\right)^{4}\right] \\
& -\gamma \Gamma E \sum_{i=1}^{2} I_{i}\left[\frac{1}{2}\left(\mu_{i}^{B \Gamma}\right)^{2}-\frac{1}{6}\left(\mu_{i}^{B \Gamma}\right)^{3}+\frac{1}{12}\left(\mu_{i}^{B \Gamma}\right)^{4}\right]-\gamma \Gamma G I_{3}\left[\frac{1}{2}\left(\mu_{3}^{B \Gamma}\right)^{2}-\frac{1}{6}\left(\mu_{3}^{B \Gamma}\right)^{3}+\frac{1}{12}\left(\mu_{3}^{B \Gamma}\right)^{4}\right] \\
& +\frac{\gamma \Gamma m_{0}}{2}\left\|\tilde{\boldsymbol{r}}_{2}^{B \Gamma}\right\|^{2}+\gamma \Gamma \lambda_{M}\left(\boldsymbol{J}_{0}\right)\left\|\boldsymbol{\omega}^{B \Gamma}\right\|^{2}+\left(\tilde{\boldsymbol{r}}^{B 0}\right)^{T} \boldsymbol{f}_{1}^{B 0}+\left(\boldsymbol{\omega}^{B 0}\right)^{T} \boldsymbol{f}_{2}^{B 0} \\
& -\left(\gamma_{1} \frac{\tilde{\boldsymbol{r}}_{1}^{B \Gamma}}{\Delta^{B \Gamma}}+\tilde{\boldsymbol{r}}_{2}^{B \Gamma}+\gamma \Gamma \boldsymbol{R}_{1}\left(\boldsymbol{q}^{B \Gamma}\right) \boldsymbol{\vartheta}^{B \Gamma}\right)^{T}\left(\boldsymbol{K}_{1 B}+\boldsymbol{I}_{3}\left(\epsilon_{11 B}+\epsilon_{12 B}\right)\right)\left(\gamma_{1} \frac{\tilde{\boldsymbol{r}}_{1}^{B \Gamma}}{\Delta^{B \Gamma}}+\tilde{\boldsymbol{r}}_{2}^{B \Gamma}+\gamma \Gamma \boldsymbol{R}_{1}\left(\boldsymbol{q}^{B \Gamma}\right) \boldsymbol{\vartheta}^{B \Gamma}\right) \\
& +\left(\gamma_{1} \frac{\tilde{\boldsymbol{r}}_{1}^{B \Gamma}}{\Delta^{B \Gamma}}+\tilde{\boldsymbol{r}}_{2}^{B \Gamma}+\gamma \Gamma \boldsymbol{R}_{1}\left(\boldsymbol{q}^{B \Gamma}\right) \boldsymbol{\vartheta}^{B \Gamma}\right)^{T} \boldsymbol{f}_{10}^{B \Gamma}+2 \gamma_{1} \frac{\left.\tilde{\boldsymbol{r}}_{1}^{B \Gamma}\right)^{T} \boldsymbol{K}_{1 B} \tilde{\boldsymbol{r}}_{2}^{B \Gamma}}{\Delta^{B \Gamma}}+\gamma_{1} \frac{\left(\tilde{r}_{1}^{\Gamma \Gamma}\right)^{T} \boldsymbol{n}^{B \Gamma}}{\Delta^{B \Gamma}}+(1-\varrho) \gamma \Gamma\left(\boldsymbol{n}^{B \Gamma}\right)^{T} \boldsymbol{R}_{1}\left(\boldsymbol{q}^{B \Gamma}\right) \boldsymbol{r}^{0} \\
& -\left(k_{2 B}+\epsilon_{21 B}+\epsilon_{22 B}\right)\left\|\gamma_{2} \boldsymbol{K}^{T}\left(\boldsymbol{q}^{B \Gamma}\right) \operatorname{col}\left(q_{1}^{B \Gamma}-1, \overline{\boldsymbol{q}}^{B \Gamma}\right)+\boldsymbol{\omega}^{B \Gamma}+\gamma \Gamma \boldsymbol{\mu}^{B \Gamma}\right\|^{2} \\
& +\left(\gamma_{2} \boldsymbol{K}^{T}\left(\boldsymbol{q}^{B \Gamma}\right) \operatorname{col}\left(q_{1}^{B \Gamma}-1, \overline{\boldsymbol{q}}^{B \Gamma}\right)+\boldsymbol{\omega}^{B \Gamma}+\gamma \Gamma \boldsymbol{\mu}^{B \Gamma}\right)^{T} \boldsymbol{f}_{20}^{B \Gamma}+2 \gamma_{2} k_{2 B} \operatorname{col}\left(q_{1}^{B \Gamma}-1, \overline{\boldsymbol{q}}^{B \Gamma}\right)^{T} \boldsymbol{K}\left(\boldsymbol{q}^{B \Gamma}\right) \boldsymbol{\omega}^{B \Gamma} \\
& +\gamma_{2}\left(\boldsymbol{K}^{T}\left(\boldsymbol{q}^{B \Gamma}\right) \operatorname{col}\left(q_{1}^{B \Gamma}-1, \overline{\boldsymbol{q}}^{B \Gamma}\right)\right)^{T} \boldsymbol{m}^{B \Gamma} .
\end{aligned}
$$

We now need the following inequalities to calculate the bounds of $\Omega$ and $\Omega_{B}$ :

$$
\begin{aligned}
& -\left(\frac{1}{2} \eta^{2}-\frac{1}{3} \eta^{3}+\frac{1}{4} \eta^{4}\right) \leq-\frac{1}{6}\left(\eta^{2}+\eta^{4}\right), \\
& -\left(\frac{1}{2} \eta^{2}-\frac{1}{6} \eta^{3}+\frac{1}{12} \eta^{4}\right) \leq-\frac{5}{12} \eta^{2}-\frac{1}{24} \eta^{4},
\end{aligned}
$$

for all $\eta \in \mathbb{R}$. Applying the inequalities (65) and Young's inequality to $\Omega$ with $f_{1}$ and $f_{2}$ given in (6) results in

$$
\begin{aligned}
\Omega \leq & -k_{11}\|\boldsymbol{v}\|_{L^{2}}^{2}-k_{11}^{N}\left\langle 1, v_{1}^{4}+v_{2}^{4}+v_{3}^{4}\right\rangle_{L^{2}}-k_{12}\|\omega\|_{L^{2}}^{2}-k_{12}^{N}\left\langle 1, \omega_{1}^{4}+\omega_{2}^{4}+\omega_{3}^{4}\right\rangle_{L^{2}} \\
& -k_{21}\|\boldsymbol{\vartheta}\|_{L^{2}}^{2}-k_{21}^{N}\left\langle 1, \eta_{1}^{4}+\eta_{2}^{4}+\varepsilon^{4}\right\rangle_{L^{2}}-k_{22}\|\boldsymbol{\mu}\|_{L^{2}}^{2}-k_{22}^{N}\left\langle 1, \mu_{1}^{4}+\mu_{2}^{4}+\mu^{4}\right\rangle_{L^{2}} \\
& +\left(\frac{1}{4 \epsilon_{10}}+\frac{\gamma \Gamma}{4 \epsilon_{140}}+\frac{\gamma(1-\varrho) \Gamma}{4 \bar{\epsilon}_{130}}\right)\left\|\boldsymbol{f}_{10}\right\|_{L^{2}}^{2}+\left(\frac{1}{4 \epsilon_{210}}+\frac{\gamma \Gamma}{4 \epsilon_{240}}\right)\left\|\boldsymbol{f}_{20}\right\|_{L^{2}}^{2}+\gamma \Gamma(1-\varrho)\left(\frac{\lambda_{M}\left(\boldsymbol{D}_{11}\right)}{4 \bar{\epsilon}_{110}}+\frac{\lambda_{M}\left(\boldsymbol{D}_{12}\right)}{4 \bar{\epsilon}_{120}^{4}}+\bar{\epsilon}_{130} \Gamma\right),
\end{aligned}
$$


where we have used $\left\|\tilde{\boldsymbol{r}}_{2}\right\|_{L^{2}}^{2}=\|\boldsymbol{v}\|_{L^{2}}^{2} ;\left(v_{1}, v_{2}, v_{3}\right)$ and $\left(\omega_{1}, \omega_{2}, \omega_{3}\right)$ are components of $\boldsymbol{v}$ and $\boldsymbol{\omega}$, respectively, and

$$
\begin{aligned}
k_{11}= & \lambda_{m}\left(\boldsymbol{D}_{11}\right)+\frac{\gamma m_{0}}{2}-\frac{\gamma \Gamma m_{0} \varrho}{4 \epsilon_{11}}-\epsilon_{110}-\gamma \Gamma \lambda_{M}\left(\boldsymbol{D}_{11}\right) \epsilon_{120}-\gamma \Gamma(1-\varrho) \lambda_{M}\left(\boldsymbol{D}_{11}\right) \bar{\epsilon}_{120}, \\
k_{11}^{N}= & \lambda_{m}\left(\boldsymbol{D}_{12}\right)-\frac{3}{4} \gamma \Gamma \epsilon_{130}^{\frac{4}{3}} \lambda_{M}\left(\boldsymbol{D}_{12}\right)-\frac{3}{4} \gamma \Gamma(1-\varrho) \bar{\epsilon}_{110}^{\frac{4}{3}} \lambda_{M}\left(\boldsymbol{D}_{12}\right), \\
k_{12}= & \lambda_{m}\left(\boldsymbol{D}_{21}\right)+\frac{\gamma \lambda_{m}\left(\boldsymbol{J}_{0}\right)}{2}-\gamma \Gamma m_{0} \varrho \epsilon_{11}-\epsilon_{210}-\gamma \Gamma \lambda_{M}\left(\boldsymbol{D}_{21}\right) \epsilon_{220}, \\
k_{12}^{N}= & \lambda_{m}\left(\boldsymbol{D}_{22}\right)-\frac{3}{4} \gamma \Gamma \epsilon_{230}^{\frac{3}{4}} \lambda_{M}\left(\boldsymbol{D}_{22}\right), \\
k_{21}= & \gamma\left[\left(\frac{1}{6}\left(G \bar{A}_{1} \wedge G \bar{A}_{2}\right)-\epsilon_{12} \varrho \Gamma\left(G \bar{A}_{1} \vee G \bar{A}_{2}\right)\right) \wedge\left(\frac{1}{6}-(1-\varrho) \bar{\epsilon}_{12}\right) E A-\epsilon_{140} \Gamma-\frac{1}{4 \epsilon_{120}} \Gamma \lambda_{M}\left(\boldsymbol{D}_{11}\right)\right], \\
k_{21}^{N}= & \gamma\left[\left(\frac{1}{6}\left(G \bar{A}_{1} \wedge G \bar{A}_{2}\right)-\Gamma \varrho\left(\epsilon_{13}+\frac{1}{4} \epsilon_{13}^{\frac{4}{3}}\right)\left(G \bar{A}_{1} \vee G \bar{A}_{2}\right)\right) \wedge\left(\frac{1}{6}-(1-\varrho)\left(\bar{\epsilon}_{13}+\frac{\bar{\epsilon}_{13}^{4 / 3}}{4}\right)\right) E A-\frac{1}{4 \epsilon_{130}^{4}} \Gamma \lambda_{M}\left(\boldsymbol{D}_{12}\right)\right], \\
k_{22}= & \gamma\left[\left(\frac{1}{6}\left(E I_{1} \wedge E I_{2}\right)-\epsilon_{12} \varrho \Gamma\left(E I_{1} \vee E I_{2}\right)-\Gamma \varrho\left(G \bar{A}_{1} \vee G \bar{A}_{2}\right)\left(\frac{1}{4 \epsilon_{12}}+\frac{1}{16 \epsilon_{13}}\right)\right) \wedge \frac{1}{6} G I_{3}-2 \Gamma J_{0 M} \epsilon_{14}\right. \\
& \left.-\Gamma \epsilon_{240}-\frac{1}{4 \epsilon_{220}} \lambda_{M}\left(\boldsymbol{D}_{21}\right)\right], \\
k_{22}^{N}= & \gamma\left[\left(\frac{1}{6}\left(E I_{1} \wedge E I_{2}\right)-\Gamma \varrho\left(\epsilon_{13}+\frac{1}{4} \epsilon_{13}^{\frac{4}{3}}\right)\left(E I_{1} \vee E I_{2}\right)-\frac{\Gamma \varrho}{12 \epsilon_{13}^{4}}\left(G \bar{A}_{1} \vee G \bar{A}_{2}\right)\right) \wedge \frac{1}{6} G I_{3}-\frac{\Gamma}{4 \epsilon_{230}^{4}} \lambda_{M}\left(\boldsymbol{D}_{22}\right)\right],
\end{aligned}
$$

with $\epsilon_{i j 0}, i=1,2 ; j=1, \ldots, 4$ are positive constants to be chosen. Applying the inequalities (65) and Young's inequality to $\Omega_{B}$ with $\boldsymbol{f}_{1}^{B 0}$ and $\boldsymbol{f}_{2}^{B 0}$ given in (16) results in

$$
\begin{aligned}
\Omega_{B} \leq & -\left(\lambda_{m}\left(\boldsymbol{D}_{11}^{0}\right)-\epsilon_{310}\right)\left\|\tilde{\boldsymbol{r}}_{2}^{B 0}\right\|^{2}-\left(\lambda_{m}\left(\boldsymbol{D}_{21}^{0}\right)-\epsilon_{320}\right)\left\|\boldsymbol{\omega}^{B 0}\right\|^{2}-\epsilon_{11 B}\left\|\left(\gamma_{1} \frac{\tilde{\boldsymbol{r}}_{1}^{B \Gamma}}{\Delta^{B \Gamma}}+\tilde{\boldsymbol{r}}_{2}^{B \Gamma}+\gamma \Gamma \boldsymbol{R}_{1}\left(\boldsymbol{q}^{B \Gamma}\right) \boldsymbol{\vartheta}^{B \Gamma}\right)\right\|^{2} \\
& -\epsilon_{21 B}\left\|\gamma_{2} \boldsymbol{K}^{T}\left(\boldsymbol{q}^{B \Gamma}\right) \operatorname{col}\left(q_{1}^{B \Gamma}-1, \overline{\boldsymbol{q}}^{B \Gamma}\right)+\boldsymbol{\omega}^{B \Gamma}+\gamma \Gamma \boldsymbol{\mu}^{B \Gamma}\right\|^{2}+\frac{1}{4 \epsilon_{310}}\left\|\boldsymbol{f}_{10}^{B 0}\right\|^{2}+\frac{1}{4 \epsilon_{320}}\left\|\boldsymbol{f}_{20}^{B 0}\right\|^{2}+\frac{1}{4 \epsilon_{12 B}}\left\|\boldsymbol{f}_{10}^{B \Gamma}\right\|^{2} \\
& +\frac{1}{4 \epsilon_{22 B}}\left\|\boldsymbol{f}_{20}^{B \Gamma}\right\|^{2}+\Phi_{B},
\end{aligned}
$$

where

$$
\begin{aligned}
\Phi_{B} \leq & -\frac{5 \gamma \Gamma}{12}\left(G \bar{A}_{1} \wedge G \bar{A}_{2} \wedge E A\right)\left\|\boldsymbol{\vartheta}^{B \Gamma}\right\|^{2}-\frac{\gamma \Gamma}{24}\left(G \bar{A}_{1} \wedge G \bar{A}_{2} \wedge E A\right)\left(\eta_{1}^{4}+\eta_{2}^{4}+\varepsilon^{4}\right)-\frac{5 \gamma \Gamma}{12}\left(E I_{1} \wedge E I_{2} \wedge G I_{3}\right)\left\|\boldsymbol{\mu}^{B \Gamma}\right\|^{2} \\
& -\frac{\gamma \Gamma}{24}\left(E I_{1} \wedge E I_{2} \wedge G I_{3}\right)\left(\mu_{1}^{4}+\mu_{2}^{4}+\mu_{3}^{4}\right)+\frac{\gamma \Gamma m_{0}}{2}\left\|\tilde{\boldsymbol{r}}_{2}^{B \Gamma}\right\|^{2}+\gamma \Gamma \lambda_{M}\left(\boldsymbol{J}_{0}\right)\left\|\boldsymbol{\omega}^{B \Gamma}\right\|^{2}+2 \gamma_{1} \frac{\left(\tilde{\boldsymbol{r}}_{1}^{B \Gamma}\right)^{T} \boldsymbol{K}_{1 B} \tilde{\boldsymbol{r}}_{2}^{B \Gamma}}{\Delta^{B \Gamma}} \\
& -\left(\gamma_{1} \frac{\tilde{\boldsymbol{r}}_{1}^{B \Gamma}}{\Delta^{B \Gamma}}+\tilde{\boldsymbol{r}}_{2}^{B \Gamma}+\gamma \Gamma \boldsymbol{R}_{1}\left(\boldsymbol{q}^{B \Gamma}\right) \boldsymbol{\vartheta}^{B \Gamma}\right)^{T} \boldsymbol{K}_{1 B}\left(\gamma_{1} \frac{\tilde{\boldsymbol{r}}_{1}^{B \Gamma}}{\Delta^{B \Gamma}}+\tilde{\boldsymbol{r}}_{2}^{B \Gamma}+\gamma \Gamma \boldsymbol{R}_{1}\left(\boldsymbol{q}^{B \Gamma}\right) \boldsymbol{\vartheta}^{B \Gamma}\right)+\gamma_{1} \frac{\left(\tilde{\boldsymbol{r}}_{1}^{B \Gamma}\right)^{T} \boldsymbol{n}^{B \Gamma}}{\Delta^{B \Gamma}} \\
& -k_{2 B}\left\|\gamma_{2} \boldsymbol{K}^{T}\left(\boldsymbol{q}^{B \Gamma}\right) \operatorname{col}\left(q_{1}^{B \Gamma}-1, \overline{\boldsymbol{q}}^{B \Gamma}\right)+\boldsymbol{\omega}^{B \Gamma}+\gamma \Gamma \boldsymbol{\mu}^{B \Gamma}\right\|^{2}+2 \gamma_{2} k_{2 B} \operatorname{col}\left(q_{1}^{B \Gamma}-1, \overline{\boldsymbol{q}}^{B \Gamma}\right)^{T} \boldsymbol{K}\left(\boldsymbol{q}^{B \Gamma}\right) \boldsymbol{\omega}^{B \Gamma} \\
& +\gamma_{2}\left(\boldsymbol{K}^{T}\left(\boldsymbol{q}^{B \Gamma}\right) \operatorname{col}\left(q_{1}^{B \Gamma}-1, \overline{\boldsymbol{q}}^{B \Gamma}\right)\right)^{T} \boldsymbol{m}^{B \Gamma}+(1-\varrho) \gamma \Gamma\left(\boldsymbol{n}^{B \Gamma}\right)^{T} \boldsymbol{R}_{1}\left(\boldsymbol{q}^{B \Gamma}\right) \boldsymbol{r}^{0} .
\end{aligned}
$$

Expanding the appropriate terms and using the expression of $\boldsymbol{n}^{B \Gamma}$ and $\boldsymbol{m}^{B \Gamma}$ given in (12) and (13), we can further calculate the upper-bound of $\Phi_{B}$ as follows

$$
\begin{aligned}
\Phi_{B} \leq & -k_{11 B} \frac{\left\|\tilde{\boldsymbol{r}}_{1}^{B \Gamma}\right\|^{2}}{\left(\Delta^{B \Gamma}\right)^{2}}-k_{12 B}\left\|\tilde{\boldsymbol{r}}_{2}^{B \Gamma}\right\|^{2}-k_{21 B}\left\|\operatorname{col}\left(q_{1}^{B \Gamma}-1, \overline{\boldsymbol{q}}^{B \Gamma}\right)\right\|^{2}-k_{22 B}\left\|\boldsymbol{\omega}^{B \Gamma}\right\|^{2}-k_{31 B}\left\|\boldsymbol{\vartheta}^{B \Gamma}\right\|^{2}-k_{31 B}^{N}\left(\left(\eta_{1}^{B \Gamma}\right)^{4}\right. \\
& \left.+\left(\eta_{2}^{B \Gamma}\right)^{4}+\left(\varepsilon^{B \Gamma}\right)^{4}\right)-k_{32 B}\left\|\boldsymbol{\mu}^{B \Gamma}\right\|^{2}-k_{32 B}^{N}\left(\left(\mu_{1}^{B \Gamma}\right)^{4}+\left(\mu_{2}^{B \Gamma}\right)^{4}+\left(\mu_{3}^{B \Gamma}\right)^{4}\right)+\gamma \Gamma(1-\varrho)\left(\frac{1}{4 \bar{\epsilon}_{31}}+\frac{1}{16 \bar{\epsilon}_{32}}+\frac{1}{12 \bar{\epsilon}_{32}^{4}}\right)
\end{aligned}
$$

where

$$
\begin{aligned}
k_{11 B} & =\lambda_{m}\left(\boldsymbol{K}_{1 B}\right) \gamma_{1}^{2}-\gamma \gamma_{1} \Gamma \epsilon_{21} k_{2 B}-\gamma_{1}\left(\epsilon_{31}+\frac{1}{2} \epsilon_{32}+\frac{1}{4} \epsilon_{32}^{4}\right)\left(G \bar{A}_{1} \vee G \bar{A}_{2} \vee E A\right), \\
k_{12 B} & =\lambda_{m}\left(\boldsymbol{K}_{1 B}\right)-\frac{\gamma \Gamma m_{0}}{2}-\gamma \epsilon_{22} k_{2 B}, \\
k_{21 B} & =\frac{1}{4} k_{2 B} \gamma_{2}^{2}-k_{2 B} \gamma \gamma_{2} \Gamma \epsilon_{23}-\gamma_{2}\left(\epsilon_{41}+\frac{1}{2} \epsilon_{42}+\frac{1}{4} \epsilon_{42}^{4}\right)\left(E I_{1} \vee E I_{2} \vee G I_{3}\right), \\
k_{22 B} & =k_{2 B}-\gamma \Gamma \lambda_{M}\left(\boldsymbol{J}_{0}\right)-k_{2 B} \gamma \Gamma \epsilon_{24}, \\
k_{31 B} & =\frac{5 \gamma \Gamma}{12}\left(G \bar{A}_{1} \wedge G \bar{A}_{2} \wedge E A\right)+\gamma^{2} \Gamma^{2} \lambda_{m}\left(\boldsymbol{K}_{1 B}\right)-\frac{1}{\epsilon_{21}} \gamma \gamma_{1} \Gamma k_{2 B}-\frac{1}{\epsilon_{22}} \gamma \Gamma k_{2 B} \\
& -\frac{\gamma_{1}}{4 \epsilon_{31}}\left(G \bar{A}_{1} \vee G \bar{A}_{2} \vee E A\right)-\gamma \Gamma(1-\varrho) \bar{\epsilon}_{31} E A, \\
k_{31 B}^{N} & =\frac{\gamma \Gamma}{24}\left(G \bar{A}_{1} \wedge G \bar{A}_{2} \wedge E A\right)-\gamma \Gamma\left(\frac{1}{8 \epsilon_{32}}+\frac{1}{12 \epsilon_{32}^{4 / 3}}\right)\left(G \bar{A}_{1} \vee G \bar{A}_{2} \vee E A\right)--\gamma \Gamma(1-\varrho)\left(\bar{\epsilon}_{32}+\frac{\bar{\epsilon}_{32}^{4 / 3}}{4}\right) E A, \\
k_{32 B} & =\frac{5 \gamma \Gamma}{12}\left(E I_{1} \wedge E I_{2} \wedge G I_{3}\right)+\gamma^{2} \Gamma^{2} k_{2 B}-\frac{1}{\epsilon_{23}} k_{2 B} \gamma \gamma_{2} \Gamma-\frac{1}{\epsilon_{24}} k_{2 B} \gamma \Gamma-\frac{\gamma_{1}}{4 \epsilon_{41}}\left(E I_{1} \vee E I_{2} \vee G I_{3}\right), \\
k_{32 B}^{N} & =\frac{\gamma \Gamma}{24}\left(E I_{1} \wedge E I_{2} \wedge G I_{3}\right)-\gamma \Gamma\left(\frac{1}{8 \epsilon_{42}}+\frac{1}{12 \epsilon_{42}^{4 / 3}}\right)\left(E I_{1} \vee E I_{2} \vee G I_{3}\right),
\end{aligned}
$$


with $\epsilon_{i j}, i=3,4 ; j=1,2, \bar{\epsilon}_{31}$, and $\bar{\epsilon}_{32}$ being positive constants to be determined. Now, Substituting (70) into (68) then together with (66) into (60) gives

$$
\begin{aligned}
\mathcal{L} U & \leq-k_{11}\|\boldsymbol{v}\|_{L^{2}}^{2}-k_{11}^{N}\left\langle 1, v_{1}^{4}+v_{2}^{4}+v_{3}^{4}\right\rangle_{L^{2}}-k_{12}\|\boldsymbol{\omega}\|_{L^{2}}^{2}-k_{12}^{N}\left\langle 1, \omega_{1}^{4}+\omega_{2}^{4}+\omega_{3}^{4}\right\rangle_{L^{2}} \\
& -k_{21}\|\boldsymbol{\vartheta}\|_{L^{2}}^{2}-k_{21}^{N}\left\langle 1, \eta_{1}^{4}+\eta_{2}^{4}+\varepsilon^{4}\right\rangle_{L^{2}}-k_{22}\|\boldsymbol{\mu}\|_{L^{2}}^{2}-k_{22}^{N}\left\langle 1, \mu_{1}^{4}+\mu_{2}^{4}+\mu^{4}\right\rangle_{L^{2}} \\
& -\left(\lambda_{m}\left(\boldsymbol{D}_{11}^{0}\right)-\epsilon_{310}\right)\left\|\tilde{\boldsymbol{r}}_{2}^{B 0}\right\|^{2}-\left(\lambda_{m}\left(\boldsymbol{D}_{21}^{0}\right)-\epsilon_{320}\right)\left\|\boldsymbol{\omega}^{B 0}\right\|^{2}-\epsilon_{11 B}\left\|\left(\gamma_{1} \frac{\tilde{r}_{1}^{B \Gamma}}{\Delta^{B \Gamma}}+\tilde{\boldsymbol{r}}_{2}^{B \Gamma}+\gamma \Gamma \boldsymbol{R}_{1}\left(\boldsymbol{q}^{B \Gamma}\right) \boldsymbol{\vartheta} \boldsymbol{\vartheta}^{B \Gamma}\right)\right\|^{2} \\
& -\epsilon_{21 B}\left\|\gamma_{2} \boldsymbol{K}^{T}\left(\boldsymbol{q}^{B \Gamma}\right) \operatorname{col}\left(q_{1}^{B \Gamma}-1, \overline{\boldsymbol{q}}^{B \Gamma}\right)+\boldsymbol{\omega}^{B \Gamma}+\gamma \Gamma \boldsymbol{\mu}^{B \Gamma}\right\|^{2}-k_{11 B} \frac{\left\|\tilde{\boldsymbol{r}}_{1}^{B \Gamma}\right\|^{2}}{\left(\Delta^{B \Gamma}\right)^{2}}-k_{12 B}\left\|\tilde{\boldsymbol{r}}_{2}^{B \Gamma}\right\|^{2} \\
& -k_{21 B}\left\|\operatorname{col}\left(q_{1}^{B \Gamma}-1, \overline{\boldsymbol{q}}^{B \Gamma}\right)\right\|^{2}-k_{22 B}\left\|\boldsymbol{\omega}^{B \Gamma}\right\|^{2}-k_{31 B}\left\|\boldsymbol{\vartheta}^{B \Gamma}\right\|^{2}-k_{31 B}^{N}\left(\eta_{1}^{4}+\eta_{2}^{4}+\varepsilon^{4}\right)-k_{32 B}\left\|\boldsymbol{\mu}^{B \Gamma}\right\|^{2} \\
& -k_{32 B}^{N}\left(\mu_{1}^{4}+\mu_{2}^{4}+\mu_{3}^{4}\right)+c_{0},
\end{aligned}
$$

where

$$
\begin{aligned}
c_{0} & =\left(\frac{1}{4 \epsilon_{110}}+\frac{\gamma \Gamma}{4 \epsilon_{140}}+\frac{\gamma(1-\varrho) \Gamma}{4 \bar{\epsilon}_{130}}\right)\left\|\boldsymbol{f}_{10}\right\|_{L^{2}}^{2}+\left(\frac{1}{4 \epsilon_{210}}+\frac{\gamma \Gamma}{4 \epsilon_{240}}\right)\left\|\boldsymbol{f}_{20}\right\|_{L^{2}}^{2}+\frac{1}{4 \epsilon_{310}}\left\|\boldsymbol{f}_{10}^{B 0}\right\|^{2}+\frac{1}{4 \epsilon_{320}}\left\|\boldsymbol{f}_{20}^{B 0}\right\|^{2} \\
& +\frac{1}{4 \epsilon_{12 B}}\left\|\boldsymbol{f}_{10}^{B \Gamma}\right\|^{2}+\frac{1}{4 \epsilon_{22}}\left\|\boldsymbol{f}_{20}^{B \Gamma}\right\|^{2}+(1-\varrho) E A \Gamma\left(\frac{1}{4 \bar{\epsilon}_{12}}+\frac{1}{16 \bar{\epsilon}_{13}}+\frac{1}{12 \bar{\epsilon}_{13}^{4}}\right) \\
& +\gamma \Gamma(1-\varrho)\left(\frac{\lambda_{M}\left(\boldsymbol{D}_{11}\right)}{4 \bar{\epsilon}_{110}}+\frac{\lambda_{M}\left(\boldsymbol{D}_{12}\right)}{4 \bar{\epsilon}_{120}^{4}}+\bar{\epsilon}_{130} \Gamma\right)+\gamma \Gamma(1-\varrho)\left(\frac{1}{4 \bar{\epsilon}_{31}}+\frac{1}{16 \bar{\epsilon}_{32}}+\frac{1}{12 \bar{\epsilon}_{32}^{4}}\right) .
\end{aligned}
$$

We now choose the constants $\varrho, \gamma, \gamma_{1}, \gamma_{2}, \boldsymbol{K}_{1 B}, k_{2 B}, \epsilon_{i j B},(i, j)=1,2 ; \epsilon_{i j 0}, i=1,2, j=1, \ldots, 4$; $\epsilon_{2 j}, j=1, \ldots, 4 ; \epsilon_{i j}, i=3,4, j=1,2 ; \bar{\epsilon}_{3 i}, i=1,2 ; \bar{\epsilon}_{1 i}, i=2,3$; and $\bar{\epsilon}_{1 i 0}, i=1,2,3$ such that

$$
\begin{aligned}
& k_{11} \geq c_{0} \lambda_{M}\left(\boldsymbol{K}_{1 B}\right), k_{i j} \geq k_{i j}^{\diamond}, k_{i j}^{N} \geq k_{i j}^{N \diamond},(i, j)=1,2 \\
& k_{i j B} \geq k_{i j B}^{\diamond}, k_{i j B}^{N} \geq k_{i j B}^{N \diamond}, i=1,2,3, j=1,2, \\
& \left(\lambda_{m}\left(\boldsymbol{D}_{11}^{0}\right)-\epsilon_{310}\right) \geq k_{110}^{\diamond},\left(\lambda_{m}\left(\boldsymbol{D}_{21}^{0}\right)-\epsilon_{320}\right) \geq k_{210}^{\diamond},
\end{aligned}
$$

where $k_{i j}^{\diamond}, k_{i j}^{N \diamond}$ with $(i, j)=1,2, k_{i j B}^{\diamond}, k_{i j B}^{N \diamond}$ with $i=1,2,3, j=1,2, k_{110}^{\diamond}$, and $k_{210}^{\diamond}$ are positive constants. We elaborate the choice of $\varrho_{0 i}, i=1,2 ; \varrho, \gamma, \gamma_{1}, \gamma_{2}, \boldsymbol{K}_{1 B}, k_{2 B}, \epsilon_{i j B},(i, j)=1,2 ; \epsilon_{i j 0}, i=1,2, j=1, \ldots, 4$; $\epsilon_{2 j}, j=1, \ldots, 4 ; \epsilon_{i j}, i=3,4, j=1,2 ; \bar{\epsilon}_{3 i}, i=1,2 ; \bar{\epsilon}_{1 i}, i=2,3$; and $\bar{\epsilon}_{1 i 0}, i=1,2,3$ to ensure that the conditions listed in (44) and (74) hold in the following remark.

\section{Remark 5.3.}

1) The constant $c_{1}$ : From (43), it can be seen that by choosing a small $\gamma$ the inequality (44) holds for any given positive constants $\varrho_{01}, \varrho_{02}$, and $0 \leq \varrho \leq 1$.

2) The constants $\left(k_{i j}, k_{i j}^{N}\right),(i, j)=1,2$ : If the bending stiffness is strictly larger than the shear stiffness multiplied by square of the beam's length, i.e.,

$$
\left(E I_{1} \wedge E I_{2}\right) \gg \Gamma^{2}\left(G \bar{A}_{1} \vee G \bar{A}_{2}\right)
$$

then the constants $\left(k_{i j}, k_{i j}^{N}\right),(i, j)=1,2$ can be made strictly positive by choosing $\varrho=1 ; \Gamma \epsilon_{12}<\frac{1}{6}$; $\Gamma\left(\epsilon_{13}^{2}+\frac{1}{4} \epsilon_{13}^{4 / 3}\right)<\frac{1}{6} ;$ small $\left(\gamma, \epsilon_{110}, \epsilon_{210}, \epsilon_{140}, \epsilon_{240}, \epsilon_{14}\right)$; and large $\left(\epsilon_{120}, \epsilon_{130}, \epsilon_{220}, \epsilon_{230}\right)$. In this case, under no external disturbances, the closed-loop system is globally asymptotically stable at the origin as seen by substituting $f_{i 0}=0, f_{i 0}^{B 0}=0, f_{i 0}^{B \Gamma}=0$, and $\varrho=1$ into (43) and (73). This is because the beam is easier to be sheared than to be bent. However, if the condition (75) does not hold, the constant $\varrho$ needs to be a small positive constant. In this case, the closed loop system is globally practically asymptotically stable at the origin even under no external disturbances.

3) The constants $\left(k_{i j B}, k_{i j B}^{N}\right), i=1,2,3, j=1,2$, ): Interpretation of how to ensure these constants strictly positive can be carried out similarly to the constants $\left(k_{i j}, k_{i j}^{N}\right),(i, j)=1,2$.

This remark suggests the following procedure to choose $\varrho_{0 i}, i=1,2 ; \varrho, \gamma, \gamma_{1}, \gamma_{2}, \boldsymbol{K}_{1 B}, k_{2 B}, \epsilon_{i j B},(i, j)=$ 1,$2 ; \epsilon_{i j 0}, i=1,2, j=1, \ldots, 4 ; \epsilon_{2 j}, j=1, \ldots, 4 ; \epsilon_{i j}, i=3,4, j=1,2 ; \bar{\epsilon}_{3 i}, i=1,2 ; \bar{\epsilon}_{1 i}, i=2,3$; and $\bar{\epsilon}_{1 i 0}, i=1,2,3$ to ensure that the conditions listed in (44) and (74) hold. 


\section{Procedure 5.1.}

Step 1. Pick positive constants $\varrho_{01}, \varrho_{02}$, and $0 \leq \varrho \leq 1$. Then from (43), find a range of $\gamma$ such that the inequality (44) holds. This gives the first range of $\gamma$.

Step 2. Check the condition (75). If this condition holds, pick $\varrho=1 ; \operatorname{small}\left(\gamma, \epsilon_{110}, \epsilon_{210}, \epsilon_{140}, \epsilon_{240}, \epsilon_{14}\right)$; and large $\left(\epsilon_{120}, \epsilon_{130}, \epsilon_{220}, \epsilon_{230}\right)$ so that $\left(k_{i j}, k_{i j}^{N}\right),(i, j)=1,2$ are strictly positive. If the condition (75) does not hold, pick $0 \leq \varrho<1$. Then carry out as above. This step gives the second range of $\gamma$.

Step 3. Pick small $\left(\gamma, \gamma_{1}, \gamma_{2}, \epsilon_{2 j}, j=1, \ldots, 4, \epsilon_{i j}, i=3,4 ; j=1,2, \bar{\epsilon}_{31}, \bar{\epsilon}_{32}, \epsilon_{i j B},(i, j)=1,2\right)$ and large $\left(\boldsymbol{K}_{1 B}, k_{2 B}\right)$ such that $\left.\left(k_{i j B}, k_{i j B}^{N}\right), i=1,2,3, j=1,2,\right)$ are strictly positive. This step gives the third range of $\gamma$. The desired range of $\gamma$ is the smallest range of those in Steps 1, 2, and 3.

With (74), we can write (72) as

$$
\mathcal{L} U \leq-c_{3} \mathcal{E}+c_{0}
$$

where

$$
\begin{aligned}
c_{3}= & k_{11} \wedge k_{11}^{N} \wedge k_{12} \wedge k_{12}^{N} \wedge k_{21} \wedge k_{22}^{N} \wedge k_{21} \wedge k_{22}^{N} \wedge k_{110}^{\diamond} \wedge k_{210}^{\diamond} \wedge \epsilon_{11 B} \wedge \epsilon_{21 B} \wedge \frac{2 k_{11 B}}{\lambda_{M}\left(\boldsymbol{K}_{1 B}\right)\left(\Delta_{M}^{B \Gamma}\right)^{2}} \\
& \wedge k_{12 B} \wedge k_{21 B} \wedge k_{22 B} \wedge k_{31 B}^{N} \wedge k_{32 B}^{N}, \wedge\left(\lambda_{m}\left(\boldsymbol{D}_{11}^{0}\right)-\epsilon_{310}\right) \wedge\left(\lambda_{m}\left(\boldsymbol{D}_{21}^{0}\right)-\epsilon_{320}\right),
\end{aligned}
$$

with $\Delta_{M}^{B \Gamma}$, which depends on the initial values, being the maximum value of $\Delta^{B \Gamma}$. It is noted that in order to obtain (77) we have used (74), particularly the first inequality in (74), to ensure that $U$ is bounded by the initial values and then used the inequality $\sqrt{1+\boldsymbol{x}^{T} \boldsymbol{K}_{1 B} \boldsymbol{x}}-1 \leq \frac{\lambda_{M}\left(\boldsymbol{K}_{1 B}\right)}{2}\|\boldsymbol{x}\|^{2}$ for all $\boldsymbol{x} \in \mathbb{R}^{3}$. We summarize the main results in the following theorem.

Theorem 5.1. Under Assumption 3.1, the boundary control vectors $\phi_{i B}, i=1,2$ given in (63) solves Control Objective 3.1 provided that $\varrho_{0 i}, i=1,2 ; \varrho, \gamma, \gamma_{1}, \gamma_{2}, K_{1 B}, k_{2 B}, \epsilon_{i j B},(i, j)=1,2 ; \epsilon_{i j 0}, i=$ $1,2, j=1, \ldots, 4 ; \epsilon_{2 j}, j=1, \ldots, 4 ; \epsilon_{i j}, i=3,4, j=1,2 ; \bar{\epsilon}_{3 i}, i=1,2 ; \bar{\epsilon}_{1 i}, i=2,3$; and $\bar{\epsilon}_{1 i 0}, i=1,2,3$ are chosen such that the conditions in (44) and (74) hold. The closed-loop system consisting of (34), (36), and (63) is globally well-posed and globally practically asymptotically stable at the origin.

Proof. We first define

$$
\boldsymbol{X}=\left[\begin{array}{c}
\tilde{\boldsymbol{r}}_{1} \\
\boldsymbol{q} \\
\boldsymbol{v} \\
\boldsymbol{\omega} \\
\tilde{\boldsymbol{r}}_{1}^{B 0} \\
\boldsymbol{q}^{B 0} \\
\tilde{\boldsymbol{r}}_{2}^{B 0} \\
\boldsymbol{\omega}^{B 0} \\
\tilde{\boldsymbol{r}}_{1}^{B \Gamma} \\
\boldsymbol{q}^{B \Gamma} \\
\tilde{\boldsymbol{r}}_{2}^{B \Gamma} \\
\boldsymbol{\omega}^{B \Gamma}
\end{array}\right], \quad \boldsymbol{F}(\boldsymbol{X}, t)=\left[\begin{array}{l}
\boldsymbol{R}_{1}(\boldsymbol{q}) \boldsymbol{v} \\
\boldsymbol{K}(\boldsymbol{q}) \boldsymbol{\omega} \\
\frac{1}{m_{0}}\left(m_{0}\left(\boldsymbol{R}_{1}^{-1}(\boldsymbol{q})\right)_{t} \boldsymbol{R}_{1}(\boldsymbol{q}) \boldsymbol{v}+\boldsymbol{R}_{1}^{-1}(\boldsymbol{q}) \mathbb{D} \boldsymbol{n}+\boldsymbol{f}_{1}\right) \\
\boldsymbol{J}_{0}^{-1}\left(\mathbb{D} \boldsymbol{m}-\boldsymbol{\omega} \times\left(\boldsymbol{J}_{0} \boldsymbol{\omega}\right)+\mathbb{D} \boldsymbol{r} \times \boldsymbol{n}+\boldsymbol{f}_{2}\right) \\
\tilde{\boldsymbol{r}}_{2}^{B 0} \\
\boldsymbol{K}\left(\boldsymbol{q}^{B 0}\right) \boldsymbol{\omega}^{B 0} \\
\frac{1}{m_{P}}\left(\boldsymbol{n}^{B 0}+\boldsymbol{f}_{1}^{B 0}\right) \\
\boldsymbol{J}_{P}^{-1}\left(\boldsymbol{m}^{B 0}+\boldsymbol{f}_{2}^{B 0}\right) \\
\tilde{\boldsymbol{r}}_{2}^{B \Gamma} \\
\boldsymbol{K}\left(\boldsymbol{q}^{B \Gamma}\right) \boldsymbol{\omega}^{B \Gamma} \\
\boldsymbol{M}_{H}^{-1}\left(-\boldsymbol{n}^{B \Gamma}+\boldsymbol{\phi}_{1 B}+\boldsymbol{f}_{1}^{B \Gamma}\right) \\
\boldsymbol{J}_{H}^{-1}\left(-\boldsymbol{m}^{B \Gamma}+\boldsymbol{\phi}_{2 B}+\boldsymbol{f}_{2}^{B \Gamma}\right)
\end{array}\right]
$$

where $\boldsymbol{\phi}_{i B}, i=1,2$ are defined in Eq. (63). Next, we can write Eqs. (34) and (36) as follows

$$
\frac{d \boldsymbol{X}}{d t}=\boldsymbol{F}(\boldsymbol{X}, t)
$$

For well-posedness and stability analysis of Eq. (79), we introduce the functional spaces: $H=$ $\left(W^{1,2}(\mathcal{D})\right)^{6} \times\left(L^{2}(\mathcal{D})\right)^{6} \times \mathbb{R}^{12}, V=\left(W^{2,2}(\mathcal{D})\right)^{6} \times\left(L^{2}(\mathcal{D})\right)^{6} \times \mathbb{R}^{12}, V^{*}=\left(W^{-2,2}(\mathcal{D})\right)^{6} \times\left(L^{2}(\mathcal{D})\right)^{6} \times \mathbb{R}^{12}$, where $\mathcal{D}:=(0, \Gamma)$, and $W^{-m, p}(\mathcal{D})$ denotes the dual of $W^{m, p}(\mathcal{D})$. Then, we have the embedding $V \subset H \equiv H^{*} \subset V^{*}$. Let

$$
\hat{\boldsymbol{X}}=\operatorname{col}\left(\hat{\boldsymbol{r}}_{1}, \hat{\boldsymbol{q}}, \hat{\boldsymbol{v}}, \hat{\boldsymbol{\omega}}, \hat{\boldsymbol{r}}_{1}^{B 0}, \hat{\boldsymbol{q}}^{B 0}, \hat{\boldsymbol{\boldsymbol { r }}}_{2}^{B 0}, \hat{\boldsymbol{\omega}}^{B 0}, \hat{\boldsymbol{r}}_{1}^{B \Gamma}, \hat{\boldsymbol{q}}^{B \Gamma}, \hat{\boldsymbol{r}}_{2}^{B \Gamma}, \hat{\boldsymbol{\omega}}^{B \Gamma}\right) .
$$


Define

$$
\begin{aligned}
\langle\boldsymbol{X}, \hat{\boldsymbol{X}}\rangle_{H} & =\frac{m_{0}}{2}\langle\boldsymbol{v}, \hat{\boldsymbol{v}}\rangle_{L^{2}}+\frac{1}{2}\left\langle\boldsymbol{\omega}, \boldsymbol{J}_{0} \hat{\boldsymbol{\omega}}\right\rangle_{L^{2}}+\sum_{i=1}^{2} G \bar{A}_{i}\left\langle\eta_{i},\left(\frac{1}{2} \hat{\eta}_{i}-\frac{1}{6} \hat{\eta}_{i}^{2}+\frac{1}{12} \hat{\eta}_{i}^{3}\right)\right\rangle_{L^{2}}+E A\left\langle\varepsilon,\left(\frac{1}{2} \hat{\boldsymbol{\varepsilon}}-\frac{1}{6} \hat{\boldsymbol{\varepsilon}}^{2}+\frac{1}{12} \hat{\varepsilon}^{3}\right)\right\rangle_{L^{2}} \\
& +\sum_{i=1}^{2} E I_{i}\left\langle\mu_{i},\left(\frac{1}{2} \hat{\mu}_{i}-\frac{1}{6} \hat{\mu}_{i}^{2}+\frac{1}{12} \hat{\mu}_{i}^{3}\right)\right\rangle_{L^{2}}+G I_{3}\left\langle\mu_{3},\left(\frac{1}{2} \hat{\mu}_{3}-\frac{1}{6} \hat{\mu}_{3}^{2}+\frac{1}{12} \hat{\mu}_{3}^{3}\right)\right\rangle_{L^{2}}+\gamma m_{0}\langle\boldsymbol{\vartheta}, \hat{\boldsymbol{v}} s\rangle_{L^{2}} \\
& +\gamma\left\langle\boldsymbol{\mu}, \boldsymbol{J}_{0} \hat{\boldsymbol{\omega}} s\right\rangle_{L^{2}}+\frac{m_{P}}{2}\left\langle\tilde{\boldsymbol{r}}_{2}^{B 0}, \hat{\tilde{\boldsymbol{r}}}_{2}^{B 0}\right\rangle_{L^{2}}+\frac{1}{2}\left(\boldsymbol{\omega}^{B 0}\right)^{T} \boldsymbol{J}_{P} \hat{\boldsymbol{\omega}}^{B 0}+\frac{1}{2}\left(\gamma_{1} \frac{\tilde{\boldsymbol{r}}_{1}^{B \Gamma}}{\Delta^{B \Gamma}}+\tilde{\boldsymbol{r}}_{2}^{B \Gamma}+\gamma \Gamma \boldsymbol{R}_{1}\left(\boldsymbol{q}^{B \Gamma}\right) \boldsymbol{\vartheta}^{B \Gamma}\right)^{T} \boldsymbol{M}_{H} \\
& \times\left(\gamma_{1} \hat{\hat{\boldsymbol{r}}}_{1}^{B \Gamma}+\tilde{\boldsymbol{r}}_{2}^{B \Gamma}+\gamma \Gamma \boldsymbol{R}_{1}\left(\hat{\boldsymbol{q}}^{B \Gamma}\right) \hat{\boldsymbol{\vartheta}}^{B \Gamma}\right)+\frac{1}{2}\left(\gamma_{2} \boldsymbol{K}^{T}\left(\boldsymbol{q}^{B \Gamma}\right) \operatorname{col}\left(q_{1}^{B \Gamma}-1, \overline{\boldsymbol{q}}^{B \Gamma}\right)+\boldsymbol{\omega}^{B \Gamma}+\gamma \Gamma \boldsymbol{\mu}^{B \Gamma}\right)^{T} \boldsymbol{J}_{H} \\
& \times\left(\gamma_{2} \boldsymbol{K}^{T}\left(\hat{\boldsymbol{q}}^{B \Gamma}\right) \operatorname{col}\left(\hat{\boldsymbol{q}}_{1}^{B \Gamma}-1, \hat{\overline{\boldsymbol{q}}}^{B \Gamma}\right)+\hat{\boldsymbol{\omega}}^{B \Gamma}+\gamma \Gamma \hat{\boldsymbol{\mu}}^{B \Gamma}\right)+\gamma_{1}\left(\Delta^{B \Gamma}-1\right)+\gamma_{1}\left(\hat{\Delta}^{B \Gamma}-1\right) \\
& +\frac{1}{2} \gamma_{2} k_{2 B}\left\|\operatorname{col}\left(q_{1}^{B \Gamma}-1, \overline{\boldsymbol{q}}^{B \Gamma}\right)\right\|^{2}+\frac{1}{2} \gamma_{2} k_{2 B}\left\|\operatorname{col}\left(\hat{q}_{1}^{B \Gamma}-1, \hat{\overline{\boldsymbol{q}}}^{B \Gamma}\right)\right\|^{2},
\end{aligned}
$$

where $\hat{\eta}_{i}, i=1,2, \hat{\mu}_{i}, i=1,2,3, \hat{\varepsilon}$, and $\hat{\boldsymbol{\vartheta}}^{B \Gamma}$ are the values of $\eta_{i}, i=1,2, \mu_{i}, i=1,2,3$, $\varepsilon$, and $\boldsymbol{\vartheta}^{B \Gamma}$ with $\mathbb{D} \tilde{\boldsymbol{r}}_{1}$ and $\boldsymbol{q}$ being replaced by $\mathbb{D} \hat{\tilde{\boldsymbol{r}}}_{1}$ and $\hat{\boldsymbol{q}}$, respectively. The constants $\gamma, \gamma_{1}, \gamma_{2}, k_{2 B}$, and the matrix $\boldsymbol{K}_{2 B}$ the conditions specified in Theorem 5.1. Let us denote by $\langle\boldsymbol{X}, \hat{\boldsymbol{X}}\rangle_{L H}$ linearization of $\langle\boldsymbol{X}, \hat{\boldsymbol{X}}\rangle_{H}$ at the origin. Then, it can be verified that $\langle\boldsymbol{X}, \hat{\boldsymbol{X}}\rangle_{L H}$ is a inner product with the norm $\langle\boldsymbol{X}, \boldsymbol{X}\rangle_{L H}=\|\boldsymbol{X}\|_{L H}^{2}$. In fact, there exist strictly positive constants $\bar{c}_{01}$ and $\bar{c}_{02}$ such that $\bar{c}_{01} \mathcal{E}_{L H} \leq\|X\|_{L H}^{2} \leq \bar{c}_{02} \mathcal{E}_{L H}$ locally, where $\mathcal{E}_{L H}$ is the linearization of $\mathcal{E}$, which is defined in Eq. (19).

We now verify all the conditions of Theorem 4.1. The continuity condition in Assumption 4.1 holds due to continuity of $\boldsymbol{F}(\boldsymbol{X}, t)$. By using $\langle\boldsymbol{X}-\hat{\boldsymbol{X}}, \boldsymbol{F}(\boldsymbol{X}, t)-\boldsymbol{F}(\hat{\boldsymbol{X}}, t)\rangle_{V V^{*}}=\langle\boldsymbol{X}-\hat{\boldsymbol{X}}, \boldsymbol{F}(\boldsymbol{X}, t)-\boldsymbol{F}(\hat{\boldsymbol{X}}, t)\rangle_{H}$ with the use of the local inner product in $L H$ defined as above and integration by parts similarly to the calculation of $\mathcal{L} U$ in Section 5.2, it is readily shown that the local monotonicity condition (28) and local growth condition (29) hold. From Eqs. (42) and (76) with $\mathcal{E}$ defined in Eq. (19), the conditions (30) and (33) hold with $\varrho_{1}=0, \varrho_{2}=0, \alpha_{1}\left(\|\boldsymbol{X}\|_{H}^{2}\right) \equiv c_{1}\|\boldsymbol{X}\|_{H}^{2}$ and $\alpha_{2}\left(\|\boldsymbol{X}\|_{H}^{2}\right) \equiv c_{2}\|\boldsymbol{X}\|_{H}^{2}$, where $c_{1}$ and $c_{2}$ are defined in Eq. (43). Proof of Theorem 5.1 is therefore completed.

\section{Simulation results}

This section illustrates the effectiveness of the proposed boundary controller on a steel beam with a cross section of an I-shape as shown in Fig. 2 and a length of $\Gamma=10 \mathrm{~m}$. Referring to Fig. 2, we have $A=10^{-4} \times 250 \mathrm{~m}^{2}, A_{1}=10^{-6} \times 8335 \mathrm{~m}^{2}, A_{2}=10^{-2} \times 1.835 \mathrm{~m}^{2}$, $I_{1}=10^{-8} \times 12083 \mathrm{~m}^{4}, I_{2}=10^{-8} \times 6770 \mathrm{~m}^{4}$, and $I_{3}=10^{-8} \times 2083 \mathrm{~m}^{4}$. The other parameters are $E=2.04 \times 10^{10} \mathrm{~kg} / \mathrm{m}^{2}$ and $G=8.4 \times 10^{10} \mathrm{~kg} / \mathrm{m}^{2}$. The payload is a solid steel sphere with a radius of $1 \mathrm{~m}$. Thus, $m_{P}=3.29 \times 10^{4} \mathrm{~kg}$ and $\boldsymbol{J}_{P}=1.32 \times 10^{4} \boldsymbol{I}_{3} \mathrm{~kg} \mathrm{~m}^{2}$. We take $\boldsymbol{M}_{H}=$ $3 m_{P} \operatorname{diag}(1,2.5,4.5) ; \boldsymbol{J}_{H}=\boldsymbol{J}_{P} \operatorname{diag}(1,2.5,4.5) ; \boldsymbol{D}_{11}^{0}=3 \times 10^{3} \boldsymbol{I}_{3} \mathrm{~kg} / \mathrm{s}$; $\boldsymbol{D}_{11}^{\Gamma}=10 \boldsymbol{D}_{11}^{0} ; \boldsymbol{D}_{21}^{0}=1.3 \times 10^{3} \mathrm{kgm}^{2} / \mathrm{s} ; \boldsymbol{D}_{21}^{\Gamma}=10 \times \boldsymbol{D}_{12}^{9} ;$ and the nonlinear damping matrices equal to $50 \%$ of the linear ones. The external

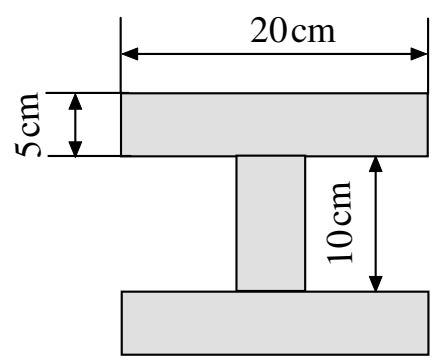

Figure 2: Dimensions of the beam's cross section. loads $\boldsymbol{f}_{10}:=\operatorname{col}\left(f_{110}, f_{120}, f_{130}\right)$ and $\boldsymbol{f}_{20}:=\operatorname{col}\left(f_{210}, f_{220}, f_{230}\right)$ are taken as

$$
f_{k i 0}=C_{D k i} \frac{\varrho_{w} d_{o}}{2} \sqrt{\frac{8}{\pi}} \sigma_{k i}(t)
$$

for $k=1,2$ and $i=1,2,3$, where $\varrho_{w}=1$ is the air density; $C_{D k i}=1.2$ are the drag coefficients; and $\sigma_{k i}(t)$ is the root mean square of the air particle velocity. The air particle velocities $\vartheta_{i}$ are expressed as:

$$
\vartheta_{i}(t)=\sum_{j=1}^{N_{i}} A_{i j} \omega_{i j} \sin \left(\omega_{i j} t+2 \pi \xi_{i j}\right)
$$

where $\xi_{i j}$ is a random number between 0 and 1 ; the amplitude $A_{i j}$, and frequency $\omega_{i j}$ of the $j^{\text {th }}$ wave are

$$
\omega_{i j}=\omega_{i m}-\frac{\omega_{i M}-\omega_{i m}}{N_{i}} j, S_{i j}=\frac{1.25}{4} \frac{\omega_{i o}^{4}}{\omega_{i j}^{4}} H_{i}^{2} e^{-1.25 \frac{\omega_{i o}^{4}}{\omega_{i j}^{4}}}, A_{i j}=\sqrt{2 S_{i j} \frac{\omega_{i M}-\omega_{i m}}{N_{i}}} .
$$



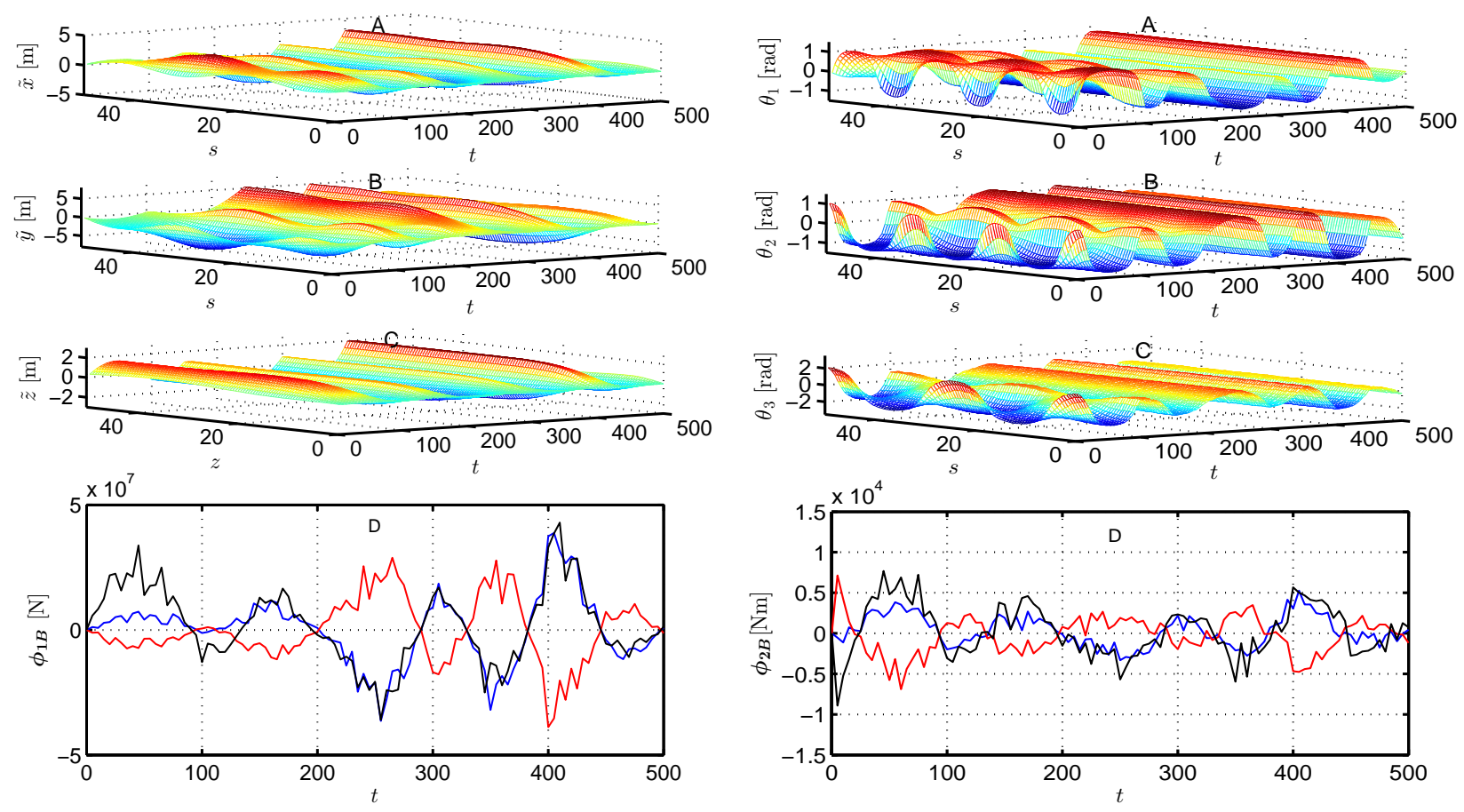

(a) Translational displacements and boundary control(b) Rotational angles and boundary control $\phi_{2 B}$, $\left(\phi_{21 B}\right.$ : $\phi_{1 B}\left(\phi_{11 B}\right.$ : black, $\phi_{12 B}$ : blue, $\phi_{13 B}$ : red).

black, $\phi_{22 B}$ : blue, $\phi_{23 B}$ : red).

Figure 3: Simulation results with gradient feedbacks

In (84), the minimum and maximum wave frequencies are $\omega_{i m}=0.2 \frac{\mathrm{rad}}{\mathrm{s}}, \omega_{i M}=5 \frac{\mathrm{rad}}{\mathrm{s}}$; the two-parameter Bretschneider spectrum $S_{i j}$ are used with the significant wave heights $H_{1}=H_{2}=2 \mathrm{~m}$ and $H_{3}=0.5 \mathrm{~m}$; the modal frequency is $\omega_{i o}=\frac{2 \pi}{T_{i}}$ with the period $T_{1}=T_{2}=7.8, T_{3}=2.5 ; N_{i}=10$. The external loads at boundaries are taken as $\boldsymbol{f}_{i 0}^{B 0}=5 \boldsymbol{f}_{i 0}$ and $\boldsymbol{f}_{i 0}^{B \Gamma}=10 \boldsymbol{f}_{i 0}$.

The control gains are chosen as follows: $\varrho=0.1, \gamma=\frac{1}{100 \Gamma}, \gamma_{1}=\gamma_{2}=3 \gamma \Gamma, \boldsymbol{K}_{1 B}=5 \boldsymbol{I}_{3} E A$, $k_{2 B}=5 E A$, and $\epsilon_{i j B}=\frac{1}{2} E A,(i j)=1,2$. It is readily checked that the above choice of the control gains ensures that all the conditions specified in Theorem 5.1 hold for some positive constants $\varrho_{0 i}, i=1,2$; $\epsilon_{i j 0}, i=1,2, j=1, \ldots, 4 ; \epsilon_{2 j}, j=1, \ldots, 4 ; \epsilon_{i j}, i=3,4, j=1,2 ; \bar{\epsilon}_{3 i}, i=1,2 ; \bar{\epsilon}_{1 i}, i=2,3$; and $\bar{\epsilon}_{1 i 0}, i=$ $1,2,3$. The initial conditions are taken as $t_{0}=0 ; \tilde{\boldsymbol{r}}_{10}(s)=\operatorname{col}\left(1.5 \sin \left(\frac{4 \pi s}{\Gamma}\right), 2 \cos \left(\frac{4 \pi s}{\Gamma}\right),-0.8 \sin \left(\frac{2 \pi s}{\Gamma}\right)\right)$; $\boldsymbol{\theta}_{0}(s)=\operatorname{col}\left(0.5 \sin \left(\frac{4 \pi s}{\Gamma}\right), 0.5 \cos \left(\frac{4 \pi s}{\Gamma}\right), \sin \left(\frac{2 \pi s}{\Gamma}\right)\right)$; and all other initial values are chosen to be zero.

We run two simulations. In the first simulation, only gradients are used for feedback, i.e.,

$$
\begin{aligned}
& \boldsymbol{\phi}_{1 B}=-\left(\boldsymbol{K}_{1 B}+\boldsymbol{I}_{3}\left(\epsilon_{11 B}+\epsilon_{12 B}\right)\right) \gamma \Gamma \boldsymbol{R}_{1}\left(\boldsymbol{q}^{B \Gamma}\right) \boldsymbol{\vartheta}^{B \Gamma}, \\
& \boldsymbol{\phi}_{2 B}=-\left(k_{2 B}+\epsilon_{21 B}+\epsilon_{22 B}\right) \gamma \Gamma \boldsymbol{\mu}^{B \Gamma} .
\end{aligned}
$$

This is similar to the case where the actuated end of the beam is clamped. In the second simulation, the proposed boundary controls (63) is used. In both simulations, the length of simulation time is 500 seconds. Moreover, the second order (in space and time) centered, explicit finite difference scheme is used to numerically solve the partial differential equations (4) together with the boundary conditions (15) where the boundary controls $\phi_{i B}, i=1,2,3$ given in (63). The time step is $\Delta t=0.01$ and space step is $\Delta z=0.2$. This choice ensures that the convergence parameter $r=\frac{\Delta t}{(\Delta z)^{2}}=0.25$ is positive and less than 0.5 as required for stable solutions [42].

Case with only gradient feedbacks (85): The results are plotted in Fig. $3 \mathrm{a}$ and Fig. $3 \mathrm{~b}$. The displacements $(\tilde{x}(s, t), \tilde{y}(s, t), \tilde{z}(s, t))$ are plotted in Sub-figs. 3a.A, 3a.B and 3a.C, the rotations $\left(\theta_{1}(s, t), \theta_{2}(s, t), \theta_{3}(s, t)\right)$ are plotted in Sub-figs. 3b.A, 3b.B and 3b.C, while the controls $\boldsymbol{\phi}_{1 B}$ and $\boldsymbol{\phi}_{2 B}$ are plotted in Sub-fig. 3a.D and Sub-fig. 3b.D. It is seen that the displacements and rotations oscillate with quite large magnitudes due to the external loads but are bounded due to the gradient feedbacks, 

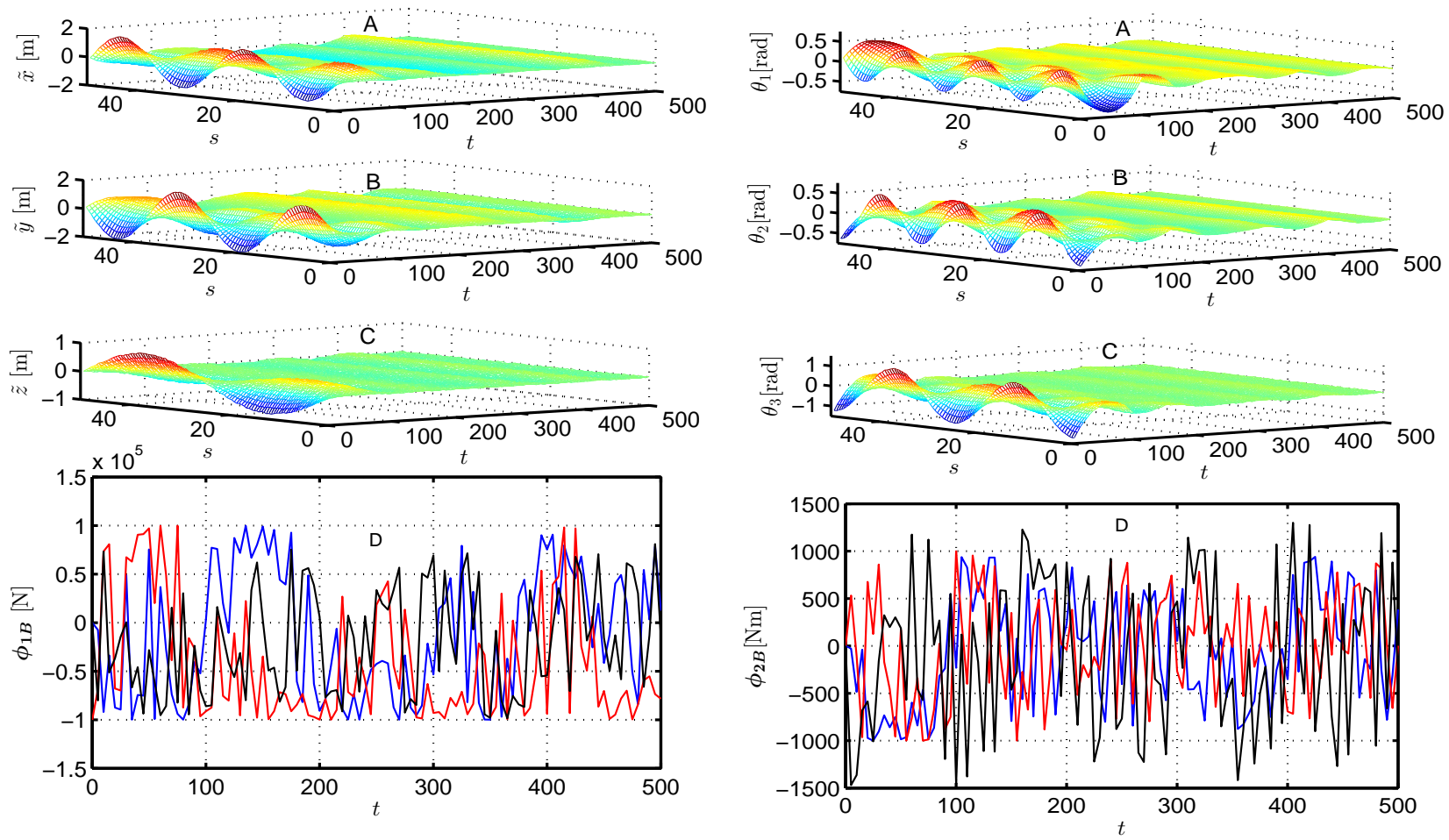

(a) Translational displacements and boundary control(b) Rotational angles and boundary control $\phi_{2 B},\left(\phi_{21 B}\right.$ : $\phi_{1 B}\left(\phi_{11 B}\right.$ : black, $\phi_{12 B}$ : blue, $\phi_{13 B}$ : red).

black, $\phi_{22 B}$ : blue, $\phi_{23 B}$ : red).

Figure 4: Simulation results with the proposed boundary controls

structural stiffness, and damping. Note that the magnitude of $\tilde{z}(s, t)$ is much smaller than $\tilde{x}(s, t)$ and $\tilde{y}(s, t)$ because the beam is easier to be bent/sheared than to be extended. For quantitative comparison, the total deformation $E_{T}=\int_{0}^{\Gamma}\left[\|\boldsymbol{v}(s, t)\|^{2}+\|\omega(s, t)\|^{2}+\|\boldsymbol{\vartheta}(s, t)\|^{2}+\|\boldsymbol{\mu}(s, t)\|^{2}\right] d s$ is also numerically calculated. This gives $E_{T}=1911.8$.

Case with the proposed feedbacks (63): The results are plotted in Fig.4a and Fig. 4b. Comparing Sub-figs. (4a.A, 4a.B, 4a.C, 4b.A, 4b.B, 4b.C) in this case with corresponding Sub-figs. (3a.A, 4a.B, 3a.C, 3b.A, 3b.B, 3b.C) in the case where only gradient feedbacks are applied clearly shows that a significant reduction (about 15 times less) in magnitude of all displacements and rotations. Finally, it is noted that the controls $\phi_{1 B}$ and $\phi_{2 B}$ are quite large for the case with only gradient feedbacks in comparison with those for the proposed controls as seen from Sub-figs (3a.D, (3b.D) and Sub-figs. (4a.D, (4b.D) due to larger translational and rotational motions for the case only gradient feedbacks than those for the case with the proposed controls. This control gives $E_{T}=142.3$

\section{Conclusions}

The exact nonlinear model describing nonlinear couple motions of Timoshenko beams under external loads in space was derived and used for boundary control design. The developed boundary control laws stabilize both large and small amplitudes of translational and rotational motions of the beams and their gradients, and fully consider the coupling between all the motions. These control laws ensure that the closed-loop system is globally practically asymptotically stable at the origin. Analysis of both well-posedness and stability was carried out based on a Lyapunov-type theorem, which was developed to study well-posedness and stability for a class of evolution systems. Future work is to consider spinning Timoshenko beams such as spindles and drillers in space. This will require a further development of the control design proposed in this paper to address an angular velocity tracking objective. 


\section{References}

[1] S. P. Timoshenko, On the transverse vibrations of bars of uniform cross-section, Philosophical Magazine 43 (1922) 125-131.

[2] M. S. D. Queiroz, M. Dawson, S. Nagarkatti, F. Zhang, Lyapunov-Based Control of Mechanical Systems, Birkhauser, Boston, 2000.

[3] J. U. Kim, Y. Renardy, Boundary control of the Timoshenko beam, SIAM Journal of Control and Optimization 25 (1987) 1417-1429.

[4] O. Morgul, Dynamic boundary control of the Timoshenko beam, Automatica 28 (1992) 1255-1260.

[5] C. Mei, Hybrid wave/mode active control of bending vibrations in beams based on the advanced Timoshenko theory, Journal of Sound and Vibration 322 (2009) 29-38.

[6] T. C. Manjunath, B. Bandyopadhyay, Vibration control of Timoshenko smart structures using multirate output feedback based discrete sliding mode control for SISO systems, Journal of Sound and Vibration 326 (2009) 50-74.

[7] G. Xu, H. Wang, Stabilisation of Timoshenko beam system with delay in the boundary control, International Journal of Control 86 (2013) 1165-1178.

[8] W. He, T. Meng, J. K. Liu, H. Qin, Boundary control of a Timoshenko beam system with input dead-zone, International Journal of Control 88 (2015) 1257-1270.

[9] W. He, S. S. Ge, C.Liu, Adaptive boundary control for a class of inhomogeneous Timoshenko beam equations with constraints, IET Control Theory \& Applications 8 (2014) 1285-1292.

[10] T. Endo, F. Matsuno, Y. Jia, Boundary cooperative control by flexible Timoshenko arms, Automatica 81 (2017) 377-389.

[11] M. Krstic, A. A. Siranosian, A. A. Smyshlyaev, Backstepping boundary controllers and observers for the slender Timoshenko beam: Part i design, In 45th IEEE Conference on Decision and Control, San Diego, CA (2006) 39383943.

[12] M. Krstic, A. A. Siranosian, A. A. Smyshlyaev, M. Bement, Backstepping boundary controllers and observers for the slender Timoshenko beam: Part ii stability and simulations, In American Control Conference, Minneapolis, MN (2006) 2412-2417.

[13] M. Krstic, A. Smyshlyaev, Boundary Control of PDEs: A Course on Backstepping Designs, Society for Industrial and Applied Mathematics, Philadelphia, PA, 2008.

[14] K. D. Do, Stochastic boundary control design for Timoshenko beams with large motions, Journal of Sound and Vibration 402 (2017) 164-184.

[15] K. D. Do, J. Pan, Boundary control of transverse motion of marine risers with actuator dynamics, Journal of Sound and Vibration 318 (4-5) (2008) 768-791.

[16] T. L. Nguyen, K. D. Do, J. Pan, Boundary control of two-dimensional marine risers with bending couplings, Journal of Sound and Vibration 332 (16) (2013) 3605-3622.

[17] K. D. Do, Boundary control of elastic systems, Journal of Applied Mathematics and Computing 51 (1) (2016) 315339.

[18] K. D. Do, Stochastic boundary control design for extensible marine risers in three dimensional space, Automatica 77 (2017) 184-197.

[19] K. D. Do, Boundary control design for extensible marine risers in three dimensional space, Journal of Sound and Vibration 388 (2017) 1-19.

[20] W. He, S. S. Ge, Vibration control of a flexible beam with output constraint, IEEE Transactions on Industrial Electronics 62 (2015) 5023-5030.

[21] W. He, C. Sun, S. S. Ge, Top tension control of a flexible marine riser by using integral-barrier Lyapunov function, IEEE/ASME Transactions on Mechatronics 20 (2015) 497-505.

[22] W. He, S. Nie, T. Meng, Y. J. Liu, Modeling and vibration control for a moving beam with application in a drilling riser, IEEE Transactions on Control Systems Technology 25 (2017) 1036-1043.

[23] W. He, T. M. D. Huang, X. Li, Adaptive boundary iterative learning control for an Euler-Bernoulli beam system with input constraint, IEEE Transactions on Neural Networks and Learning Systems, In press, DOI: 10.1109/TNNLS.2017.2673865.

[24] W. He, S. S. Ge, B. Voon, E. How, Y. S. Choo, Dynamics and Control of Mechanical Systems in Offshore Engineering, Springer, London, 2014.

[25] Z. H. Luo, B. Z. Guo, O. Morgul, Stability and stabilization of infinite dimensional systems with applications, Springer-Verlag, 1999.

[26] H. T. Banks, R. C. Smith, Y. Wang, Smart material structures, John Wiley \& Son, Paris, 1996.

[27] K. D. Do, J. Pan, Boundary control of three-dimensional inextensible marine risers, Journal of Sound and Vibration 327 (3-5) (2009) 299-321.

[28] K. D. Do, Global stabilization of three-dimensional flexible marine risers by boundary control, Ocean Systems Engineering 1 (2) (2011) 171-194.

[29] K. D. Do, A. D. Lucey, Boundary stabilization of extensible and unshearable marine risers with large in-plane deflection, Automatica 77 (2017) 279-292.

[30] K. D. Do, Modeling and boundary control of translational and rotational motions of nonlinear slender beams in three-dimensional space, Journal of Sound and Vibration 389 (2017) 1-23. 
[31] K. D. Do, Boundary control of slender beams under deterministic and stochastic loads, Journal of Dynamic Systems, Measurement, and Control, In Press.

[32] L. Evans, Partial Differential Equations, American Mathematical Society, Providence, 2000.

[33] J. B. Kuipers, Quaternions and Rotation Sequences: A Primer with Applications to Orbits, Aerospace and Virtual Reality, Princeton University Press, New Jersey, 2002.

[34] W. C. Orthwein, A nonlinear stress-strain relation, International Journal of Solids and Structures 4 (1968) 371-382.

[35] L. Gawarecki, V. Mandrekar, Stochastic Differential Equations in Infinite Dimensions with Applications to Stochastic Partial Differential Equations, Springer, Berlin, 2011.

[36] P. L. Chow, Stochastic Partial Differential Equations, Chapman \& Hall/CRC, Boca Raton, 2007.

[37] H. Khalil, Nonlinear systems, Prentice Hall, 2002.

[38] K. D. Do, Stochastic boundary control design for Timoshenko beams with large motions, International Journal of Control, In Press.

[39] R. A. Adams, J. J. F. Fournier, Sobolev Spaces, 2nd Edition, Academic Press, Oxford, UK, 2003.

[40] M. Krstic, I. Kanellakopoulos, P. Kokotovic, Nonlinear and adaptive control design, Wiley, New York, 1995.

[41] http://www.mmf.de/manual/ksi80vmane.pdf.

[42] G. D. Smith, Numerical solutions of partial differential equations: Finite difference methods, 3rd Edition, Clarendon Press, Oxford, 1985. 